\title{
Experience from a community-based education program in Burkina Faso: The Tostan program
}

Djingri Ouoba

Zakari Congo

Nafissatou J. Diop

Population Council

Molly Melching

Baya Banza

See next page for additional authors

Follow this and additional works at: https://knowledgecommons.popcouncil.org/departments_sbsr-rh

Part of the Community-Based Research Commons, Demography, Population, and Ecology Commons, Gender and Sexuality Commons, International Public Health Commons, Obstetrics and Gynecology Commons, Public Health Education and Promotion Commons, and the Women's Health Commons How does access to this work benefit you? Let us know!

\section{Recommended Citation}

Ouoba, Djingri, Zakari Congo, Nafissatou J. Diop, Molly Melching, Baya Banza, Georges Guiella, and Inge Baumgarten. 2004. "Experience from a community-based education program in Burkina Faso: The Tostan program," FRONTIERS Final Report. Washington, DC: Population Council. 


\section{Authors}

Djingri Ouoba, Zakari Congo, Nafissatou J. Diop, Molly Melching, Baya Banza, Georges Guiella, and Inge Baumgarten 


\title{
Experience from a Community Based Education Program in Burkina Faso
}

\author{
The Tostan Program
}

Mwangaza Action: Djingri Ouoba, Zakari Congo

Population Council: Nafissatou J. Diop

TOSTAN: Molly Melching

UERD: Baya Banza, Georges Guiella

GTZ (Projet Supra Régional de Lutte contre l'Excision) Inge Baumgarten

September 2004

This study was funded by the U.S. AGENCY FOR INTERNATIONAL DEVELOPMENT (USAID) under the terms of Cooperative Agreement number HRN-A-00-98-00012-00 and Population Subagreement nos. AI00.80A, AI00.81A and AI00.82A, and by the GTZ Supra Regional FGC Project. The opinions expressed herein are those of the authors and do not necessarily reflect the view of USAID. 


\section{Summary}

This study describes a project that tested the feasibility and effectiveness of replicating the village empowerment program (VEP) developed by the Senegalese NGO, TOSTAN, in Burkina Faso. Although one of the ultimate goals of this model is the eradication of female genital cutting (FGC), Tostan has found that an effective means to achieve this is by empowering community members to understand and act on their situation through a broadbased functional educational program. TOSTAN's educational model includes four modules on human rights, problem solving, environmental hygiene and women's health. TOSTAN's hypothesis is that communication of technical information, discussion of human rights issues, and development of strategies for social transformation improve the confidence and selfdetermination of women through participatory educational methods. Mwangaza Action, a Burkinabé NGO affiliated with the Co-operative League of the United Stated of America (CLUSA), adapted and implemented the VEP model in Bazega/Zoundwego province. Although originally developed for empowering women, the program implemented in Burkina Faso also involved men because of their key role in such decisions. Frontiers in Reproductive Health, with funding from USAID and the GTZ Supra Regional Project Against FGC, supported the adaptation, implementation and evaluation of the VEP model in 23 villages in the province of Bazega/Zoundwéogo.

To test the effect of the VEP program, and associated social mobilization process on knowledge, attitudes and behaviors around health, hygiene, rights, and FGC, a pre and postintervention comparison group design was used. Forty-six villages participated in the study, with 23 villages in each of the experimental and control groups. Quantitative data were collected through a baseline and end line survey two years later, as well as during an intermediate survey immediately following completion of the intervention. Several groups of respondents were interviewed individually in the intervention communities: women and men who participated in the VEP sessions; husbands of the women participants; and women and men who did not participate. The purpose of interviewing those that did not participate directly in the educational sessions was to determine the degree to which the information communicated was diffused more widely within the communities. In addition, women were asked about the circumcision status of their girl children aged 0-10 years. In the comparison communities, representative samples of men and women were interviewed. In addition, 192 semi-structured interviews and 42 focus group discussions were conducted with facilitators and key informants within the community.

\section{Findings}

Participants in the educational sessions mostly enjoyed the training program. Over three quarter of participants attended all sessions in the full program; the main reasons for not attending all modules were lack of time, travel, illness, pregnancy, and funerals within the family. Participants declared a preference for the hygiene and health modules over the human rights and problem solving modules.

As a direct effect of the program, the inhabitants of the 23 experimental communities undertook activities to improve environmental hygiene through cleaning the schools and health centers, as well as the areas surrounding sources of water. Healthcare huts were constructed, improvements made to homes, and water sources repaired. In addition, legal documents such as birth certificates, identity cards and family booklets were requested and issued, and 43 couples went through an official civil marriage ceremony. Cases of denouncing forced marriage and of acknowledging discrimination against educating girl 
children were also noted. However, community members regretted that the program did not include literacy classes, nor did it include any income generating activities. Also, the change of facilitators, which took place after the first phase of training, was not appreciated.

In terms of awareness, the VEP contributed to a significant increase in awareness about human rights, women's health, and FGC, both among women and men participating in the program. Men and women who had not attended the classes but who were indirectly exposed to the information communicated in the intervention communities also increased their level of awareness of human rights. Mwangaza encouraged a system of formally expecting participants to share the information learned with other individuals in the community as this had been effective in Senegal, but it did not work well in this context, in that it limited the spread of information throughout the whole of the community.

Levels of awareness on the issues covered were much higher among those who had participated in the program. Women and men participating in the program were better able to mention at least two modern methods of family planning, they accepted that there were advantages in spacing births, and they knew that at least three antenatal consultations should be undertaken. They were also more able to identify at least two danger signs during pregnancy, knew that a person in good health could be a carrier of the HIV virus, could refer to at least two ways of preventing STIs and HIV/AIDS, and could mention at least two dangers of FGC. Overall, men had higher levels of awareness than women, except for antenatal consultations. All improvements could be attributed to the program given that levels of awareness in the comparison communities did not improve or improved less than in the intervention communities.

In terms of attitudes, positive improvements in attitudes were seen among both women and men participating in the program. These improvements included perceptions that sexual discrimination could change, approval of modern contraceptive methods, denunciation of violence against women, disapproval of FGC, and willingness to discuss FGC with other people. An increasing proportion of women whose daughters were cut regretted this and intention to cut daughters in the future decreased significantly among the participant group. There appears to have been little diffusion of these attitudinal changes among those interviewed who did not participate, as there were no significant differences compared with those in the comparison area.

In terms of behavior change, it was noted that the number of violent acts against women in the communities reduced considerably, with a significantly higher decrease in the intervention communities. Concerning eradication of FGC, a transition towards abandonment has already started in this province, as indicated by the small proportion of girls reported to be cut at baseline. In the intervention group overall, this low prevalence of FGC did not change, but the proportion of girls aged 0 to 10 years who had not been cut clearly increased. Indeed, no girls under the age of 5 years were reported to have been cut, compared to three percent in the comparison area. Nevertheless, the baseline survey also showed a lowering of the age of cutting. Conclusions about the impact of this intervention on FGC abandonment are difficult to draw, however, given the context of Burkina Faso where the law forbidding the practice is well known and strongly enforced, and may therefore influence the validity of responses to questions about cutting.

It should be noted that participants in the educational program had a higher level of education (formal schooling, literacy programs and other previous training) than the others, and that 
among the men there was a high proportion of bachelors. In addition, these participants were younger than the rest. They had a high level of mobility (with many outside contacts) and were generally open to change and already in favor of giving up FGC. This skewed selection of participants could have led to the neglect of less active members of the population and those who were more vulnerable to sustaining the practice.

Greater social mobilization against FGC was observed in the intervention communities. By the endline survey, more than 90 percent of peopled interviewed reported having discussed the issue, and more than half of them had been present at the public declaration organized in May 2003. Several opportunities were offered to the women to express themselves in public and in front of the administrative authorities as well as the customary and religious authorities. These opportunities had the effect of convincing the women about the possibility of changing the ways in which women are regarded in the community.

The study also evaluated the combined effect of the education program and the social mobilization process on the community's intention to give up FGC. On the whole, both men and women disapproved of FGC and did not want to cut their daughters. The majority of the men and women who participated in the education program had been present at the public declaration, and most of them held that the decision expressed during the declaration would be followed and that girls would no longer be cut. Even though the intention to share the issues discussed during the education sessions did not work very well, the education program itself aroused interest among those who had been contacted, as well as in the neighboring villages. This interest was demonstrated by the presence of many community members at the public declaration, which could be interpreted as an intention to work against FGC. In the existing social climate among the Burkinabé, where there is widespread awareness of the law forbidding FGC, the education program has contributed to strengthening the existing measures and to a significant improvement in the level of awareness of human rights and reproductive health. 


\section{Remerciements}

Mwangaza action et le Population Council tiennent à adresser leurs remerciements à tous ceux qui ont contribué à la réalisation de cette étude. Une réalisation qui aurait été difficile sans le concours et le soutien de plusieurs partenaires.

Nos remerciements vont à l'endroit de l'USAID et de la GTZ dont le financement aura permis de mettre en place le programme de renforcement des capacités et la réalisation des différentes études d'évaluation. Nous ne saurons oublier l'ONG sénégalaise TOSTAN pour la collaboration fructueuse dans le transfert des compétences et dans la mise en œuvre du programme. Qu'elle y trouve là le fruit de son labeur dans la conception d'un tel programme susceptible de relever le défi de l'éradication de l'excision!

Nos remerciements vont également à l'endroit de l'UERD. Sa rigueur et son professionnalisme tout au long de l'élaboration de l'échantillon, de la formation des enquêteurs, de la collecte, de la saisie et du traitement des données ont permis les résultats du présent travail.

Nous tenons également à remercier le comité national de lutte contre l'excision (CNLPE) qui a pris une part très active aussi bien dans le processus de réflexion, de la finalisation des outils de terrain que dans les suggestions d'amélioration des différents rapports d'étude.

Nous devons aussi remercier les enquêteurs et enquêtrices qui ont contribué de façon significative à la réussite des enquêtes, souvent dans des conditions difficiles. Nous associons à ces remerciements les agents de saisie dont la célérité nous a permis de disposer des fichiers de données dans des temps record.

L'exécution de l'enquête a été facilitée par l'appui et la disponibilité de personnes, de responsables de structures et d'institutions. A ce titre, nos remerciements vont à l'endroit :

- Du comité technique pour les questions et suggestions lors de la diffusion des résultats des opérations d'enquête. Leur attention a grandement contribué à amender et à améliorer les documents partiels.

- Des responsables départementaux qui ont accueilli les différentes équipes sur le terrain et leur ont assuré un cadre adéquat de travail.

- Des responsables ou délégués administratifs villageois qui ont permis de rencontrer les populations et facilité le travail de collecte des données.

- Des populations des aires sanitaires de Béré, Bindé, Sondré dans le Zoundwéogo et celles des aires sanitaires de Ipélcé, Rakaye Mossi, Sambin, Doulougou, Saponé dans le Bazéga. Leur accueil et leur disponibilité à répondre aux questions ont facilité le recueil des informations.

Cette liste n'est certainement pas exhaustive, plusieurs autres personnes ayant apporté une précieuse contribution pour la réalisation de ce travail. Qu'elles trouvent ici l'expression de notre sincère reconnaissance! 


\section{Résumé}

Cette étude documente la réplication d'un programme de renforcement des capacités au Burkina Faso. Ce programme conçu par TOSTAN, une ONG sénégalaise utilise une approche intégrée en abordant plusieurs aspects relatifs à la situation des femmes, associés à une mobilisation communautaire pour une déclaration publique en faveur de comportements plus positifs. Sa stratégie s'articule autour de trois fronts : éducation de base, débats publics et déclarations publiques. Le programme d'éducation comporte 4 modules : Hygiène, Droits Humains, résolution des problèmes et santé de la femme. L'hypothèse de TOSTAN est que la communication d'information technique, les discussions sur les questions de droits humains, et le développement de stratégies pour la transformation sociale améliore la confiance et la détermination des femmes. L'ONG Mwangaza Action a mis en œuvre ce programme dans la province du Bazéga/Zoundwégo. Conçu au départ pour les femmes, le programme a également impliqué les hommes, aspect d'autant plus important que les pères sont ceux qui prennent les décisions, notamment en ce qui concerne l'excision des filles (UERD, 1997). Frontières en Santé de la reproduction avec un financement de l'USAID et le Projet Supra Régional de Lutte contre l'Excision exécuté par la GTZ ont financé l'adaptation, la mise en œuvre du programme et son évaluation dans 23 villages de la province du Bazéga/Zoundwéogo.

Pour tester les effets du programme et des activités de mobilisation sociale sur les connaissances, attitudes et comportements en matière de santé, d'hygiène de droits et d'excision, la méthode population type-population témoin a été utilisée. Quarante-six villages ont participé à l'étude, dont 23 villages devant accueillir le programme et 23 villages servant de comparaison. Les données quantitatives ont été collectées avant le démarrage du programme, immédiatement après le programme et une année plus tard. Cinq groupes ont été interviewés individuellement dans les zones d'intervention : les femmes et les hommes participants au programme ; les maris des femmes participantes ; et les femmes et les hommes ne participant pas au programme mais vivant dans le village. Ce dernier groupe a été interviewé pour voir l'effet de diffusion de l'information dans la communauté. Le statut d'excision des filles de 0 à 10 ans de toutes les femmes interrogées a aussi été saisi. Pour comparer les potentielles autres influences, des couples ont été interrogés dans une zone de comparaison ne devant recevoir aucune intervention, avant le démarrage du programme et une année après sa mise en œuvre. Des interviews semi-structurées (192) et des focus group (42) ont été menés avec les facilitateurs et des informateurs clés de la communauté.

\section{Résultats}

En général, les participants des 23 villages de la zone expérimentale ont beaucoup apprécié le programme d'éducation. Plus des $3 / 5^{\text {ème }}$ des participants ont suivi toutes les séances ; Les principales raisons d'absence sont le manque de temps, les voyages, la maladie, les grossesses et les funérailles. Les participants ont déclaré avoir préféré les modules sur l'hygiène et la santé à ceux sur les droits humains et la résolution des problèmes.

L'effet direct du programme a été la mobilisation sociale des 23 communautés autour de l'hygiène, de la santé et des comportements citoyens. Les écoles, les centres de santé et l'environnement des sources d'eau ont fait l'objet de campagne d'assainissement. Des cases de santé ont été construites, les pompes à eau réparées, des certificats de naissance et des cartes d'identité délivrées. Les cas de mariage forcés et de discrimination envers la scolarisation des filles ont été dénoncés. Cependant les communautés regrettent que le 
programme n'ait pas inclus d'alphabétisation ou d'activités génératrices de revenus. Les changements de facilitateurs, après la première phase du programme, n'ont pas été appréciés par les communautés.

En terme de connaissance, le programme a contribué à améliorer significativement les connaissances sur les droits humains, le planning familial, les consultations prénatales, les signes de danger de la grossesse, l'excision, les IST/VIH/SIDA, les différentes formes de violences contre les femmes et les formes de discrimination contre les femmes pour les 2 groupes ayant participé au programme. Les femmes et les hommes qui n'ont pas participé au programme, mais qui vivent dans les villages d'intervention, ont aussi augmenté leurs connaissances sur les droits humains. Le système de partage de l'information que Mwangaza a encouragé n'a cependant pas fonctionné pleinement dans le contexte Burkinabé, tel qu'il le fait au Sénégal. La diffusion de l'information dans toute la communauté a eu un effet limité.

Les niveaux de connaissance sont beaucoup plus élevés chez les femmes et les hommes participants au programme. Ils étaient plus capables de citer au moins deux méthodes modernes de contraception, de reconnaître les avantages de l'espacement des naissances, et de savoir qu'il faut 3 visites prénatales durant la grossesse. Ils étaient aussi capables d'identifier au moins deux signes de danger durant la grossesse, deux dangers de l'excision, savoir qu'une personne saine peut être porteuse du VIH et connaître les moyens de prévention des IST. En général, les hommes ont des niveaux plus élevés de connaissance que les femmes, sauf pour les consultations prénatales. Toutes ces améliorations peuvent être attribuées au programme dans la mesure où les niveaux de la zone de comparaison n'atteignent pas ceux des groupes d'intervention.

En terme d'attitude, des améliorations positives ont été observées parmi les femmes et les hommes participants au programme. Ces améliorations touchent les perceptions sur les discriminations, l'approbation de la contraception moderne, la dénonciation des violences envers les femmes, la désapprobation de l'excision et l'envie de discuter de l'excision. Concernant l'excision, plus d'hommes et de femmes participants déclarent que la pratique n'est plus nécessaire et les femmes sont plus nombreuses à regretter l'excision de leurs filles et à ne pas approuver la pratique. Elles soutiennent presque toutes que leurs filles ne seront plus excisées. Il n'y a cependant eu qu'une faible diffusion de ces nouvelles attitudes parmi la population ne participant pas directement au programme, puisque les niveaux atteints ne sont pas différents de ceux de la zone de comparaison.

En terme de changement de comportement, il a été noté que le nombre d'actes de violence a diminué dans la communauté, avec une baisse significative pour les groupes d'intervention. Concernant l'excision, une transition vers l'abandon de la pratique a déjà commencé dans cette province. Les données de l'étude de base montraient déjà de faibles proportions de filles excisées. Dans le groupe d'intervention, la proportion des filles de 0-10 ans non excisées a augmenté. En plus aucune fille de moins de 5 ans n'a été déclarée excisée. Les données de base montrent aussi une baisse de l'âge à l'excision. Les conclusions sur l'impact de l'intervention sur l'excision sont difficiles à tirer dans le contexte du Burkina où la loi interdisant la pratique est très connue et fortement appliquée. Ceci peut influencer la validité des réponses.

Il faut noter que les participants au programme avaient un niveau d'instruction plus élevé (instruction scolaire, alphabétisation et autre formation antérieure) que les autres, avec chez les hommes une proportion élevée de célibataires. De plus, les participants sont plus jeunes 
que les autres. Ces jeunes personnes bénéficient d'une grande mobilité (avec de nombreux contacts extérieurs) et sont d'une façon générale aptes au changement et déjà favorables à l'abandon de l'excision. Ce biais de sélection peut conduire à négliger les « sédentaires » et les plus vulnérables à soutenir le changement.

Une forte mobilisation sociale sur la question de l'excision a été observée dans les communautés d'intervention. Lors de l'évaluation finale, plus de $90 \%$ des personnes ont discuté de la question, et plus de la moitié étaient présentes à la déclaration publique organisée en mai 2003. Plusieurs opportunités ont été offertes aux femmes de s'exprimer en public devant les autorités administratives, coutumières et religieuses. Ceci a permis aux femmes d'être convaincues qu'un changement de leur statut est possible.

L'étude a aussi évalué l'effet combiné du programme d'éducation et de la mobilisation sociale sur la volonté des communautés d'abandonner l'excision. De plus en plus d'hommes et de femmes affirment être prêts pour discuter en public de la question, une tendance présente également parmi les femmes de la zone de comparaison, mais avec un degré moindre. Les populations de la zone d'intervention demeurent convaincues que la déclaration publique sera respectée. Mais pour cela, elles préconisent de sensibiliser davantage les leaders de la communauté afin de toucher toute la population entière des villages. Malgré le disfonctionnement du système de parrainage, le programme d'éducation a suscité un intérêt auprès des filleuls et des interlocuteurs des participants mais également dans les villages voisins de la zone d'intervention. Un intérêt qui s'est manifesté par la présence de nombreux habitants de ces villages à la cérémonie de déclaration publique. Ce qui peut être interprété comme une intention de lutter contre la pratique de l'excision. Dans un climat social burkinabé marqué par la connaissance générale de la loi interdisant la pratique de l'excision, le programme d'éducation aura contribué à renforcer les mesures existantes et à améliorer significativement des connaissances en matière de droits humains, d'hygiène et de santé de la reproduction. 


\section{T A B L E D E S M A T I E R E S}

\section{Summary}

Remerciements

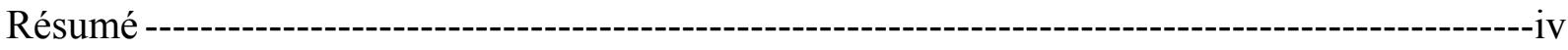

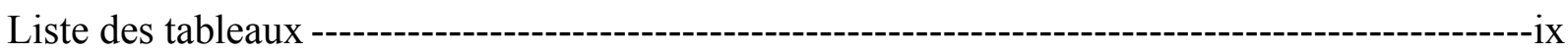

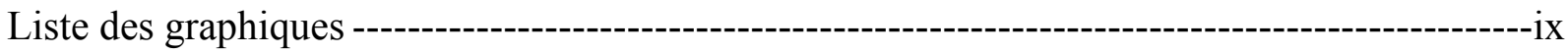

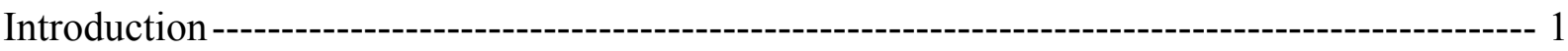

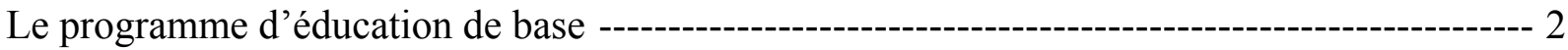

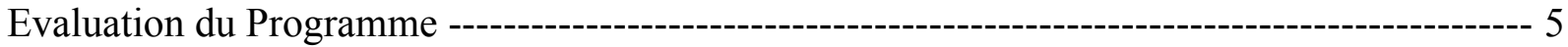

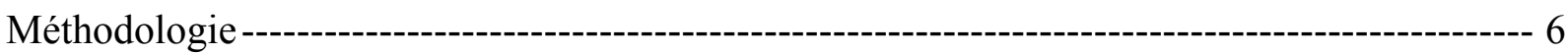

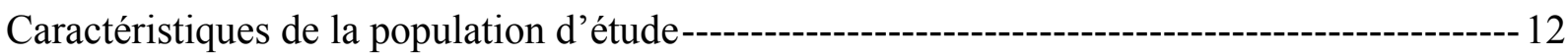

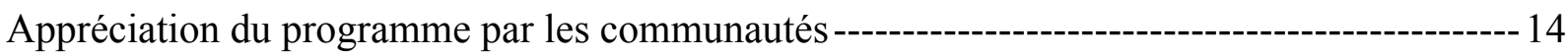

Contribution du VEP sur les Connaissances, Attitudes et Comportements ----------------------- 18

Les Droits Humains ----------------------------------------------------------------------------- 18

Les violences faites aux femmes ----------------------------------------------------------- 22

Connaissance sur la santé et Utilisation des services ------------------------------------- 24

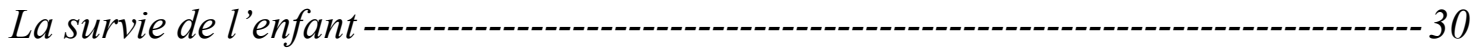

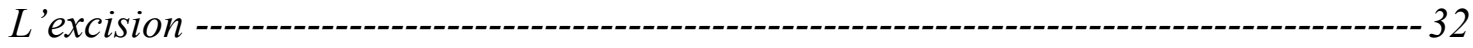

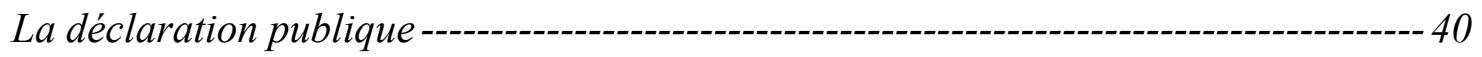

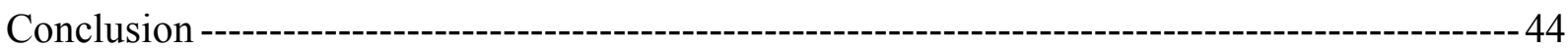

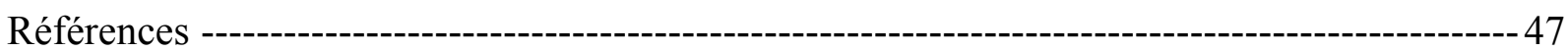

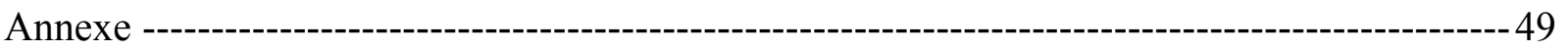




\section{Liste des tableaux}

Tableau 1 : Répartition des différents groupes de comparaison ------------------------------------------------------------- 7

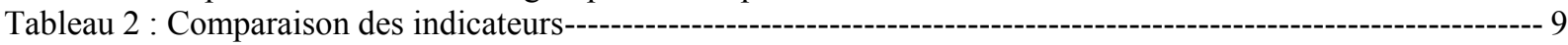

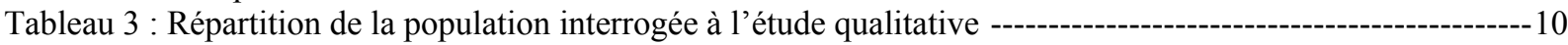

Tableau 4 : La population d'étude selon les différentes periodes de collecte------------------------------------------11

Tableau 5 : Régularité (en \%) de la participation des enquêtés lors des différentes collectes d'information-------12

Tableau 6 : Répartition des participants suivant leurs attentes avant la formation -------------------------------16

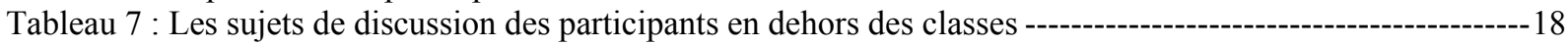

Tableau 8 : Connaissances des conjoints_des femmes participantes sur les droits humains ------------------------19

Tableau 9 : Niveau de connaissance et attitudes des hommes et des femmes sur les droits humains----------------20

Tableau 10 : Connaissance et attitude des hommes et des femmes face aux violences faites aux femmes ---------23

Tableau 11 : Connaissance et attitude des hommes et des femmes sur la santé de la reproduction---------------26

Tableau 12 : Connaissances des conjoints_des femmes participantes sur la PF et la santé------------------------27

Tableau 13 : Niveau de connaisssance et attitudes des hommes et des femmes sur les IST/ VIH/SIDA --------31

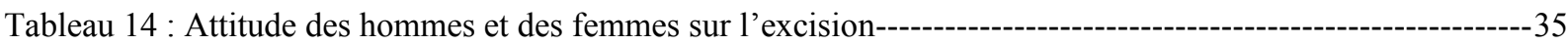

Tableau 15 : Connaissances et Attitude des conjoints des femmes participantes sur l'excision --------------------36

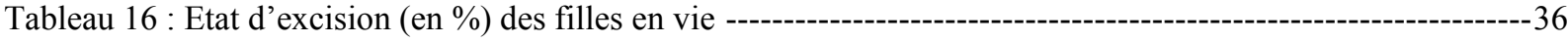

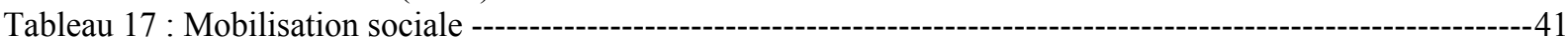

Tableau annexe 1 : Répartition (en \%) des femmes des différentes zones d'enquête selon leur participation au

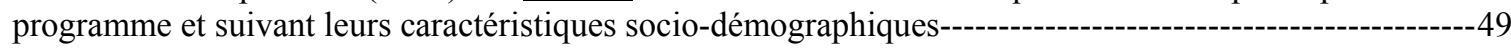

Tableau annexe 2 : Répartition (en \%) des hommes des différentes zones d'enquête selon leur participation au programme et suivant leurs caractéristiques socio-démographiques--------------------------------50

Tableau annexe 3 : Caractéristiques socio-démographiques des conjoints de femmes participantes au programme51

Tableau annexe 4 : Proportion cumulée des filles non excisées ---

\section{Liste des graphiques}

Graphique 1 : Proportion des participants suivant les modules qu'ils jugent intéressants ----------------------------15

Graphique 2 : Les apports du programme selon les participants -----------------------------------------------------16

Graphique 3 : Les interlocuteurs des participants sur les modules du programme-----------------------------------17

Graphique 4 : A entendu parler des droits humains --------------------------------------------------------------------19

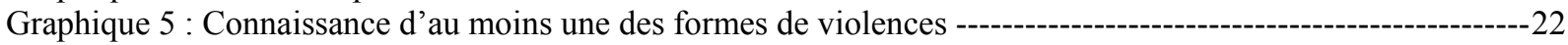

Graphique 6 : Identification en zone expérimentale des changements intervenus au niveau communautaire

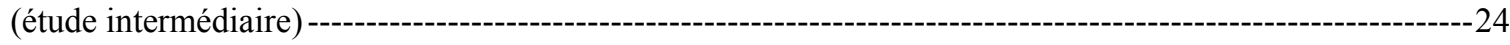

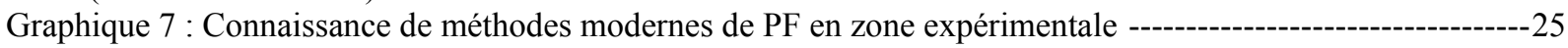

Graphique 8 : Désir d'utilisation future d'une méthode contraceptive ---

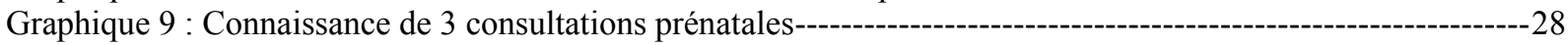

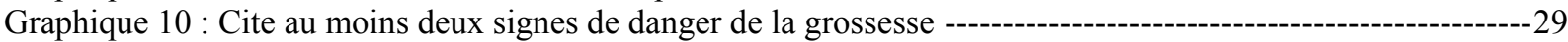

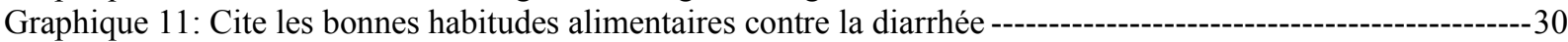

Graphique 12 : L'excision n'est pas une pratique nécessaire -----

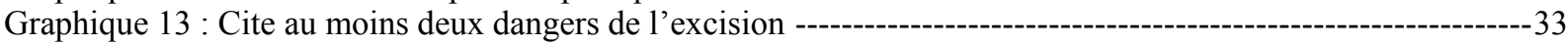

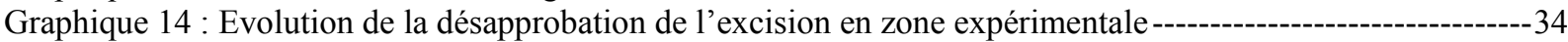

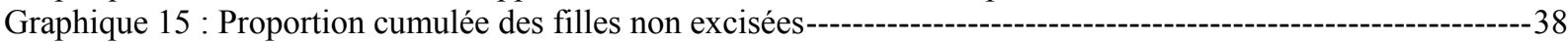

Graphique 16 : Discute de l'excision avec les autres -------------------------------------------------------------------- 39

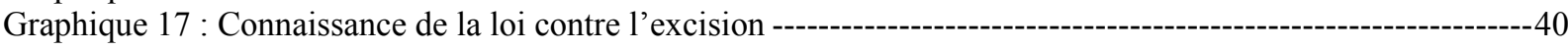

Graphique 18 : Informations sur la cérémonie de déclaration publique ----------------------------------------------42 


\section{Introduction}

La pratique de l'excision reste encore une réalité au Burkina Faso où elle touche $72 \%{ }^{1}$ des femmes âgées de 15 à 49 ans (EDSBF-II, 1998/99). La mise en place d'un Comité National de Lutte contre la Pratique de l'Excision (CNLPE) en 1990 traduit manifestement la volonté des autorités administratives et politiques à éradiquer cette pratique. La souscription à cette lutte s'est traduite sur le plan juridique par l'adoption en 1996 de la loi interdisant, sous peine de punition, l'excision.

A l'origine, la pratique de l'excision faisait partie d'un ensemble de coutumes servant pour les jeunes filles de rite de passage à l'âge adulte. Elle avait également pour rôle de réduire la libido et garantir ainsi la fidélité conjugale de la femme, de maintenir la coutume. Elle s'est ainsi ancrée dans des valeurs sociales, croyances et normes culturelles et reste très liée à des idées fortes sur l'identité, la sexualité, le genre, les droits de l'homme et le pouvoir (Izett et Toubia, 1999).

Dans le but d'éradiquer la pratique de l'excision, plusieurs approches ont été utilisées. L'une des approches est le programme d'éducation conçu par TOSTAN, une ONG sénégalaise. Ce programme utilise une approche intégrée en abordant plusieurs aspects relatifs à la situation des femmes, associé à une mobilisation communautaire pour l'amener à faire une déclaration publique en faveur de comportements plus positifs. Cette approche semble être prometteuse et pérenne pour la réduction de la pratique puisque la stratégie s'articule autour de trois fronts : éducation de base, débats publics et déclarations publiques. De plus, ce programme soutient les stratégies issues du PLA (Approche de la formation participative) qui indique que l'abandon de la pratique de l'excision passe par l'éducation de la population et par l'implication de l'ensemble de la communauté dans l'organisation des activités (LSC, 1998). Conçu au départ pour les femmes, le programme a également impliqué les hommes, aspect d'autant plus important que dans la zone d'intervention choisie, les pères sont ceux qui prennent surtout les décisions, notamment en ce qui concerne l'excision des filles (UERD, 1997).

Afin de tester cette nouvelle stratégie, la zone du laboratoire de santé communautaire (LSC) dans les provinces du Bazèga et du Zoundwéogo a été retenue. Ce laboratoire a été mis en place en 1996 par le Ministère de la Santé et des études régulières ont permis de fournir une bonne connaissance sociologique, culturelle et sanitaire des populations de cette zone.

Dans cette zone du LSC, on notait en 1996, une prédominance de l'ethnie mossi (97\%) avec $92 \%$ de femmes et $92 \%$ d'hommes n'ayant aucun niveau d'instruction. Les catholiques et les musulmans représentaient respectivement $39 \%$ et $38 \%$. Parmi les femmes en âge de procréer, $90 \%$ d'entre elles sont excisées. Cinquante et un pour cent des femmes et $41 \%$ des hommes ont déclaré approuver la pratique de l'excision. En 1998, l'indice synthétique de fécondité y était de 6,4 enfants par femme avec un taux de mortalité infanto-juvenile de 157\%o. Parmi les femmes ayant déjà eu au moins une grossesse, $48 \%$ ont effectué entre 2 et 3 visites prénatales lors de la dernière grossesse. L'âge moyen au premier mariage est de 17 ans et $59 \%$ des

\footnotetext{
${ }^{1}$ L'enquête nationale sur l'excision de 1996 donne une prévalence de $66 \%$ chez les femmes âgées de 15 ans et plus. L'objet d'étude, la population cible et les méthodologies différentes expliquent cette différence entre les deux proportions. Cela ne saurait signifier une augmentation de la pratique.
} 
femmes vivent dans un foyer polygame. Les femmes mariées utilisant une méthode contraceptive moderne représentaient $5,4 \%$ contre $7 \%$ pour les méthodes traditionnelles.

\section{Le programme d'éducation de base ${ }^{1}$}

La mise en place du programme d'intervention a nécessité le développement d'une démarche de mobilisation sociale. Cette démarche s'articule autour de cinq phases : la phase préparatoire, la phase d'orientation, la phase d'organisation, la phase de mise en œuvre et la phase d'évaluation et de dissémination.

La phase préparatoire a consisté dans un premier temps à la traduction en Mooré ${ }^{2}, \mathrm{du}$ curriculum de TOSTAN $^{3}$ et à la formation de l'équipe d'encadrement. Cette équipe d'encadrement, composée de 2 chargés de programme de Mwangaza et de 3 superviseurs a effectué un séjour de un mois à Thiès (au Sénégal) pour une formation par l'équipe de l'ONG TOSTAN. La participation de cette équipe à la déclaration publique de promotion de la femme de Aéré Lao et de Karcia a permis à ces responsables de s'imprégner du processus organisationnel et d'animation d'une telle cérémonie.

La phase d'orientation est articulée autour du recrutement des facilitateurs communautaires et de l'organisation des rencontres d'orientation et d'information des facilitateurs sur les grandes lignes du Programme. Contrairement au Sénégal ${ }^{4}$ où les facilitateurs émanent de la communauté, les facilitateurs communautaires du Burkina ont été recrutés en ville. Un stage d'immersion de 3 semaines a été ensuite effectué dans la zone d'intervention pour permettre à ces facilitateurs de faire connaissance avec leur futur lieu de travail. Des rencontres informelles ainsi que des assemblées générales de présentation et discussion des grandes lignes du programme ont eu lieu avec les autorités administratives et techniques ainsi que les responsables locaux et les représentants des villages. Spécifiquement dans chaque village, le programme a été présenté à l'ensemble de la communauté.

La phase d'organisation a consisté à mettre en place les comités villageois de gestion et à définir le rôle et la responsabilité des membres. Avec ces comités, les critères d'identification et du choix de 30 femmes et de 30 hommes dans chaque village ont été définis et mis en œuvre.

La phase de mise en æuvre du programme est la plus importante du processus. Elle a consisté à former les facilitateurs sur le contenu pédagogique, à démarrer les classes pour femmes puis les classes pour hommes dans chaque village.

La langue de travail et de communication étant le mooré, un séminaire d'une semaine a été organisé pour leur permettre de mieux maîtriser les règles de transcription en mooré. Ensuite, un séminaire de formation de trois semaines sur le paquet pédagogique a été organisé pour leur permettre une plus grande familiarité avec cet outil de travail indispensable à toute transmission de connaissances. Ce séminaire a porté sur les modules suivants : l'éducation

\footnotetext{
${ }^{1}$ Les informations de cette section sont essentiellement tirées du document synthétique du processus de mise en œuvre du Programme TOSTAN au Burkina Faso, Avril 2003, 45 p.

${ }^{2}$ Le mooré est la langue parlée par les mossi, ethnie majoritaire du pays mais également celle de la zone d'intervention.

${ }^{3}$ Les modules ont été développés au Sénégal et étaient rédigés en français.

${ }^{4}$ L'ONG TOSTAN a une longue expérience de travail dans les zones d'intervention avec surtout ses programmes consacrés aux adultes.

C'est ce qui lui a permis d'identifier et de former des personnes locales ayant des compétences nécessaires pour cette formation.
} 
aux droits humains, le processus de résolution des problèmes, les techniques de conduite des séances, la technique d'utilisation de la boîte à image, la pédagogie des adultes, les techniques de tenue et d'animation des séances dans les classes. Durant la seconde campagne du programme, un deuxième séminaire de formation de trois semaines a été organisé. Celuici a porté sur les thèmes suivants : la santé de la femme, les violences faites aux femmes, l'hygiène et la prévention des maladies. On y ajoutera des animations sur des sujets tels que la maternité à moindre risque, la politique nationale en matière de vaccination et l'approche genre.

Un facilitateur a été affecté dans chacun des 23 villages et chaque village avait la responsabilité de loger son facilitateur communautaire. Ainsi l'équipe des 23 facilitateurs et leurs 3 superviseurs ont séjourné dans les villages durant tout le temps du programme. Ils partageaient la vie quotidienne des villageois et avaient avec eux, beaucoup plus de contact, de sorte à instaurer un climat de confiance, nécessaire aux multiples sollicitations du programme.

Après la session de 2 jours qui a permis de former les membres des comités villageois de gestion sur les techniques de gestion des réunions, le programme a doté chaque village de matériels et fournitures composés essentiellement de bancs, de corde et pinces à linge, de boîtes à images, de cahiers et de crayons.

Une fois sur le terrain, c'est avec les participants et les participantes que les modalités pratiques de démarrage du programme (jours et horaires des séances) ainsi que la date de début des séances ont été définies. De façon générale, les séances d'animation ont eu lieu tous les deux jours, d'une durée par séance de 2 heures. Quatre modules étaient prévus au programme. Compte tenu du calendrier saisonnier des pluies, les 63 séances d'animation ont été dispensées aux classes des hommes et à celles des femmes au cours de deux périodes différentes. La première a eu lieu de février à mai 2001 et la seconde de décembre 2001 à mars 2002, soit une durée effective de formation de 8 mois. La formation qui a eu lieu au cours de la première période a été consacrée aux deux premiers modules à savoir l'Education aux droits humains et le Processus de résolution des problèmes. Les classes des hommes ayant démarré un mois après celles des femmes, le module sur le Processus de résolution des problèmes ne leur a pas été enseigné. Celle de la seconde période s'est appesantie sur les autres modules que sont l'hygiène et la prévention ainsi que la santé de la femme. Avant l'enseignement des deux derniers modules, un rappel de l'essentiel des premiers modules a constitué le fond des premières séances lors de la seconde période.

Concomitamment aux séances dans les classes, les facilitateurs ont animé des séances publiques à l'intention des autres villageois mais également dans certains villages non couverts par le programme.

Afin de favoriser la diffusion de l'information dispensée au cours des séances, le programme d'éducation a initié le principe du parrainage qui consiste pour chaque participant à se choisir un filleul (ou une filleule) du village à qui il fait partager ses connaissances acquises.

La dernière phase est celle de l'évaluation et de la dissémination. Elle a consisté à effectuer le suivi des activités, à apporter des solutions aux difficultés de mise en œuvre et à diffuser l'expérience acquise. A la fin de chaque séance de formation, les participants sont sollicités pour évaluer la qualité de l'enseignement du jour. Trois superviseurs étaient chargés du suivi 
des activités des facilitateurs tandis qu'au niveau du village ce suivi est assuré par le comité villageois de gestion.

Dans chacun des 23 villages, une réunion a regroupé toutes les personnes et structures villageoises impliquées dans le programme ainsi que les autres membres de la communauté. Ces réunions ont permis de faire le point de l'exécution du programme au niveau du village. Ces réunions villageoises ont également permis de préparer les forums inter-villages par aire de santé qui à leur tour ont permis aux délégations villageoises d'échanger sur les réalisations, les acquis, les forces et les faiblesses du programme. La même opération de réunion a également eu lieu à la fin de la seconde période de formation.

A la fin du programme, une cérémonie de déclaration publique d'abandon de l'excision a eu lieu à Béré, chef lieu du dit département le 03 mai 2003. Cette cérémonie a regroupé les participants et participantes des 23 villages couverts par le programme, mais aussi beaucoup d'autres personnes venues des villages environnants. On peut noter également la présence effective des chefs coutumiers et religieux de la zone. Elle a surtout été marquée par la déclaration faite par une femme au nom de toutes les communautés et le geste rituel du représentant des chefs coutumiers qui valide l'engagement pris d'abandonner la pratique de l'excision.

\section{Quelques acquis du programme}

\section{Au niveau communautaire :}

Durant le déroulement du programme, des actions communautaires multiples ont été observées. Elles vont de la préservation de l'environnement à l'amélioration du cadre de vie, en passant par les actes civiques ainsi que les actions d'hygiène publique.

Le premier effet vise à améliorer l'hygiène du village. Les écoles, les centres de santé et de promotion sociale (CSPS), les alentours des points d'eau ont été nettoyés dans la plupart des villages. Dans l'ensemble des 23 villages, des cases de santé ont été construites pour remettre en activité les accoucheuses villageoises formées et/ou recyclées. On notera également la confection de foyers améliorés par les femmes et leurs regroupements pour mener des activités génératrices de revenu. A Béré par exemple, un nouveau comité de point d'eau fut installé et la pompe manuelle du marché, en panne depuis 3 ans, fut réparée. Certains actes civiques ont été enregistrés tels que l'établissement de jugements supplétifs d'acte de naissance, de carte d'identité et de livret de famille. Enfin, près de 300 personnes ont assisté à une cérémonie officielle où 43 couples ont scellé un mariage civil. On a pu noter également un cas de dénonciation de mariage forcé et la constatation d'un cas de discrimination par rapport à l'éducation des enfants.

Il a également été noté une prise de conscience locale des dangers de l'excision au regard des sketchs organisés par les villageois et exécutés par les femmes elles même. Et le plan d'action élaboré par les représentants des villages identifie l'excision comme une violence faite aux femmes et préconise la sensibilisation de la population sur les dangers de cette pratique.

Les actions observées ne sont pas toujours quantifiables mais elles manifestent clairement le désir des populations à s'investir davantage dans la résolution de leurs problèmes. 
Au niveau des facilitateurs ${ }^{1}$

De niveau scolaire supérieur ou égal au bac, les facilitateurs ont résidé dans les villages, partageant pour la plupart la maison et le repas avec les populations rurales. Cette expérience a permis à ceux-ci de mieux s'adapter à leur milieu de travail et d'instaurer un climat de confiance et une meilleure communication avec les participants du programme. Dans la majeure partie des cas, la cohabitation a été très fructueuse et appréciée par les populations.

Cependant, les facilitateurs ont fait remarquer que la durée de leur formation est restée assez courte et qu'il aurait fallu mettre l'accent dans les villages sur le cadre d'accueil et de formation des participants. Ils auraient souhaité pouvoir disposer de brochures récapitulatives des principaux axes de la formation leur permettant ainsi une meilleure maîtrise des séances de formation sur le terrain, quelques amélioration du matériel de formation (grandeur des images), une meilleure supervision et un meilleur suivi des activités d'intervention. Ils suggèrent enfin que le contenu des thèmes dispensés soit un peu moins centré sur les femmes et que le processus de recrutement des participants connaisse une amélioration au regard des biais de sélection introduits par les populations elles-mêmes dans l'établissement des listes des participants au programme.

\section{Evaluation du Programme}

\section{Objectifs de l'étude}

L'objectif de l'étude est de fournir aux responsables de programme et à leurs partenaires les effets et l'impact du programme de renforcement des capacités villageoises développé par TOSTAN et répliqué au Burkina Faso.

De façon spécifique, il s'agit de :

- Evaluer les effets du programme de renforcement des capacités sur les niveaux de connaissance, croyance et attitude des femmes et des hommes relatifs aux problèmes de santé de la reproduction et des droits humains ;

- Evaluer les effets du programme de renforcement des capacités sur une meilleure prise de conscience des hommes et des femmes des conséquences négatives de l'excision ;

- Déterminer les facteurs qui favorisent l'implication et la mobilisation des populations en faveur d'un changement social ;

- Evaluer les effets combinés du programme d'éducation et du processus de mobilisation sociale sur la volonté des communautés à abandonner l'excision.

\section{Hypothèses}

Les hypothèses de l'étude s'articulent comme suit :

- Les femmes et les hommes qui ont participé au programme d'éducation auront un niveau de connaissance plus élevé et des attitudes plus positives sur les questions relatives à la santé de la reproduction (y compris l'excision) et aux droits humains, que celles n'ayant pas participé au programme.

\footnotetext{
${ }^{1}$ Cf. Rapport qualitatif auprès des facilitateurs communautaires.
} 
- Les partenaires des femmes ayant suivi le programme d'éducation auront un niveau de connaissance sur les conséquences négatives de l'excision plus élevé, et seront plus en faveur de l'abandon de l'excision.

- Les femmes et les hommes des villages où le programme de renforcement des capacités a été exécuté auront un niveau de connaissance plus élevé des conséquences négatives de l'excision, et seront plus motivés à faire une déclaration publique d'abandon de l'excision que les femmes et les hommes des villages où le programme n'a pas été exécuté.

- Les femmes et les hommes des villages où le programme de renforcement des capacités a été exécuté, et dans lesquels un processus de mobilisation a été mené, auront plus tendance à abandonner l'excision.

\section{Méthodologie}

Procédé de recherche

L'étude utilise un schéma d'évaluation pré et post intervention avec population témoin. Deux zones d'études sont utilisées pour mener des enquêtes sur l'efficacité des interventions, en procédant à des comparaisons entre la zone d'intervention et la zone de comparaison. La zone expérimentale bénéficie du programme éducatif qui devrait concerner 30 femmes et 30 hommes dans chacun des 23 villages de la zone. La zone de comparaison ne bénéficie d'aucune intervention de ce type. Les incidences liées aux aspects temporels sont également évaluées. Il s'agit pour cette présente étude de mesurer les effets réels de la mise en œuvre du programme. Le schéma suivant illustre la procédure de recherche.

Le processus est ainsi libellé :

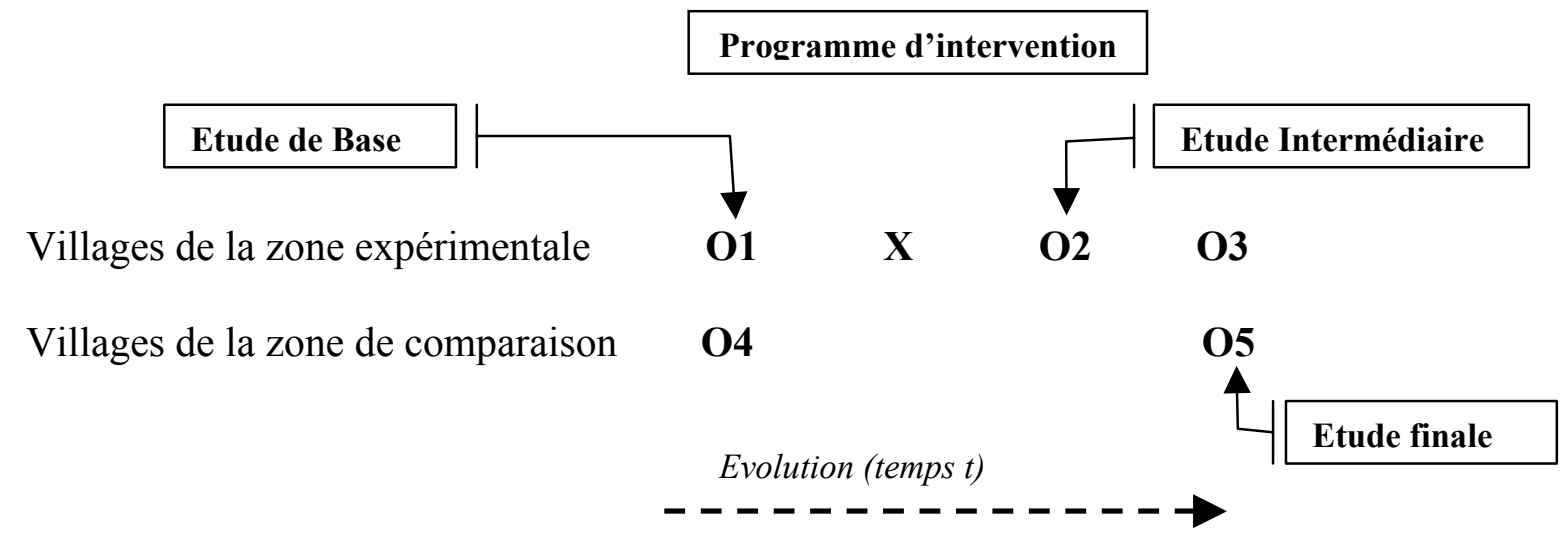

Mesure des connaissances sur la SR, droits humains et attitudes des hommes et $\begin{aligned} \text { O1, } \mathbf{O 4}= & \text { des femmes envers l'excision; } \\ & \text { Proportion des filles de } 0-10 \text { ans non excisées ; }\end{aligned}$

$\mathbf{X}=$ Programme d'éducation et processus de mobilisation sociale ;

O2 = Mesure des connaissances sur la SR, droits humains et attitudes envers l'excision $=$ des femmes;

Mesure des connaissances sur la SR, droits humains et attitudes des hommes et

O3, $\mathbf{O 5}=$ des femmes envers l'excision;

Proportion des filles de 0-10 ans non excisées. 


\section{Les zones d'étude}

Les deux zones d'études font partie des quatre zones que comptait le Laboratoire de Santé Communautaire (LSC), une station de recherche dans la province rurale de Bazèga ${ }^{1}$. Dans le cadre de son programme de santé reproductive appelé programme DBC (Distribution à Base Communautaire), le laboratoire intervenait dans deux zones $(\mathrm{A}, \mathrm{B})$ tandis que l'ONG Save The Children occupait la troisième zone (D). La zone du Zoundwéogo $(C)$ était sans intervention. Elle constituait la zone de comparaison. Aujourd'hui dans le cadre du programme de renforcement des capacités, celle du Zoundwéogo bénéficie de l'intervention tandis qu'une des zones du LSC (D) constitue la zone de comparaison.

La présente zone d'intervention qui est située dans la province du Zoundwéogo regroupe trois aires sanitaires que sont Béré, Bindé et Sondré ${ }^{2}$. Ces aires sanitaires sont regroupées dans deux départements (Bindé et Béré). La zone de comparaison qui est située dans la province du Bazèga quant à elle est constituée de 3 départements (Ipélcé, Saponé et Doulougou) qui regroupent 5 aires sanitaires : Ipélcé, Rakaye Mossi, Sambin, Doulougou et Saponé. Les deux zones sont assez distantes et sont opposées sur le plan géographique.

Dans la zone expérimentale, les 23 villages ont été identifiés sur la base de leur appartenance à l'aire de santé et pour la participation de membres de chaque village dans le comité de gestion des formations sanitaires périphériques, permettant ainsi une meilleure circulation de l'information entre les villages et une meilleure mobilisation des ressources humaines. En zone de comparaison, les 23 villages qui constituent la zone D du LSC ont tous été sélectionnés. Au total, 46 villages sont concernés par l'étude.

\section{Procédures d'échantillonnage}

Il existe quatre unités d'échantillonnage pour lesquelles les données sont collectées : les femmes, les hommes, l'ensemble de la communauté du village et les facilitateurs. Le programme de l'ONG TOSTAN avait été conçu pour les femmes. Mais avec les résultats de recherche montrant la place du père, des classes d'hommes ont aussi été créées. Le tableau 1 présente les six groupes en question :

\section{Tableau 1 : Répartition des différents groupes de comparaison}

\begin{tabular}{|c|c|c|c|}
\hline$A$ & Zone expérimentale & & \\
\hline & $\begin{array}{ll}\text { Femmes } \\
\end{array}$ & \multirow[b]{2}{*}{4} & $\begin{array}{l}\text { Hommes } \\
\end{array}$ \\
\hline 1 & $\begin{array}{l}\text { Les femmes directement exposées à l'intervention, } \\
\text { c'est à dire les bénéficiaires du programme } \\
\text { d'éducation = participantes }\end{array}$ & & $\begin{array}{l}\text { Les hommes directement exposés à l'intervention, } \\
\text { bénéficiant directement du programme d'éducation = } \\
\text { participants }\end{array}$ \\
\hline 2 & $\begin{array}{l}\text { Les femmes indirectement exposées à l'intervention; } \\
\text { ce sont celles vivant dans le village où le programme } \\
\text { d'éducation a été mis en oeuvre mais qui ne sont pas } \\
\text { inscrites dans le programme = non-participantes }\end{array}$ & 5 & $\begin{array}{l}\text { Les hommes indirectement exposés à l'intervention, } \\
\text { partenaires des femmes du village où le programme } \\
\text { d'éducation a été mis en œuvre = non-participants }\end{array}$ \\
\hline \multicolumn{4}{|c|}{ Zone de comparaison } \\
\hline \multirow[b]{2}{*}{3} & Femmes & & $\begin{array}{l}\text { Hommes } \\
\end{array}$ \\
\hline & $\begin{array}{l}\text { Les femmes qui ne sont pas exposées à l'intervention ; } \\
\text { ce sont celles vivant dans les villages de comparaison } \\
\text { = Femmes de la zone de comparaison }\end{array}$ & 6 & $\begin{array}{l}\text { Les hommes non exposés à l'intervention, vivant dans } \\
\text { les villages de comparaison = Hommes de la zone de } \\
\text { comparaison }\end{array}$ \\
\hline
\end{tabular}

\footnotetext{
${ }^{1}$ Cette dénomination ne correspond pas exactement à la réalité puisque certaines zones font partie de la province du Zoundwéogo, la province voisine.

${ }^{2}$ Un nouveau centre de santé et de promotion sociale a été construit dans le village de Sitenga. Mais l'aire de santé n'était pas définie au moment de l'évaluation finale.
} 
Les effets de l'intervention sur les indicateurs ont été mesurés en interrogeant et en comparant ces six groupes différents.

\section{Collecte des données et Analyse}

Deux approches ont été utilisées dans la collecte des informations de l'étude de base de 2001 . Il s'agit de l'étude qualitative et de l'étude quantitative. La première a consisté à mener des entretiens individuels approfondis et des groupes de discussion dirigée (focus groups) dans 8 villages des aires sanitaires de Béré, Bindé et Sondré. Le public cible était composé des potentiels participants inscrits sur les listes, les conjoints des femmes inscrites et des personnes de la population générale (filles, hommes, femmes), mais aussi des personnes ressources telles les responsables administratifs villageois, les chefs de village, les représentants des hommes et des femmes, les agents de santé. Les critères d'âge et de situation matrimoniale ont été retenus pour diversifier les informations. Les facilitateurs ${ }^{1}$ ont été interrogés à la fin du programme éducatif.

La collecte des données quantitatives de base s'est effectuée à la fois auprès des potentiels participants et des participants réels. On y ajoutera les non-participants de la zone expérimentale et les non-participants de la zone de comparaison.

Pour tester l'impact du programme d'éducation et le processus de mobilisation sociale sur les volontés des membres de la communauté d'abandonner la pratique de l'excision, les filles âgées de 0 à 10 ans au moment de l'enquête sont systématiquement incluses dans l'étude. Des questions sont posées aux mères sur le statut d'excision de chaque fille à chaque enquête. Les proportions de filles non excisées sont comparées aussi bien dans la zone expérimentale que dans la zone de comparaison.

Dans la zone expérimentale, les participants ont été interviewés chez eux à l'aide des listes établies pour la formation. Quant aux non-participants, 10 couples par village ont été interrogés. Ces derniers ont été sélectionnés dans les concessions qui ne comportaient aucun participant et dans chaque concession, un seul couple non-participant ${ }^{2}$ a été retenu. Pour mener à bien cette tâche, l'aide des responsables administratifs villageois (RAV ou délégués) a été précieuse.

Afin de mesurer les effets du programme d'éducation et le processus de mobilisation sociale contre l'excision sur la vie du couple, les conjoints des femmes participantes (eux-mêmes non participants) ont été inclus dans l'étude. Leur identification s'est effectuée sur le terrain, non seulement avec l'aide des responsables administratifs villageois et des responsables du comité villageois de gestion mis en place par le programme, mais également avec le concours des femmes participantes elles-mêmes. Ces conjoints n'ayant pas participé au programme ont ainsi été identifiés afin de leur administrer l'outil de collecte élaboré à leur intention.

Dans la zone de comparaison par contre, la sélection des dix couples s'est fait à l'étude de base de façon aléatoire ${ }^{3}$ à partir de la liste des chefs de ménages de chaque département. A

\footnotetext{
${ }^{1}$ Le terme de facilitateur communautaire a été utilisé pour désigner les formateurs des participants (hommes et femmes) du programme.

${ }^{2}$ Dans certains villages, il était impossible de réunir dix couples dans ces conditions, d'autant plus que la grande majorité des concessions ont au moins un(e) participant(e) dans le programme.

${ }^{3}$ A l'aide de la table des nombres aléatoires.
} 
l'étude finale, la sélection a été faite par pas de tirage selon le nombre de concessions dans chaque village ${ }^{1}$.

\section{Les outils de collecte}

Dans le cadre de l'étude qualitative, un guide d'entretien approfondi adressé aux leaders d'opinion et un guide pour les focus groups ont été utilisés. Un guide d'entretien a été élaboré également à l'intention des facilitateurs.

Quant à l'étude quantitative, trois types de questionnaires ont été utilisés : le questionnaire pour les femmes, celui des hommes et un questionnaire pour les conjoints des participantes. Les deux premiers s'adressent aussi bien aux participants qu'aux non-participants. Le dernier concerne uniquement les conjoints des participantes.

\section{La période de collecte}

La collecte des données de base a eu lieu en février 2001 et celle de l'étude post intervention en avril-mai 2002. Quant à l'étude finale, elle a démarré en fin avril dans la zone de comparaison avant de se poursuivre en zone expérimentale en mai 2003, juste après la cérémonie de déclaration publique de Béré.

\section{Saisie et analyse des données}

Les données de l'étude qualitative ont été transcrites, traduites et saisies par la suite dans WORD avant d'être transférer sur le logiciel NUD*IST pour traitement et exploitation. La saisie des données quantitatives a eu lieu à l'aide du logiciel ISSA avant l'épuration et l'analyse qui ont été effectuées sur le logiciel SPSS/10.

L'analyse est comparative entre les périodes de collecte. Etant donné que lors de l'étude de base les personnes qui se sont déclarés participantes au programme n'ont pas dans la réalité toutes intégrées le programme, et que durant le déroulement du programme d'autres personnes ont intégré, les données de l'enquête de base sont présentées pour l'ensemble des femmes ou des hommes de la zone expérimentale. Les données de l'enquête intermédiaire et de l'enquête finale sont présentées séparément pour les participants et les non-participants. Une analyse séparée sur les conjoints ${ }^{2}$ des participantes a également été menée.

Le test de khi deux est utilisé pour mesurer l'association entre les variables. Pour beaucoup plus de clarté dans la présentation, les différences statistiquement significatives sont signalées par des astérix. Le seuil minimum de confiance retenu est 95\%. Pour la plupart des indicateurs, la comparaison est faite dans le sens suivant :

Tableau 2 : Comparaison des indicateurs

\begin{tabular}{|c|c|c|c|c|c|c|}
\hline \multicolumn{5}{|c|}{ Zone expérimentale } & \multicolumn{2}{|c|}{ Zone de comparaison } \\
\hline $\begin{array}{c}\text { Base } \\
\text { (An 2001) }\end{array}$ & $\begin{array}{r}\text { Inter } \\
\text { (An }\end{array}$ & diaire & $\begin{array}{r}\mathbf{F} \\
(\mathrm{AN}\end{array}$ & $\begin{array}{l}\text { al } \\
\text { D03) }\end{array}$ & $\begin{array}{c}\text { Base } \\
(\text { An 2001) } \\
\end{array}$ & $\begin{array}{c}\text { Final } \\
(\text { An 2003) }\end{array}$ \\
\hline Avant le programme & $A$ la fin $d t$ & ogramme & Un an après & programme & $\begin{array}{c}\text { Avant le } \\
\text { programme }\end{array}$ & $\begin{array}{l}\text { Un an après le } \\
\text { programme }\end{array}$ \\
\hline Femmes/Hommes & Participants & $\begin{array}{c}\text { Non } \\
\text { participants }\end{array}$ & Participants & $\begin{array}{c}\text { Non } \\
\text { participants }\end{array}$ & Femmes/Hommes & Femmes/Hommes \\
\hline $\mathbf{A}$ & B & C & D & $\mathbf{E}$ & $\mathbf{F}$ & G \\
\hline
\end{tabular}

\footnotetext{
${ }^{1}$ Dans chaque village, on s'informait sur le nombre de concessions qui composent celui-ci. Ce nombre est ensuite divisé par 10 et on obtient ainsi le pas de tirage pour le village en question.

${ }^{2}$ Beaucoup de différences existent entre les données des conjoints et celui des hommes.
} 
1. Comparaison entre enquête de base et enquête intermédiaire pour les femmes et les hommes ayant bénéficié du programme (A vs B);

2. Comparaison à l'étude intermédiaire entre femmes et hommes ayant bénéficié du programme et femmes et hommes indirectement exposés parce que vivant dans le village (B vs C) ;

3. Comparaison des femmes et des hommes ayant bénéficié du programme à l'étude intermédiaire et à l'étude finale (B vs D) ;

4. Comparaison des femmes et des hommes indirectement exposés au programme à l'étude intermédiaire et à l'étude finale ( $\mathrm{C}$ vs $\mathrm{E})$

5. Comparaison entre enquête de base et les femmes et les hommes ayant bénéficié du programme à l'étude finale (A vs D) ;

6. Comparaison entre enquête de base et les femmes et les hommes indirectement exposés au programme à l'étude finale (A vs $\mathrm{E}$ );

7. Comparaison de la zone contrôle à l'enquête de base et à l'enquête finale (F vs G) ;

8. Tests statistiques de robustesse ( $\mathrm{z}$ test) entre l'enquête de base et l'enquête d'évaluation finale pour voir si l'intervention a eu un effet réel. Pour les indicateurs ayant connu une évolution positive dans les deux zones, ce test compare les différences d'écart entre les deux zones (AD/E vs F-G). Si cette marge des différences est supérieure à 1,96 (95\% de seuil de signification), l'intervention sera jugée comme ayant eu un effet.

Dans les différents tableaux, les résultats de l'étude finale ( $D$ et $E$ ) sont précédés ou suivis par des astérix. Ceux qui précèdent les résultats concernent les différences existantes entre l'étude intermédiaire et l'étude finale. Ceux qui suivent le résultat indiquent plutôt la différence existante entre l'étude de base et l'étude finale.

\section{Effectif des personnes enquêtées}

La répartition de la population interrogée dans le cadre de l'étude qualitative est présentée dans le tableau 3 suivant. Les effectifs sont présentés suivant le type de personne enquêté et selon la méthode utilisée.

\section{Tableau 3 : Répartition de la population interrogée à l'étude qualitative}

\begin{tabular}{llcc}
\hline \hline Qualité de l'enquêté & Populations cibles & $\begin{array}{c}\text { Entretiens } \\
\text { individuels }\end{array}$ & Focus groups \\
\hline \multirow{2}{*}{ Masculin } & Participants ${ }^{1}$ & 48 & 8 \\
& Conjoints de participantes & 24 & - \\
& Population générale & - & 8 \\
\hline \multirow{2}{*}{ Féminin } & Participantes & 48 & 8 \\
\hline \multirow{2}{*}{ Personnes ressources } & Conjointes de participants & 24 & - \\
& Population générale (femmes, filles) & - & - \\
& Infirmiers et autres agents de santé & 16 & - \\
\hline Facilitateurs & Représentant des femmes, des hommes, des jeunes & 8 & - \\
\hline Total & & 24 & $\mathbf{2 4}$ \\
\hline \hline
\end{tabular}


Quant aux résultats de la collecte quantitative, ils sont présentés dans le tableau 4 suivant. Ces résultats sont comparés à ceux de l'enquête de base et à ceux des deux enquêtes d'évaluation. La répartition est faite par sexe et par statut de participation au programme. Au cours de l'enquête post intervention, seule la zone expérimentale était concernée. On note quelques disparités entre les deux dernières opérations de collecte, essentiellement dus aux mouvements de population (décès, émigration, mariage, déplacement dans les champs de cultures,...).

\section{Tableau 4 : La population d'étude selon les différentes périodes de collecte}

\begin{tabular}{lccc}
\hline Caractéristique des enquêtés & $\begin{array}{c}\text { BASE }^{\mathbf{1}} \\
\text { (Enquête de base) }\end{array}$ & $\begin{array}{c}\text { INTERMÉDIAIRE } \\
\text { (Enquête Post I) }\end{array}$ & $\begin{array}{c}\text { FINAL } \\
\text { (Enquête finale) }\end{array}$ \\
\hline Zone expérimentale & & & 578 \\
Femmes participantes & 497 & 626 & 167 \\
Femmes non-participantes & 165 & 163 & 448 \\
Hommes participants & 515 & 465 & 169 \\
Hommes non-participants & 182 & 193 & 281 \\
Conjoints des femmes participantes & 315 & 295 & 1296 \\
Filles en vie & 942 & 1261 & 228 \\
\hline Zone de comparaison & & & 229 \\
Femmes & 226 & - & 464 \\
Hommes & 227 & - & \\
Filles en vie & 455 & & \\
\hline \hline
\end{tabular}

\section{Taux de couverture de l'enquête finale}

Les principales cibles de l'étude que sont les participants ont dans leur grande majorité été interrogées à chaque opération de collecte des informations (Cf. tableau 5 ci-dessous). En effet, $69 \%$ des femmes participantes et $75 \%$ des hommes participants ont été interrogés pour la troisième fois consécutive. Chez les conjoints, il s'agit de 56\% d'entre eux. Quant aux autres personnes non-participantes, la proportion de celles qui ont été visitées trois fois est faible, puisque les deux échantillons étaient constitués d'une manière indépendante. L'objectif de renforcement des capacités de tout le village par la circulation de l'information étant testé dans ces conditions. Les autres perdus de vue chez les participants sont expliqués par l'absence (voyage pour la plupart) de certains enquêtés au moment de l'enquête. Avec le programme d'éducation qui s'est déroulé en deux phases ${ }^{3}$, certains cas de sortie mais également d'entrée dans le programme ont été signalés après la première phase. Chez les femmes on a aussi enregistré des cas de déplacement pour raison de mariage.

\footnotetext{
${ }^{1}$ La distinction entre participants et non participants y est fictive. Aucune distinction ne sera faite entre eux puisqu'il s'agit de l'éventuelle participation au programme des hommes et des femmes s'étant inscrits pour la formation avant même le début des cours.

${ }^{2}$ Il s'agit des conjoints des femmes qui elles participent au programme. Par contre, ces conjoints ne participent pas à la formation

${ }^{3} \mathrm{La}$ formation a été dispensée en deux temps pour tenir compte de la période hivernale
} 
Tableau 5 : Régularité (en \%) de la participation des enquêtés lors des différentes collectes d'information

\begin{tabular}{lcccc}
\hline \hline Caractéristique des enquêtés & Une fois & Deux fois & Trois fois & Effectifs \\
\hline Zone expérimentale & & & & \\
Femmes participantes & 4 & 27 & 69 & $\mathbf{5 7 8}$ \\
Femmes non participantes & 67 & 23 & 9 & $\mathbf{2 2 8}$ \\
Hommes participants & 2 & 24 & 75 & $\mathbf{4 4 8}$ \\
Hommes non participants & 59 & 23 & 18 & $\mathbf{1 6 9}$ \\
Conjoints des femmes participantes & 15 & 30 & 56 & $\mathbf{2 8 1}$ \\
\hline Zone de comparaison & & & & $\mathbf{2 2 8}$ \\
Femmes non participantes & 78 & 22 & na & $\mathbf{2 2 9}$ \\
Hommes non participants & 73 & 28 & na
\end{tabular}

\section{Limites de l'étude}

Le thème de l'étude: Malgré la confiance instaurée par la longue présence sur le terrain et la facilité des habitants des deux zones à parler de l'excision, la délicatesse du thème peut toujours entraîner une certaine réserve chez certains enquêtés. De plus, la loi interdisant la pratique de l'excision crée toujours une certaine méfiance et peut provoquer une rétention de l'information ou une grande disposition à donner des réponses qui restent conformes au contexte de celle-ci, notamment en ce qui concerne l'approbation de la pratique et l'intention d'exciser les filles.

L'état d'excision des filles de 0-10 ans : Les informations recueillies à ce niveau relèvent des déclarations des mères. Aucun examen physique n'a été mené sur les filles afin de vérifier les déclarations. La prévalence de l'excision ainsi obtenue au sein de ces filles est uniquement basée sur les déclarations des mères.

Les fluctuations de la population enquêtée : Dans bien des cas, certaines personnes (hommes et femmes) se sont déplacées, après avoir participé à l'une des collectes de données, tandis que d'autres qui étaient absents au cours de l'une des collectes y sont présents pour une autre collecte. De ce fait, certaines personnes n'ont pas fait partie de l'enquête à mi-parcours et dans l'évaluation finale. Pourtant, ceux qui sont interrogés plus tard pourraient influencer les résultats. Cependant, au regard de la faiblesse du nombre de déperdition et de complément d'effectif, on est en droit d'affirmer que cela aura une influence limitée sur les résultas.

L'évolution des questions : Au cours des différentes collectes d'information, il y a eu une évolution des questions posées aux enquêtés (retrait, rajout, modification de questions). Ceci est lié au souci de recueillir des informations pertinentes et fiables. Dans ces conditions, certaines comparaisons se sont avérées difficiles. Cette situation a limité parfois le nombre d'indicateurs pouvant être comparé.

\section{Caractéristiques de la population d'étude}

Les femmes dans leur ensemble diffèrent quelque peu suivant le critère de l'âge entre l'étude de base et les autres études d'évaluation, sauf dans la zone de comparaison où il n'y a pas de différence significative (Cf. tableaux en annexe). Avant le programme, il y avait un peu plus de jeunes femmes inscrites mais celles ayant réellement participé sont sensiblement plus âgées. L'âge médian était de 29 ans à l'étude de base contre 31 ans à l'enquête post 
intervention. Celles de la zone de comparaison sont plus âgées puisque l'âge médian y est de 33 ans à l'étude de base et de 35 ans à l'étude finale. Cette évolution est normale car l'échantillon a vieilli avec les années (2001-2003). Dans l'ensemble des deux zones, les femmes les plus représentées sont celles qui ont un âge compris entre 20 et 40 ans.

Chez les hommes par contre, on observe une différence significative entre les participants et les non participants $(\mathrm{p}<.01)$, ceux du programme étant plus jeunes que les autres. Entre l'étude de base et l'étude finale, l'âge médian des hommes est pratiquement le même. En zone de comparaison, on a enregistré des hommes significativement plus âgés avec une différence d'âge de 5 ans entre les deux échantillons ( $<<0,01)$. Chez les conjoints des participantes, leur répartition par âge reste significativement la même sur les trois périodes. L'âge médian oscille entre 47 et 48 ans.

Dans les deux zones, les femmes comme les hommes (y compris les conjoints) sont presque tous sans instruction. Néanmoins, les hommes demeurent relativement plus instruits que les femmes. En effet, que ce soit les enquêtés de l'étude de base comme ceux de l'enquête intermédiaire et ceux de l'étude finale, au moins 9 femmes sur 10 sont sans niveau d'instruction. Chez les hommes, les non instruits dépassent également $80 \%$. Pour ceux et celles qui ont fréquenté l'école, le primaire est quasiment le niveau le plus élevé atteint. On notera également que c'est parmi les participants au programme (femmes et hommes) que l'on rencontre le plus de personnes ayant fréquenté l'école. Chez les hommes par exemple, on observe un niveau d'instruction plus élevé chez les participants $(\mathrm{p}<.01)$. Les conjoints sans instruction représentent près de $89 \%$.

Aucune évolution n'est observée en ce qui concerne la religion. Dans pratiquement les mêmes proportions depuis l'étude de base, la proportion des musulmans est plus importante dans la zone expérimentale, suivie par les catholiques, les protestants et les autres, aussi bien chez les hommes que chez les femmes. Dans la zone de comparaison par contre, la proportion des catholiques apparaît plus importante. Aucune différence significative n'est observée au fil du temps.

Les femmes de la zone expérimentale sont dans des foyers polygames tandis que celles de la zone de comparaison sont pour la plupart dans un foyer monogame, suivant en ceci la religion dominante de chacune des zones. En effet la polygamie est plus fréquente dans la religion musulmane que dans la religion chrétienne. Chez les femmes, on enregistre au fil du temps une différence significative selon la situation matrimoniale, avec un peu plus de femmes polygames parmi les participantes au programme. Chez les hommes par contre, on a enregistré presque autant de polygames que de monogames ; $2 \%$ des hommes participants sont célibataires et $11 \%$ sont soit veufs ou séparés. Dans la zone de comparaison, seuls des couples ont été interrogés.

Les deux zones étant situées dans le plateau central mossi, on enregistre dans chaque cas une population homogène sur le plan ethnique $(100 \%)$.

En ce qui concerne l'alphabétisation et la formation antérieure, on enregistre une différence significative pour les deux variables entre les participants (hommes comme femmes) et les non participants $(\mathrm{p}<.01)$. On note ainsi dans les deux échantillons des femmes et des hommes de la zone expérimentale, la même répartition que celle de l'étude intermédiaire où les participants sont plus instruits que les autres, avec plus de $30 \%$ de femmes participantes alphabétisées ( $48 \%$ chez les hommes) contre $10 \%$ chez les autres femmes ( $15 \%$ chez les 
hommes) du village. En zone de comparaison, les alphabétisés représentent un peu plus de $20 \%$ des femmes et $31 \%$ des hommes. Ils sont $87 \%$ des femmes et $89 \%$ des hommes participants à avoir bénéficié de formation antérieure. Déjà à l'étude intermédiaire, on a assisté à une surestimation au niveau de ces deux indicateurs compte tenu de l'importance que ces femmes et ces hommes accordent à la formation dispensée dans le cadre de ce programme de renforcement et qui est déjà considérée comme une formation antérieure. Les conjoints des femmes participantes quant à eux sont très peu alphabétisés $(20 \%$ dans l'ensemble) et n'ont pas beaucoup bénéficié de formation antérieure.

\section{Appréciation du programme par les communautés}

Il faut noter que les classes pour les hommes ont démarré après celles des femmes, pour permettre aux facilitateurs de mieux s'organiser. Les hommes n'ont donc pas pu bénéficier de tous les modules. Avant la mise en œuvre du programme, les populations avaient un certain nombre d'attentes. Lors de l'enquête de base et de l'enquête post intervention, une série de questions a permis de saisir les attentes, la participation et l'intérêt pour le programme. Cette analyse ne porte que sur les participants au programme de la zone d'intervention.

\section{Participation et intérêt pour la formation}

\section{Le niveau de participation des populations}

Il était prévu que 30 femmes et 30 hommes seraient sélectionnés dans chaque village. A la fin du programme, on a enregistré une fluctuation des effectifs dans les villages. Tous ceux qui étaient inscrits au départ n'y ont pas réellement participé. Par contre, de nouveaux participants ont intégré le groupe dans certains villages. Cette fluctuation des effectifs est observable aussi bien chez les hommes que chez les femmes.

Un engouement manifeste est remarquable chez les femmes des 23 villages, où un peu plus de la moitié des villages ont eu plus de participantes que prévues. En exemple, le nombre des participantes réelles dans le chef lieu de département de Bindé a même atteint le double du nombre d'inscrit au départ. Chez les hommes par contre, le nombre de participants réels est inférieur au nombre prévu, dans presque tous les villages. A Bindé par exemple, seuls 18 personnes étaient inscrites sur la liste établie à la fin du programme.

\section{Le suivi des modules du programme}

Les données indiquent que $63 \%$ des femmes et $60 \%$ des hommes déclarent avoir suivi tous les modules. La principale raison évoquée pour la non participation des hommes est le manque de temps, les voyages, les maladies et les décès dans la famille. Les femmes évoquent en premier lieu les problèmes de maladie, suivi des voyages, le manque de temps et les décès dans la famille. Des femmes ont aussi mentionné leur grossesse et leur accouchement. Pour $8 \%$ d'entre elles, certains modules sont destinés aux hommes. Chez les hommes, le suivi des modules du programme est statistiquement lié à l'âge $(p<.01)$ et non au niveau d'instruction et au statut matrimonial, ni à la formation antérieure (alphabétisation ou autre). Ainsi, les hommes plus âgés ont un niveau de suivi plus élevé que ceux qui sont moins âgés. Les activités économiques des plus jeunes (commerce notamment) et leur plus grande liberté de mouvement en font des personnes très mobiles, au gré des différents marchés de la zone, ce qui perturbe la régularité aux séances. Chez les femmes par contre, il n'y a aucune relation entre le suivi de l'ensemble des modules et les caractéristiques sociodémographiques. Selon les facilitateurs, la régularité et l'assiduité au cours est allée en 
décroissant du début vers la fin du programme. Les marchés et les événements sociaux sont les principales justifications de l'absentéisme des participants ${ }^{1}$.

Le niveau d'intérêt porté sur le programme

L'intérêt vis-à-vis des modules du programme est variable (graphique 1). Pour tous les participants, l'hygiène et la prévention de la maladie ainsi que la santé de la femme ont été les thèmes les plus appréciés. Cependant l'ordre des intérêts diffère selon le sexe, les femmes $(82 \%)$ étant beaucoup plus intéressées par le module sur la santé de la femme. Les hommes quant à eux (77\%) préfèrent l'hygiène et la prévention de la maladie. Les deux autres modules (les droits humains et le processus de résolution des problèmes) ont moins intéressé les participants. Moins de $1 \%$ des participants affirment qu'aucun des modules ne les a intéressés.

\section{Graphique 1 : Proportion des participants suivant les modules qu'ils jugent intéressants}

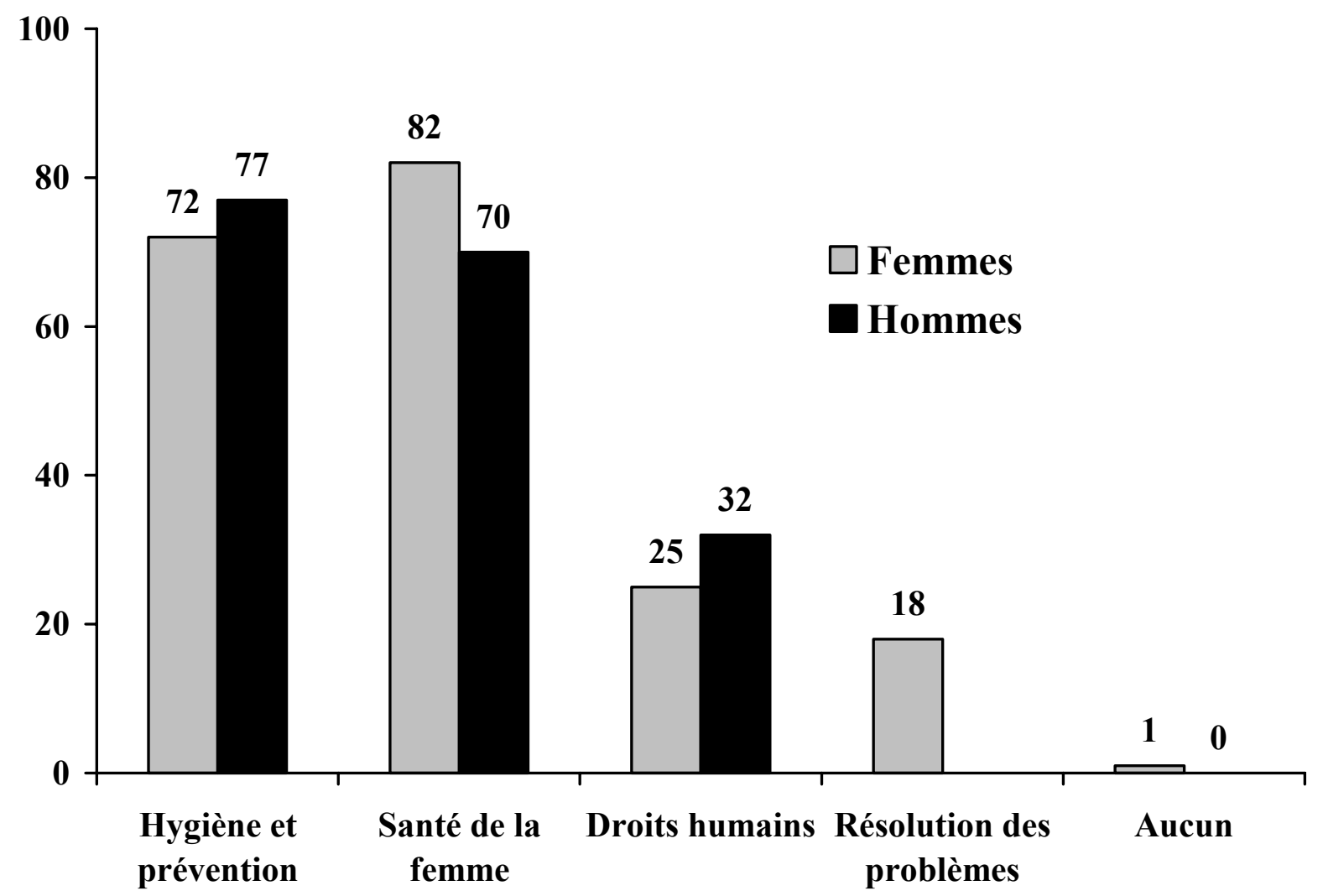

Les informations sur la santé, l'hygiène et la prévention de la maladie touchent plus les populations dans leur vie quotidienne. A l'étude de base, hommes et femmes de la zone ont particulièrement souligné que les problèmes de droit relevaient exclusivement de la responsabilité de l'Etat et du gouvernement. Les questions de droits étaient donc très loin de leurs préoccupations ${ }^{2}$. Les femmes avaient estimé qu'elles auraient un rôle personnel à jouer lorsqu'il s'agirait de résoudre des problèmes d'environnement sain, d'alimentation et

\footnotetext{
${ }^{1}$ Tamini et al. (2002) : Rapport qualitatif auprès des facilitateurs communautaires, rapport non publié

${ }^{2}$ Congo et al. (2001) : Rapport de l'étude de base, pages 30-31
} 
d'éducation. Les hommes avaient mentionné les problèmes d'eau potable, de santé, d'alimentation, de travail et d'accès au crédit.

Les attentes des populations

Dans l'ensemble, les hommes comme les femmes avaient une réelle envie d'améliorer leur niveau de connaissance (tableau 6). Neuf personnes sur 10 estiment que leurs attentes ont été comblées, la principale étant l'amélioration des connaissances. Certaines attentes sont plutôt liées à l'amélioration de pratiques de vie : $6 \%$ des femmes et $3 \%$ des hommes avaient espéré trouver un emploi grâce à cette

\section{Tableau 6 : Répartition des participants suivant leurs attentes avant la formation}

\begin{tabular}{lcc}
\hline \hline \multicolumn{1}{c}{ Attentes } & $\begin{array}{c}\text { Femmes } \\
(\mathbf{( \% )}\end{array}$ & $\begin{array}{c}\text { Hommes } \\
(\mathbf{\%})\end{array}$ \\
Améliorer les connaissances & 94 & 94 \\
Connaître les règles d'hygiène & 22 & 16 \\
Connaître les règles de vie en société & 14 & 20 \\
Trouver un emploi & 6 & 3 \\
Connaître les règles sanitaires & 5 & 11 \\
Trouver /recevoir de l'argent & 5 & 5 \\
Apprendre mes droits & 4 & 6 \\
Autres & 4 & 4 \\
Aucune & 2 & 2 \\
\hline \hline
\end{tabular}
formation. D'autres attentes ont été enregistrées tels que l'obtention de forages, de l'aide alimentaire et l'augmentation du nombre d'agents de santé ${ }^{1}$.

Les apports du programme

\section{Graphique 2 : Les apports du programme selon les participants}

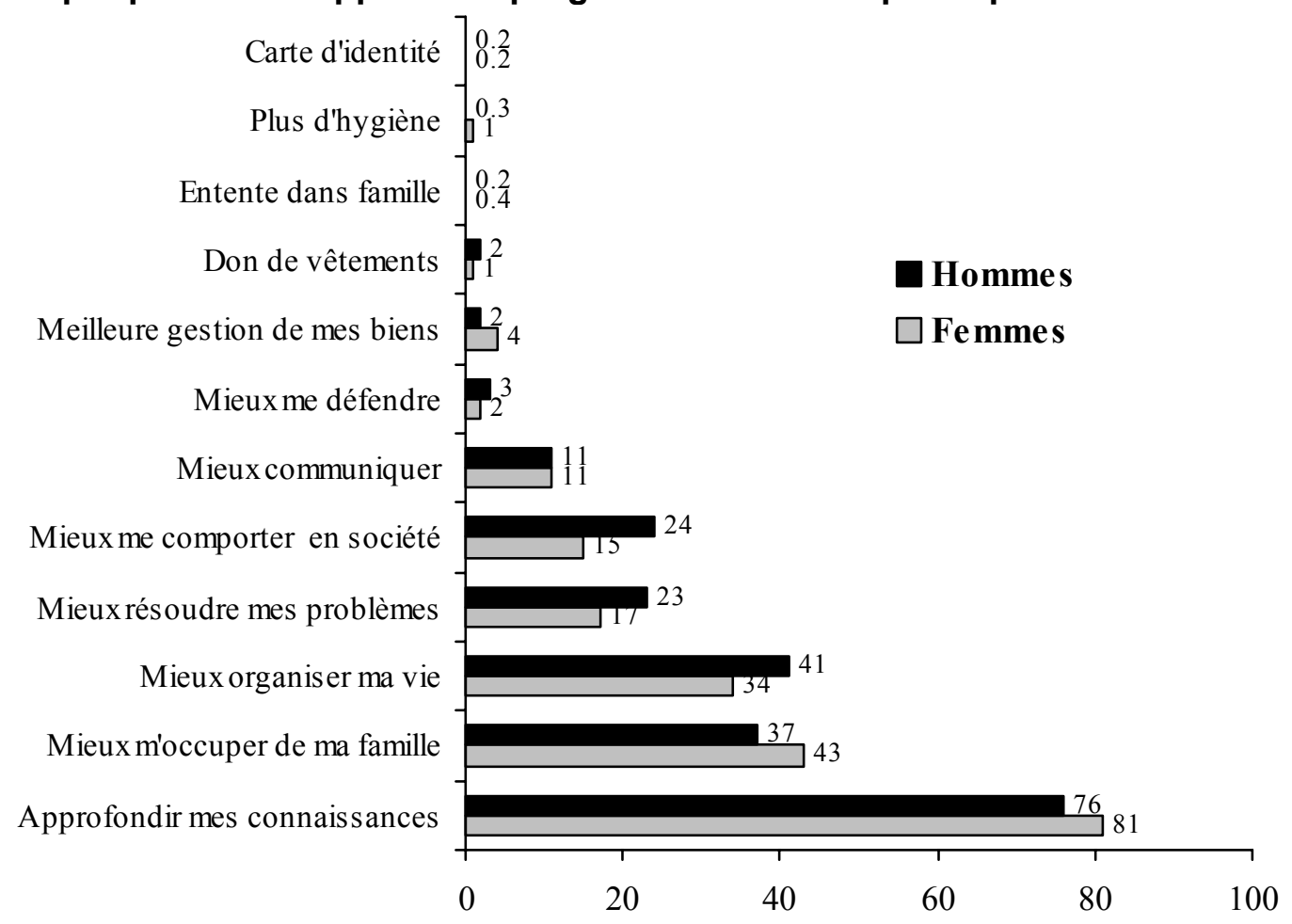

\footnotetext{
${ }^{1}$ Ces attentes sont classifiées dans la catégorie "autres" compte tenu de la faiblesse de l'effectif des hommes et de mention.
} 
Pour les participants, le programme a été bénéfique (graphique 2). Le premier apport mentionné est celui des connaissances. Ils soulignent également le fait d'être outillé pour mieux s'occuper de la famille, pour mieux organiser leur vie, pour résoudre leurs problèmes, pour mieux se comporter et communiquer davantage. La communication s'articule surtout autour de la possibilité et la volonté de parler ouvertement de l'excision, d'en débattre et de faire partager ces nouvelles connaissances à d'autres personnes.

\section{Les insuffisances constatées par les participants}

Dans leur ensemble, près de $80 \%$ des participants estiment qu'il n'y a aucune insuffisance à mettre au compte du programme. Bien que les insatisfaits soient proportionnellement faibles, près de $20 \%$ des participants signalent dans l'ordre, le manque de mesure

d'accompagnement, les difficultés dans le suivi des cours, le manque de classes, le manque de cours d'alphabétisation, la mauvaise organisation, le changement de facilitateur, le non isolement des classes et les problèmes d'assimilation des leçons.

\section{Le partage de l'expérience acquise}

Dans la mise en œuvre du programme, un système de parrainage a été développé pour transmettre aux autres les informations reçues. Chaque participant(e) doit choisir un ami dans le village (cette personne n'étant pas participant(e)) avec qui il/elle partage toutes les informations reçues dans les classes. Dans l'ensemble l'information semble avoir été partagée avec des ami(e)s, le conjoint et la famille (graphique 3).

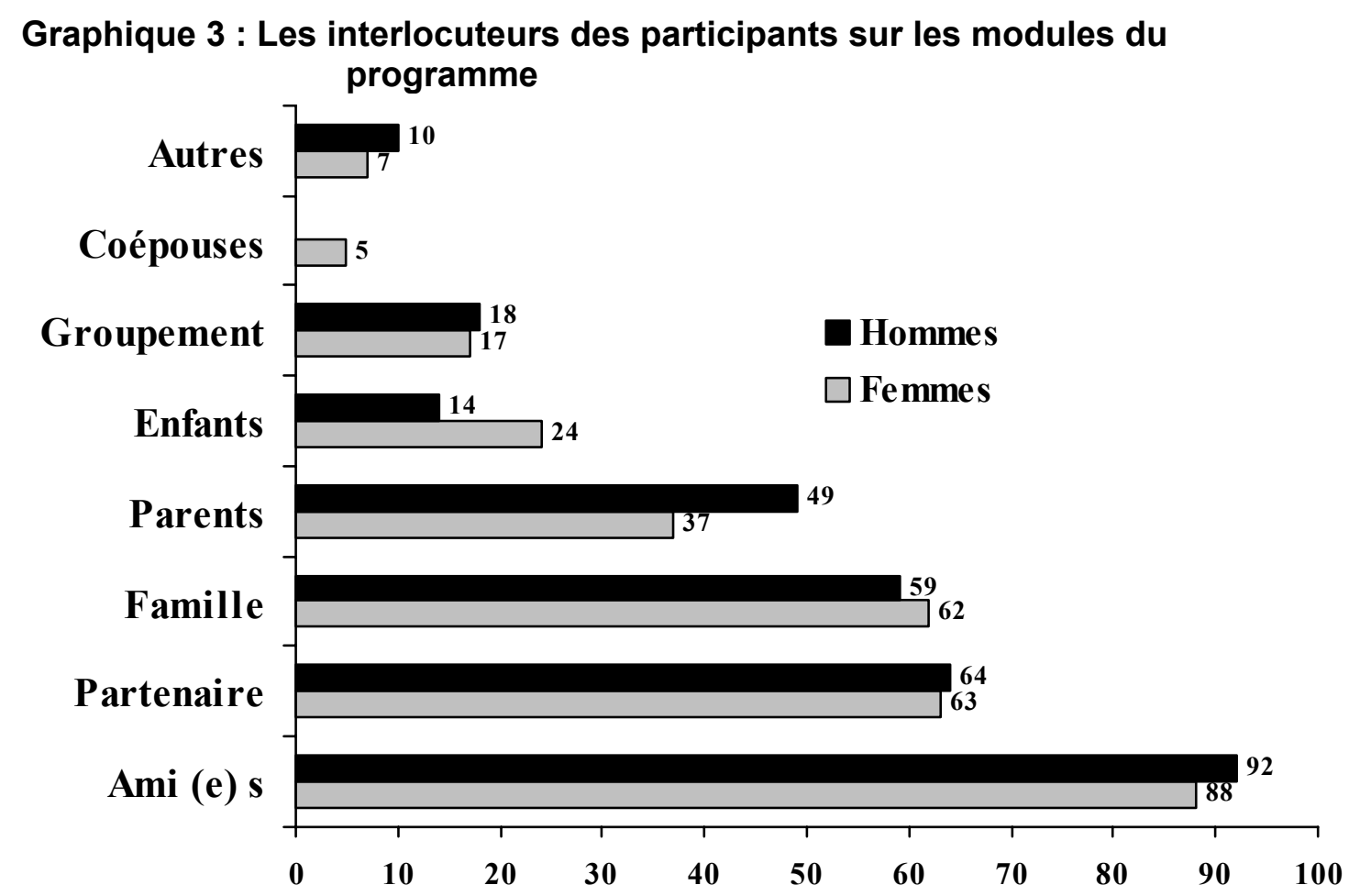

Les thèmes d'information et de discussion en dehors des classes

De façon générale, les sujets liés à la santé sont les plus discutés (tableau 7). On notera en effet dans l'ordre, les règles d'hygiène, les IST/VIH/SIDA, suivis des modes de prévention des maladies et de la contraception. 


\begin{tabular}{lcccc}
\hline \hline & \multicolumn{2}{c}{ Femmes } & \multicolumn{2}{c}{ Hommes } \\
\cline { 2 - 5 } & Participantes & $\begin{array}{c}\text { Non } \\
\text { participantes }\end{array}$ & Participants & $\begin{array}{c}\text { Non } \\
\text { participants }\end{array}$ \\
\hline \hline Les sujets de discussion & & & & \\
\hline \hline IST/VIH/SIDA & 90 & 69 & 97 & 91 \\
Mode de prévention des maladies & 85 & 63 & 89 & 84 \\
Contraception & 84 & 47 & 82 & 69 \\
Règles d'hygiène & 90 & 67 & 92 & 80 \\
Droits humains & 69 & 26 & 76 & 48 \\
Rôles dans la famille & 69 & 50 & 75 & 62 \\
\hline Effectifs & $\mathbf{6 2 6}$ & $\mathbf{1 6 3}$ & $\mathbf{4 6 5}$ & $\mathbf{1 9 3}$ \\
\hline \hline
\end{tabular}

Les participants sont ceux qui discutent le plus de ces sujets et ces nouvelles connaissances renforcent leur capacité à communiquer. Cette facilité peut être une source d'admiration et de faire valoir, de sorte que ces participants peuvent devenir, des modèles pour les autres.

\section{Contribution du VEP sur les Connaissances, Attitudes et Comportements}

\section{Les Droits Humains}

\section{Connaissance spontanée des droits humains}

Le graphique 4 montre que deux fois plus d'hommes et de femmes participants au programme disent avoir déjà entendu parler des droits humains lors de l'étude intermédiaire. Chez les hommes non-participants le niveau de connaissance reste le même mais chez les femmes non-participantes il y a une baisse significative due au biais de sélection. Lors de l'évaluation finale, ces proportions augmentent encore significativement chez les hommes et les femmes participants et non participants, alors qu'aucun changement n'est observé dans la zone de comparaison. 


\section{Graphique 4 : A entendu parler des droits humains ${ }^{1}$}

\section{Zone expérimentale}

Zone de comparaison

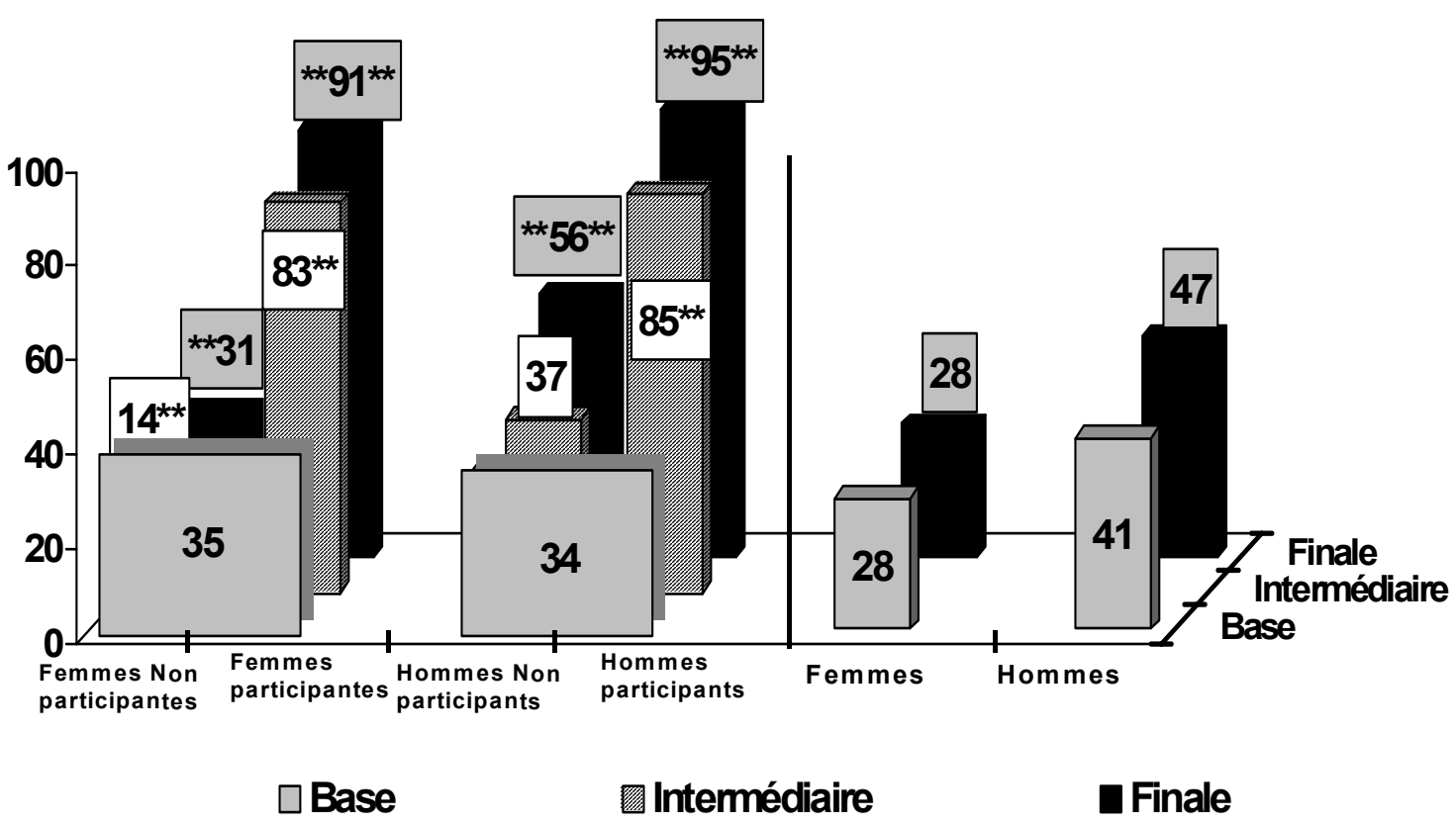

Chez les conjoints des femmes participantes (tableau 8), on observe une augmentation significative des indicateurs seulement à l'enquête finale. Cette évolution pourrait s'expliquer par l'augmentation des activités de mobilisation sociale (réunions villageoises, activités de renforcement des capacités acquises) menées dans la zone d'intervention à la fin du programme.

\section{Tableau 8 : Connaissances des conjoints des femmes participantes sur les droits} humains2

\begin{tabular}{|c|c|c|c|}
\hline \multirow[t]{2}{*}{ Indicateurs } & \multicolumn{3}{|c|}{ Zone expérimentale } \\
\hline & Base & Intermédiaire & Finale \\
\hline DROITS HUMAINS (N) & 315 & 195 & 281 \\
\hline A entendu parler des droits humains & 43 & 45 & $* * 72 * *$ \\
\hline Connaissant les droits après suggestion & 43 & 39 & $* * 60 * *$ \\
\hline Connaît des pratiques qui avantagent plus les hommes & 30 & 25 & 25 \\
\hline $\begin{array}{l}\text { Pas d'accord que certaines tâches soient uniquement } \\
\text { réservées aux hommes }\end{array}$ & 47 & 51 & $* 61 * *$ \\
\hline
\end{tabular}

Les différences significatives entre périodes dans chaque zone s'expriment ainsi : * $p<0,05 * * p<0,01$

\footnotetext{
${ }^{1}$ Les astérix placés avant l'indicateur de l'étude finale matérialisent la différence significative avec l'étude intermédiaire. Les astérix placés près l'indicateur concernent la différence avec l'étude de base.

${ }^{2}$ Rappelons que les astérix placés avant l'indicateur de l'étude finale matérialisent la différence significative avec l'étude intermédiaire. Les astérix placés après l'indicateur concernent la différence avec l'étude de base.
} 
Par conséquent, on peut dire que le programme a contribué à faire connaître les droits humains dans la zone expérimentale, surtout parmi les participants au programme d'éducation. Dans le reste de la communauté, ce sont surtout les hommes qui ont amélioré leur niveau de connaissance, la diffusion de l'information dans la communauté des femmes étant moindre. La relative baisse constatée chez les femmes non-participantes lors de l'enquête intermédiaire est due au biais de sélection après que l'échantillon a été scindé en deux, participants et non participants ; Les participants au programme (hommes et femmes) étant plus instruits que les autres. Il faut aussi remarquer que le système de parrainage recommandé par TOSTAN ne semble pas avoir fonctionné d'une manière optimale au Burkina. Les femmes non participantes ont augmenté leurs connaissances sur les droits une année après le programme, mais bien moins que les hommes. Il peut aussi y avoir l'effet de l'oubli à cause de la rupture due à l'hivernage. A la reprise des classes, les participants ont pu bénéficier d'un rappel des enseignements précédents, notamment ceux portant sur les droits humains.

\section{Reconnaissance de pratiques avantageant plus les hommes que les femmes}

Les femmes participantes se distinguent à l'étude intermédiaire puisque la proportion de celles qui reconnaissent les pratiques avantageant les hommes augmente significativement $(\mathrm{p}<.01)$. Chez les non-participantes et les hommes, on n'enregistre aucune différence avec l'étude de base (tableau 9). Les pratiques discriminatoires les plus citées sont : l'absence aux prises de décision, l'inégalité dans la prise de parole, celle de la liberté de mouvement, le partage de l'héritage et la non-scolarisation des filles. A l'étude finale, la proportion de personnes soutenant les connaître augmente sensiblement. Participants et non-participants ont cité en plus grand nombre ces pratiques discriminatoires. Cependant l'écart se maintient entre les participants et les non-participants, aussi bien chez les femmes que chez les hommes. Chez les conjoints par contre, aucune différence significative n'est observée au fil du temps.

\section{Tableau 9 : Niveau de connaissance et attitudes des hommes et des femmes sur les} droits humains

\begin{tabular}{|c|c|c|c|c|c|c|c|}
\hline \multirow{3}{*}{ Indicateurs } & \multirow[b]{3}{*}{ Base } & \multicolumn{4}{|c|}{ Zone expérimentale } & \multicolumn{2}{|c|}{$\begin{array}{c}\text { Zone de } \\
\text { comparaison }\end{array}$} \\
\hline & & \multicolumn{2}{|c|}{ Intermédiaire } & \multicolumn{2}{|c|}{ Final } & \multirow[b]{2}{*}{ Base } & \multirow[b]{2}{*}{ Final } \\
\hline & & Participants & $\begin{array}{c}\text { Non } \\
\text { participants }\end{array}$ & Participants & $\begin{array}{c}\text { Non } \\
\text { participants }\end{array}$ & & \\
\hline FEMMES & 662 & 626 & 163 & 578 & 167 & 226 & 228 \\
\hline $\begin{array}{l}\text { Connaît des pratiques qui avantagent plus } \\
\text { les hommes }\end{array}$ & 25 & $38^{* *}$ & 23 & $* * 48^{* *}$ & $* 34 *$ & 31 & $47 * *$ \\
\hline $\begin{array}{l}\text { On peut changer cette situation des } \\
\text { pratiques qui avantagent plus les hommes } \\
\text { que les femmes }\end{array}$ & 22 & $33^{* *}$ & $15^{*}$ & $* * 83 * *$ & $* * 70 * *$ & 26 & $60 * *$ \\
\hline $\begin{array}{l}\text { Ne pense pas que certains postes soient } \\
\text { uniquement réservés aux hommes }\end{array}$ & 32 & $66^{* *}$ & $43 *$ & $65^{* *}$ & $* 56 * *$ & 40 & $54 * *$ \\
\hline HOMMES & 697 & 465 & 193 & 448 & 169 & 227 & 229 \\
\hline $\begin{array}{l}\text { Connaît des pratiques qui avantagent plus } \\
\text { les hommes }\end{array}$ & 27 & 31 & 22 & $* * 44 * *$ & $* * 35 *$ & 26 & 43 \\
\hline $\begin{array}{l}\text { On peut changer cette situation des } \\
\text { pratiques qui avantagent plus les hommes } \\
\text { que les femmes }\end{array}$ & 22 & 27 & 16 & $* * 41 * *$ & $* * 28$ & 21 & $31 *$ \\
\hline $\begin{array}{l}\text { Ne pense pas que certaines tâches soient } \\
\text { uniquement réservées aux hommes }\end{array}$ & 45 & $51 *$ & $54 *$ & $* * 66 * *$ & $59 * *$ & 52 & 56 \\
\hline
\end{tabular}

\footnotetext{
${ }^{1}$ On a pris comme dénominateur toutes les femmes
} 
En zone de comparaison, seule la proportion des femmes qui affirment connaître les pratiques discriminatoires a connu une évolution significative entre l'étude de base et l'étude finale. Les résultats du test de comparaison effectué sur les deux zones $(\mathrm{z}=0,79)$ ne permet pas d'attribuer l'évolution observée au programme d'éducation.

A l'étude intermédiaire 33\% des femmes participantes affirmaient que la discrimination envers les femmes pouvait changer, compare à $22 \%$ à l'étude de base. Aucun changement notable n'est observé chez les non-participantes et les hommes. A l'étude finale on observe un changement significatif chez les femmes. La proportion de femmes participantes et non participantes désormais convaincues de la possibilité de changer la société dépasse de près de 4 fois celle de l'étude de base. Chez les hommes, une légère différence est aussi observée, mais uniquement parmi les participants.

En zone de comparaison, il y a également une différence significative aussi bien chez les femmes que chez les hommes, mais les proportions sont plus élevées chez les femmes. Cependant le test de comparaison entre les deux zones $(\mathrm{z}=2,78)$ montre que la zone expérimentale a une évolution significativement différente de la zone de comparaison.

En conclusion, on retient que le niveau de connaissance en matière de droits humains a beaucoup évolué, aussi bien chez les hommes, y compris les conjoints, que chez les femmes, grâce notamment à l'action du programme d'éducation, puisque la zone de comparaison ne change pas. Par contre les pratiques discriminatoires sont mieux connues dans les deux zones. Le programme d'éducation, et les activités de mobilisation sociale ont donné l'occasion aux femmes de s'exprimer, non seulement dans les cercles restreints tels que les réunions mais également en publique, devant plusieurs chefs religieux et coutumiers de la zone. Cela peut avoir eu pour effet de convaincre davantage les femmes que beaucoup de choses peuvent changer, notamment en ce qui concerne les discriminations. Chez les hommes, il y a également une évolution significative. L'opinion sur les perspectives de changement des discriminations évolue positivement chez les femmes et les hommes participants au programme et aussi chez les femmes non participantes. Cependant la zone de comparaison évolue aussi, ce qui montre que des changements endogènes ont lieu dans toute la province. 


\section{Les violences faites aux femmes}

Certains types de violences sont souvent exercés contre les femmes. Il s'agit notamment des violences sexuelles, physiques, morales et/ou psychologiques, verbales et économiques.

\section{Graphique 5 : Connaissance d'au moins une des formes de violences}

\section{Zone expérimentale}

\section{Zone de comparaison}

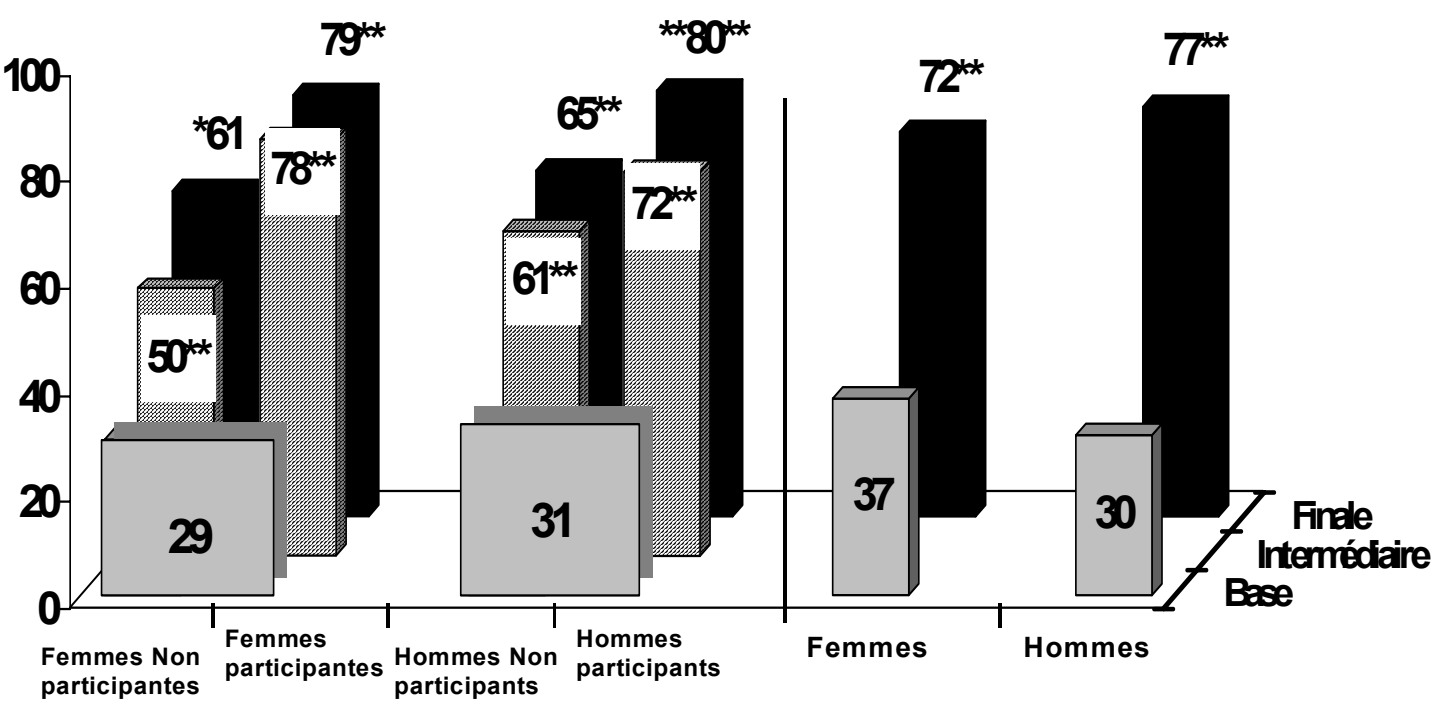

Base

u Intermédiaire

口Finale

La connaissance des formes de violences a progressé significativement dans les deux zones et parmi tous les groupes. Si seulement un tiers des femmes et des hommes de la zone expérimentale reconnaissaient à l'étude de base au moins une des formes de violences citées ci-dessus, ils représentent à l'étude finale près de trois quarts des participants et un peu plus de $60 \%$ des non-participants (graphique 5). En zone de comparaison, on assiste également à une évolution dans la reconnaissance des formes de violence, aussi bien parmi les hommes que les femmes. Ainsi des changements endogènes touchent toute la région, même si le test de comparaison des évolutions chez les femmes montre que la zone d'intervention a évolué plus fortement $(z=2,12)$. Par contre le niveau d'évolution des hommes est comparable entre les deux zones.

Une proportion plus importante de femmes et d'hommes reconnaît l'existence de violences dans la communauté après le programme (tableau 10). Le programme semble avoir permis de libérer la parole et d'avoir une meilleure reconnaissance des actes de violence. Une année après, à l'évaluation finale les niveaux déclarés baissent significativement (43\%-44\%). La zone de comparaison ne montre pas de changements au cours du temps.

Vécu et approbation des violences

Dès la fin du programme, $11 \%$ des femmes participantes et $9 \%$ des non-participantes de la zone expérimentale ont soutenu avoir vécu au moins une de ces formes de violences (tableau 
10) contre $7 \%$ à l'étude finale, soit une baisse significative de $34 \%$ chez les femmes participantes $(\mathrm{p}<.05)$. Dans le même temps, les femmes qui subissent les différentes formes de violence dans la zone de comparaison représentent $16 \%$. La différence est significative entre les deux zones à l'enquête finale.

Tableau 10 : Connaissance et attitude des hommes et des femmes face aux violences faites aux femmes

\begin{tabular}{|c|c|c|c|c|c|c|c|}
\hline \multirow{3}{*}{ Indicateurs } & \multirow[b]{3}{*}{ Base } & \multicolumn{3}{|c|}{ Zone expérimentale } & & \multirow{2}{*}{\multicolumn{2}{|c|}{ Zone de comparaison }} \\
\hline & & \multicolumn{2}{|c|}{ Intermédiaire } & Final & & & \\
\hline & & Participants & $\begin{array}{c}\text { Non } \\
\text { participants }\end{array}$ & Participants & $\begin{array}{c}\text { Non } \\
\text { participants }\end{array}$ & Base & Final \\
\hline FEMMES & 662 & 626 & 163 & 578 & 167 & 226 & 228 \\
\hline Cite au moins une forme de violence & 29 & $78 * *$ & $50 * *$ & $79 * *$ & $* 61 * *$ & 37 & $72^{* *}$ \\
\hline Existe dans la communauté1 & 51 & $89^{* *}$ & $82 * *$ & $* * 43 * *$ & $* * 44$ & 65 & 66 \\
\hline Vécu personnel & - & 11 & 9 & *7 & 7 & - & 16 \\
\hline Désapprobation des violences2 & 99 & 100 & 99 & $* * 97 * *$ & 98 & 100 & $96 * *$ \\
\hline HOMMES & 697 & 465 & 193 & 448 & 169 & 227 & 229 \\
\hline Cite au moins une forme de violence & 31 & $72^{* *}$ & $61 * *$ & $* * 80 * *$ & $65^{* *}$ & 30 & $77 * *$ \\
\hline Existe dans la communauté & 60 & $89 * *$ & $86 * *$ & $* * 49 * *$ & $* * 44 * *$ & 64 & 67 \\
\hline Désapprobation des violences & 99 & 99 & 99 & $* * 95 * *$ & $* 95 * *$ & 99 & $89 * *$ \\
\hline
\end{tabular}

La désapprobation des violences est pratiquement universelle.

Les changements intervenus à propos des violences

Les disputes, les bagarres et le fait que la femme soit mieux écoutée sont identifiés par les populations comme étant les changements intervenus depuis la mise en œuvre du programme (graphique 6). Cette opinion est partagée par l'ensemble des femmes et des hommes.

En conclusion on retient à propos des violences que le niveau de reconnaissance des formes de violence contre les femmes a évolué dans toute la province, montrant des changements endogènes importants. Cependant chez les femmes de la zone expérimentale l'évolution est plus importante et peut être mise sur le compte du programme. Par contre les hommes montrent le même niveau de changement dans les deux zones. L'augmentation du niveau de connaissance en zone de comparaison peut également être expliquée par les actions multiformes des ONG et associations qui par leurs activités luttent contre ces formes de violence. Les attitudes et les comportements violents ont montré une forte progression dans la zone d'intervention avant de baisser significativement. On peut conclure que le programme a permis une meilleure dénonciation de ces violences. Aucun changement n'est visible dans la zone de comparaison.

1 On a mis ensemble les indécis (ne sait pas) avec ceux qui disent non

2 A l'étude de base et à l'étude intermédiaire, la question n'était pas posée à tout le monde. Elle était adressée à ceux qui reconnaissaient qu'il existe des violences dans la communauté et/ou dans la famille. 


\section{Graphique 6 : Identification en zone expérimentale des changements intervenus au niveau communautaire (étude intermédiaire)}

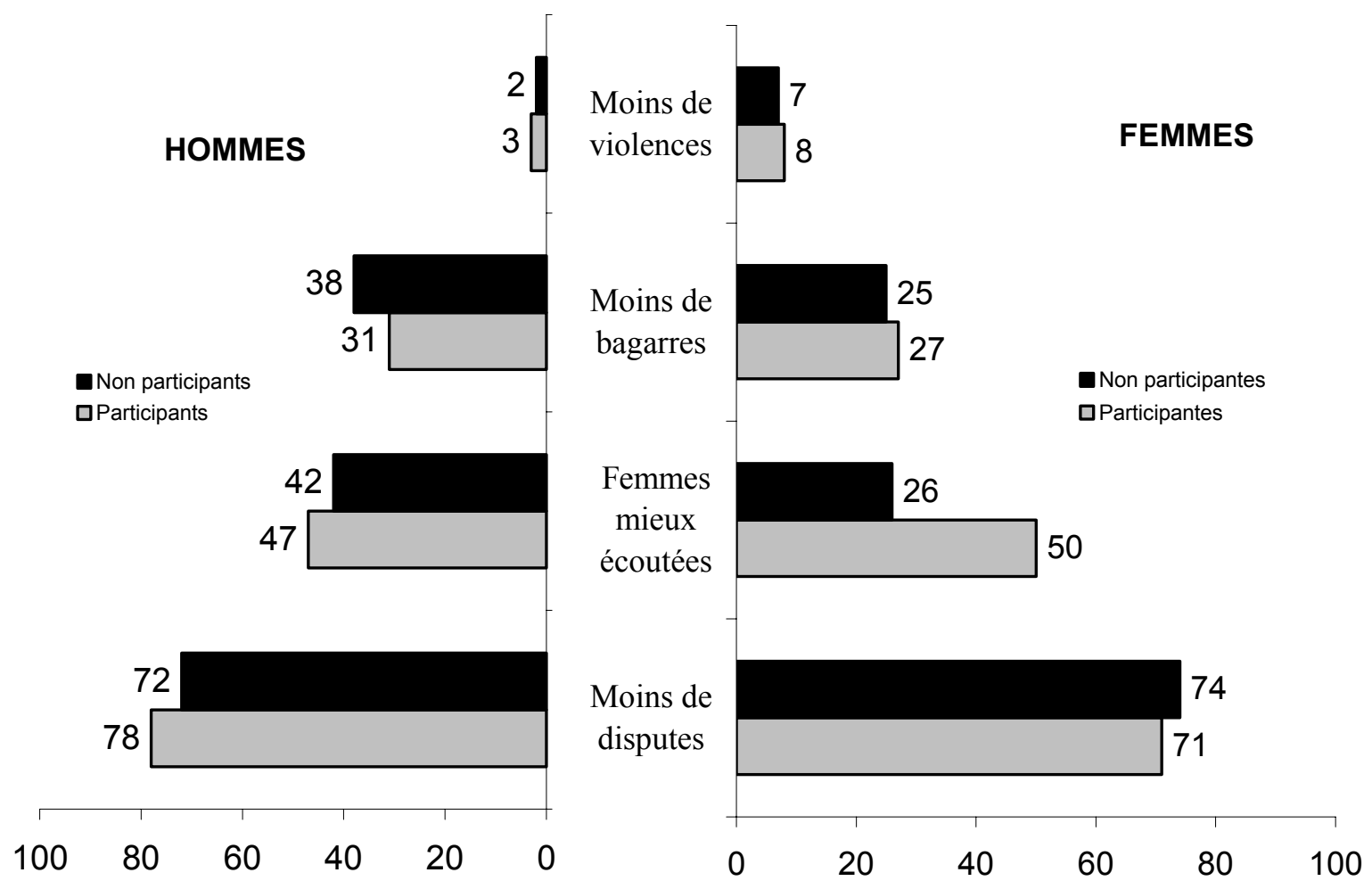

\section{Connaissance sur la santé et Utilisation des services}

La planification familiale, l'hygiène, la lutte contre la diarrhée, le suivi de la grossesse et les IST/VIH/SIDA sont abordés dans cette section. Il faut rappeler que la zone de comparaison était auparavant une zone d'intervention de l'ONG Save The Children et du programme DBC du LSC. L'objectif de ce programme était de mettre à la disposition des formations sanitaires et de leur personnel, des équipements et la formation nécessaires à une plus large diffusion des produits contraceptifs. Les agents de service à base communautaire (ASBC) disposaient également de certains produits contraceptifs et avaient la charge de fournir au niveau village les informations, les conseils indispensables à une meilleure utilisation des contraceptifs. Aujourd'hui, des ONG et associations travaillent toujours dans la zone et apportent aux populations des connaissances, notamment sur la santé.

\section{La planification familiale}

Lorsqu'on examine les connaissances des femmes et des hommes sur les méthodes modernes de contraception, on note une nette différence dès le départ entre les deux zones. La zone de comparaison a des niveaux plus élevés de connaissance que la zone expérimentale. Après l'intervention du programme, le niveau de connaissance des femmes et des hommes participants augmente considérablement (graphique 7). Cependant à l'intérieur de la zone expérimentale, il y a une différence entre les deux groupes de participants et non participants. 
En effet, la connaissance d'une ou deux méthodes de contraception n'a pas changé significativement pour les femmes et les hommes non participants lors de l'évaluation finale. Les courbes des non participants sont presque confondues au fil du temps, signe d'un manque d'amélioration des connaissances, donc de la faiblesse de la diffusion de l'information à travers le parrainage.

\section{Graphique 7 : Connaissance de méthodes modernes de PF en zone expérimentale}

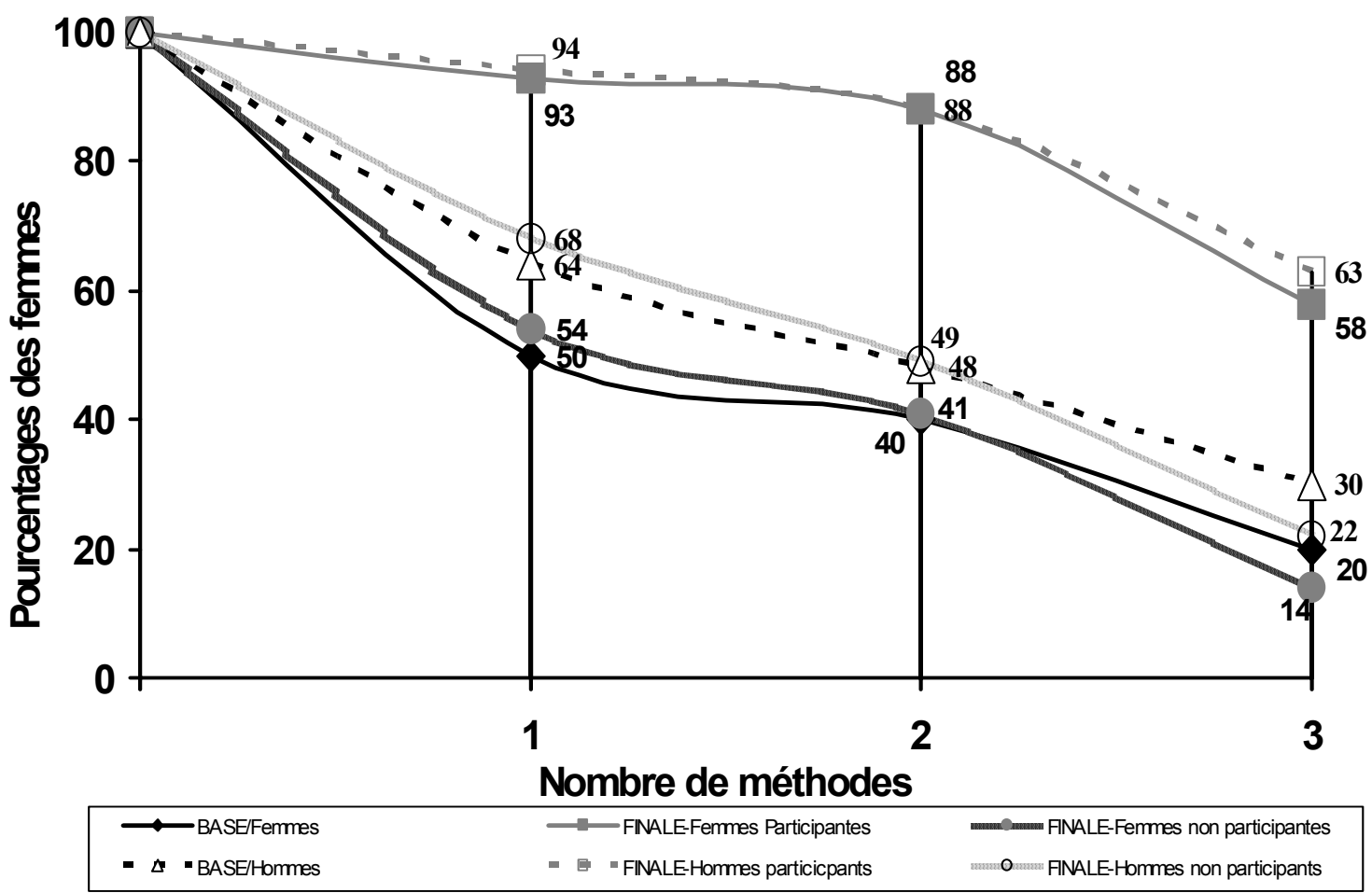

Cependant au tableau 11 on voit que les femmes non participantes avaient augmenté significativement leur connaissance à l'étude intermédiaire. Entre l'étude intermédiaire et l'étude finale, on enregistre une légère baisse aussi bien chez les hommes que chez les femmes, ce qui fait que les niveaux ne sont plus significatifs entre les données de base et l'évaluation finale. Cette baisse est consécutive à l'oubli qui s'installe après le programme, surtout lorsque ces connaissances ne sont pas accompagnées d'utilisation des méthodes. Les moyens modernes de contraception les plus cités sont dans l'ordre: la pilule, l'injectable, le préservatif, l'implant et les spermicides. Les conjoints des femmes qui participent au programme, améliorent également significativement leur niveau de connaissance : de $41 \%$ qui citent deux méthodes modernes à l'étude de base on passe à $62 \%$ après le programme.

En zone de comparaison, on enregistre uniquement chez les femmes une différence significative entre les deux périodes. Mais cette augmentation de l'étude de base à l'étude finale est bien moindre (écart de 13 points) que celle qui a lieu chez les femmes participantes de la zone expérimentale (écart de 48 points). De plus, le test de comparaison des évolutions $(\mathrm{z}=4,80)$ indique que le programme d'intervention a apporté une différence.

Les avantages à espacer les naissances sont reconnus par plus de femmes et d'hommes après le programme (tableau 11). Les avantages les plus cités sont le fait de laisser le bébé grandir 
et de laisser la maman se reposer. Chez les femmes participantes et non participantes et chez les hommes non participants, il y a une baisse significative entre les deux enquêtes post intervention. En ce qui concerne les conjoints des femmes participantes au programme, on note une évolution significative entre l'étude de base (85\%) et l'étude intermédiaire (94\%). Cependant à l'étude finale il y a une baisse significative ( $84 \%)$. En zone de comparaison, il y a une baisse significative chez les hommes alors que le niveau des femmes reste le même.

\section{Tableau 11 : Connaissance et attitude des hommes et des femmes sur la santé de la} reproduction

\begin{tabular}{|c|c|c|c|c|c|c|c|}
\hline \multirow{3}{*}{ Indicateurs } & \multirow[b]{3}{*}{ Base } & \multicolumn{4}{|c|}{ Zone expérimentale } & \multicolumn{2}{|c|}{$\begin{array}{c}\text { Zone de } \\
\text { comparaison }\end{array}$} \\
\hline & & \multicolumn{2}{|c|}{ Intermédiaire } & \multicolumn{2}{|c|}{ Final } & & \\
\hline & & Participants & $\begin{array}{c}\text { Non } \\
\text { participants }\end{array}$ & Participants & $\begin{array}{c}\text { Non } \\
\text { participants }\end{array}$ & Base & Final \\
\hline FEMMES & 662 & 626 & 163 & 578 & 167 & 226 & 228 \\
\hline Connaît au moins deux méthodes modernes de PF & 40 & $91 * *$ & $49 *$ & $* 88 * *$ & 41 & 62 & $75^{* *}$ \\
\hline Il y a des avantages à espacer les naissances & 72 & $98 * *$ & $84 * *$ & $* 95 * *$ & $* * 62 * *$ & 88 & 85 \\
\hline Les 2 époux décident de l'utilisat. de la contraception & 28 & $66^{* *}$ & $42 * *$ & $* * 46 * *$ & $* * 23$ & 31 & 24 \\
\hline Compte utiliser une méthode contraceptive ds l'avenir & 56 & $69 * *$ & 60 & $69 * *$ & 51 & 67 & 60 \\
\hline Le conjoint approuve la contraception & 47 & $80 * *$ & $68 * *$ & $81 * *$ & $* * 51$ & 70 & 67 \\
\hline HOMMES & 697 & 465 & 193 & 448 & 169 & 2227 & 229 \\
\hline Connaît au moins deux méthodes modernes de PF & 48 & $91 * *$ & $65 * *$ & $88^{* * *}$ & **49 & 71 & 67 \\
\hline Il y a des avantages à espacer les naissances & 85 & $98 * *$ & $93 * *$ & $98^{* *}$ & $* * 82$ & 95 & $87 * *$ \\
\hline Les 2 époux décident de l'utilisat. de la contraception & 40 & $71 * *$ & $58 * *$ & $* * 56 * *$ & $49 * *$ & 43 & 38 \\
\hline Compte utiliser une méthode contraceptive ds l'avenir & 67 & $91 * *$ & $77 * *$ & $89 * *$ & 68 & 77 & 70 \\
\hline La conjointe approuve la contraception & 60 & $82 * *$ & $81 * *$ & $84 * *$ & $79 * *$ & 87 & 83 \\
\hline
\end{tabular}

\section{Centre de décision pour l'utilisation de la PF}

A l'étude intermédiaire, la zone expérimentale montre une proportion plus importante de femmes et d'hommes qui citent les deux époux comme centre de décision. Les participants restant proportionnellement plus important à soutenir une telle opinion (tableau 11). En zone de comparaison, aucune différence n'est observable. A l'évaluation finale, ces proportions connaissent de fortes régressions, sauf chez les hommes non-participants qui restent constant dans leur opinion. Chez les conjoints des femmes participantes également, la proportion de ceux qui soutiennent cette assertion augmente avant de baisser lors de l'évaluation finale. Ces baisses résultent en partie de l'importance qui est accordée par les couples au rôle de l'agent de santé. L'agent de santé est perçu comme celui qui décide de l'utilisation ou non de la contraception par l'orientation qu'il peut être amené à faire (examens médicaux, exhortation à utiliser une méthode ou une autre en fonction des résultats des examens, etc.). 


\section{Graphique 8 : Désir d'utilisation future d'une méthode contraceptive}

\section{Zone expérimentale}

\section{Zone de comparaison}

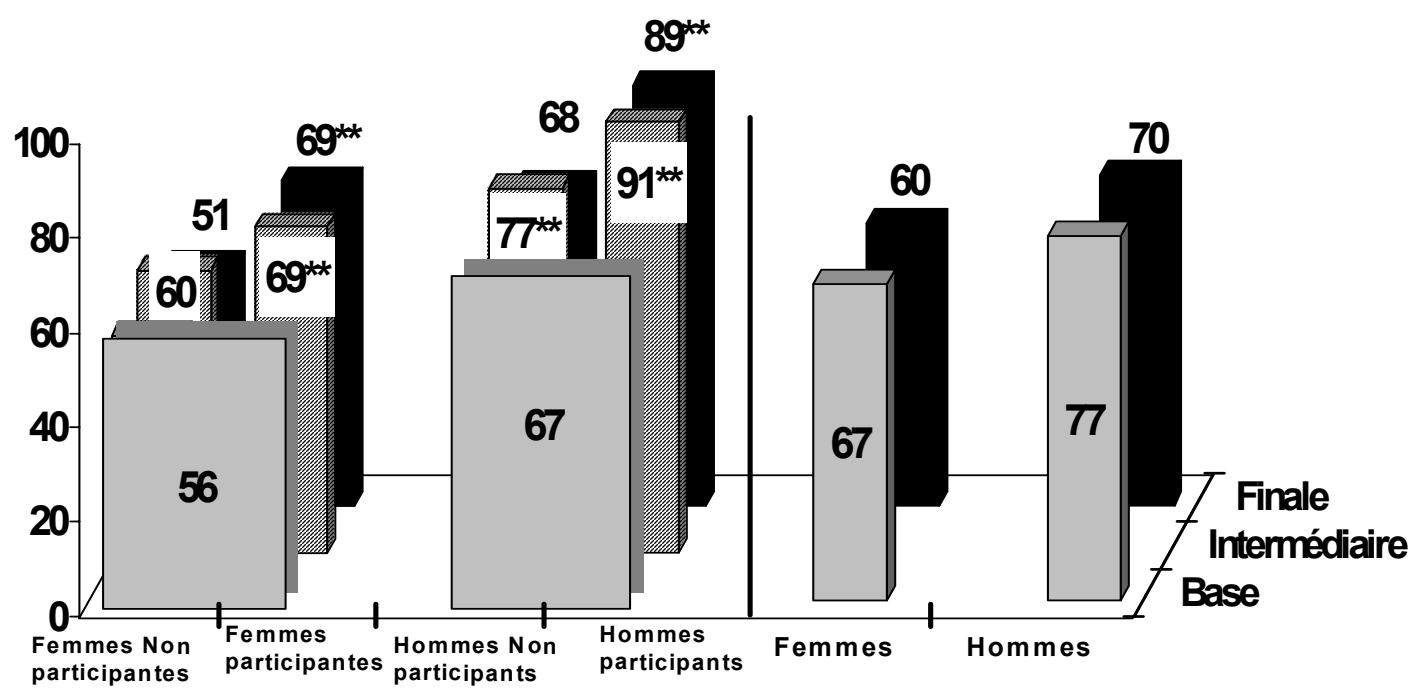

$\square$ Base

$\square$ Intermédiaire

Finale

Dans leur ensemble, les femmes et les hommes participants de la zone expérimentale déclarent être plus disposés à utiliser dans l'avenir une méthode moderne de contraception (graphique 8). A l'étude intermédiaire, les niveaux de connaissance avaient évolués positivement pour les femmes et les hommes participants et pour les hommes non participants. Ces niveaux augmentent significativement encore à l'évaluation finale pour les femmes et les hommes participants. En zone de comparaison, il n'y a aucune différence significative au cours des deux périodes. Les conjoints des participantes par contre sont un peu moins disposés à utiliser la contraception dans le futur passant de 78\% à 66\% (tableau 12).

Tableau 12 : Connaissances des conjoints_des femmes participantes sur la PF et la santé

\begin{tabular}{|c|c|c|c|}
\hline \multirow{2}{*}{ Indicateurs } & \multicolumn{3}{|c|}{ Zone expérimentale } \\
\hline & Base & Intermédiaire & Final \\
\hline PF & 315 & 195 & 281 \\
\hline Avantage à espacer les naissances & 85 & $94 * *$ & $* * 84$ \\
\hline Citent deux méthodes modernes de PF & 41 & $66^{* *}$ & $62 * *$ \\
\hline Les 2 époux doivent utiliser la PF & 38 & $63 * *$ & $* * 48 *$ \\
\hline Compte utiliser une méthode de PF dans le futur & - & 78 & $* * 66$ \\
\hline
\end{tabular}


En résumé, le programme d'éducation semble avoir suscité un besoin de planification familiale à satisfaire dans l'avenir si les réponses données ne sont pas complaisantes. Ce besoin pourrait être comblé par une politique nationale ou sectorielle de distribution à base communautaire des moyens contraceptifs accessibles et à moindres coûts. Ceci est d'autant plus urgent que les participants (hommes et femmes) soutiennent une certaine convergence des opinions dans le couple, notamment en ce qui concerne l'approbation de la contraception moderne. Mis en relation avec le désir d'utilisation future de la contraception, on retiendra que les deux indicateurs évoluent ensemble : les conjoints des femmes (participantes ou non) ne seraient pas favorables à la contraception et ainsi rendent ces femmes moins disposées à utiliser la contraception tandis que chez les autres hommes, les femmes sont beaucoup plus favorables, entraînant ainsi un fort désir d'utilisation de la contraception. Il apparaît ainsi que l'homme reste encore le principal obstacle à l'utilisation de la contraception moderne.

\section{Suivi des grossesses}

Les consultations prénatales (CPN)

Les données du graphique 9 ci-dessous indiquent que dans la zone expérimentale à l'étude intermédiaire, $92 \%$ des femmes participantes et $90 \%$ des non participantes savent qu'il faut au moins trois CPN au cours de la grossesse, soit une augmentation significative des proportions qui au départ étaient de $78 \%$. La proportion des hommes participants n'évolue pas tandis que celle des non participants baisse significativement.

\section{Graphique 9 : Connaissance de trois consultations prénatales}

\section{Zone expérimentale}

\section{Zone de comparaison}

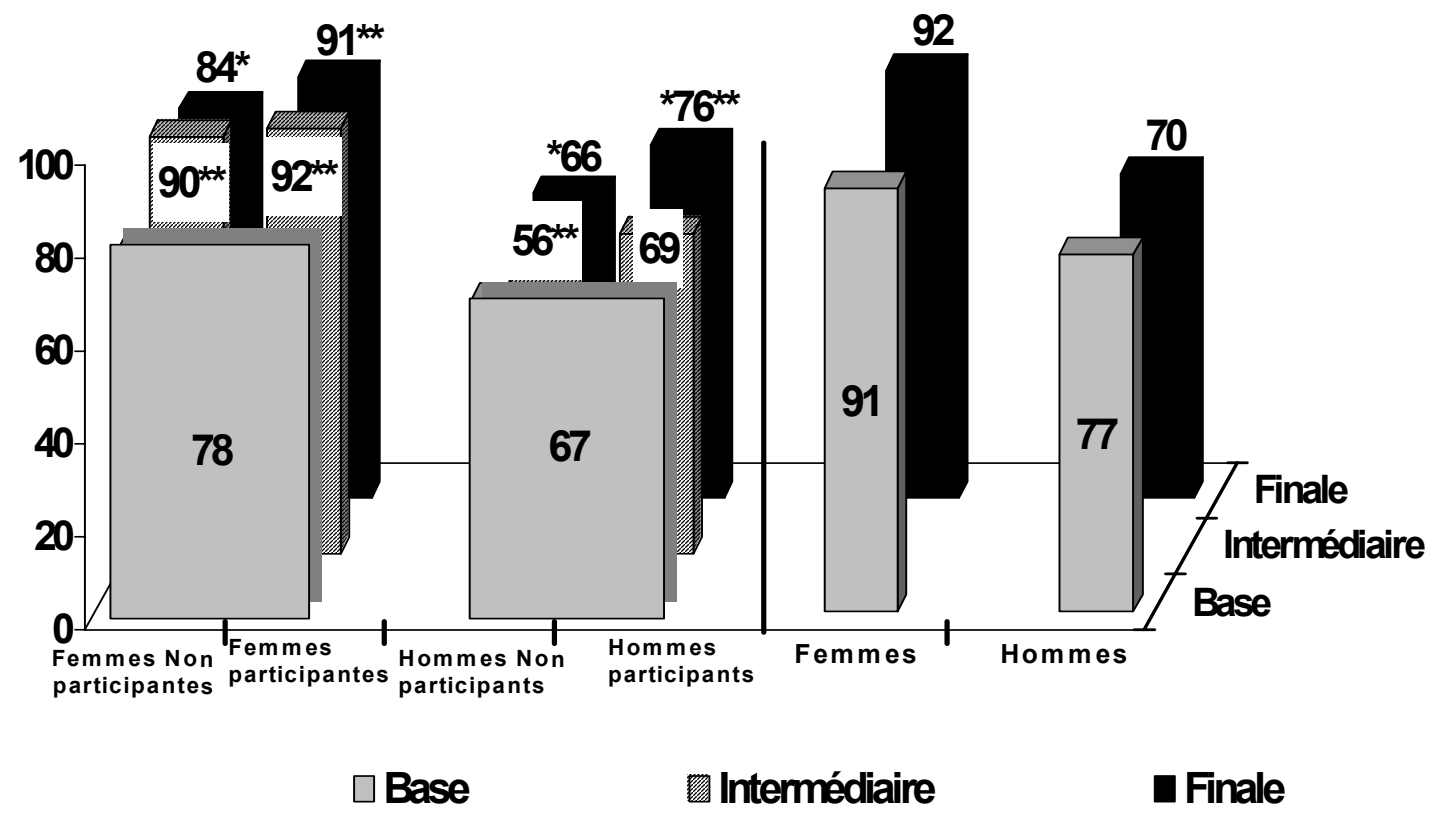

A l'évaluation finale, les niveaux restent aussi élevés chez les femmes participantes, mais ceux des hommes participants augmentent. Il n'y a aucune évolution significative dans la zone de comparaison, aussi bien parmi les hommes que parmi les femmes. Le niveau déjà élevé de la zone de comparaison à l'étude de base s'explique par le rôle que le programme 
santé de la reproduction (SBC) et les différentes actions menées par les ONG et associations ont joué et continuent de jouer sur ce terrain.

Les signes de danger de la grossesse

En ce qui concerne les signes de danger de la grossesse, la proportion des femmes et des hommes de la zone expérimentale qui savent en citer au moins deux est en nette progression $(\mathrm{p}<.00)$. Les signes de danger de la grossesse les plus cités sont les douleurs abdominales, les saignements vaginaux, les fortes poussées de fièvre, l'absence de mouvement du fœtus. A l'étude intermédiaire, la différence de niveau est significative chez les participants. En effet, $28 \%$ des femmes participantes et $17 \%$ des hommes participants savent citer au moins deux de ces signes, contre seulement $4 \%$ avant la mise en œuvre du programme (graphique 10). Chez les hommes, les non participants n'ont pas amélioré leurs connaissances.

\section{Graphique 10 : Cite au moins deux signes de danger de la grossesse}

\section{Zone expérimentale}

\section{Zone de comparaison}

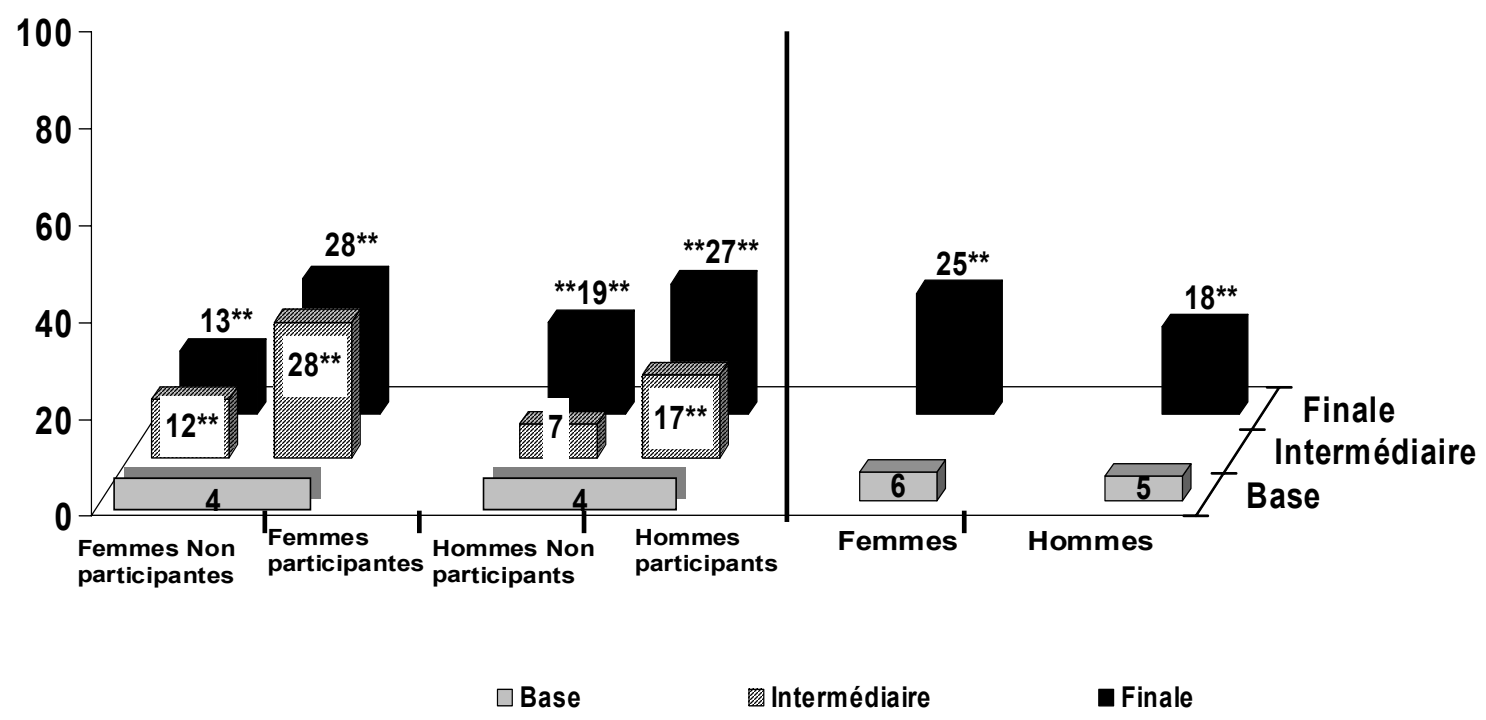

A l'évaluation finale les niveaux restent les mêmes chez les femmes et augmentent chez les hommes $(\mathrm{P}<.00)$. En zone de comparaison, le niveau de connaissance a significativement évolué aussi bien chez les hommes que chez les femmes. Le test de comparaison des évolutions permet de signaler que l'évolution de la zone expérimentale pour les hommes est plus importante que celle de la zone de comparaison $(z=2,17)$. Pour les femmes il n'y a pas de différence entre les deux zones. 


\section{La survie de l'enfant}

\section{Graphique 11: Cite les bonnes habitudes alimentaires contre la diarrhée}

\section{Zone expérimentale}

\section{Zone de comparaison}

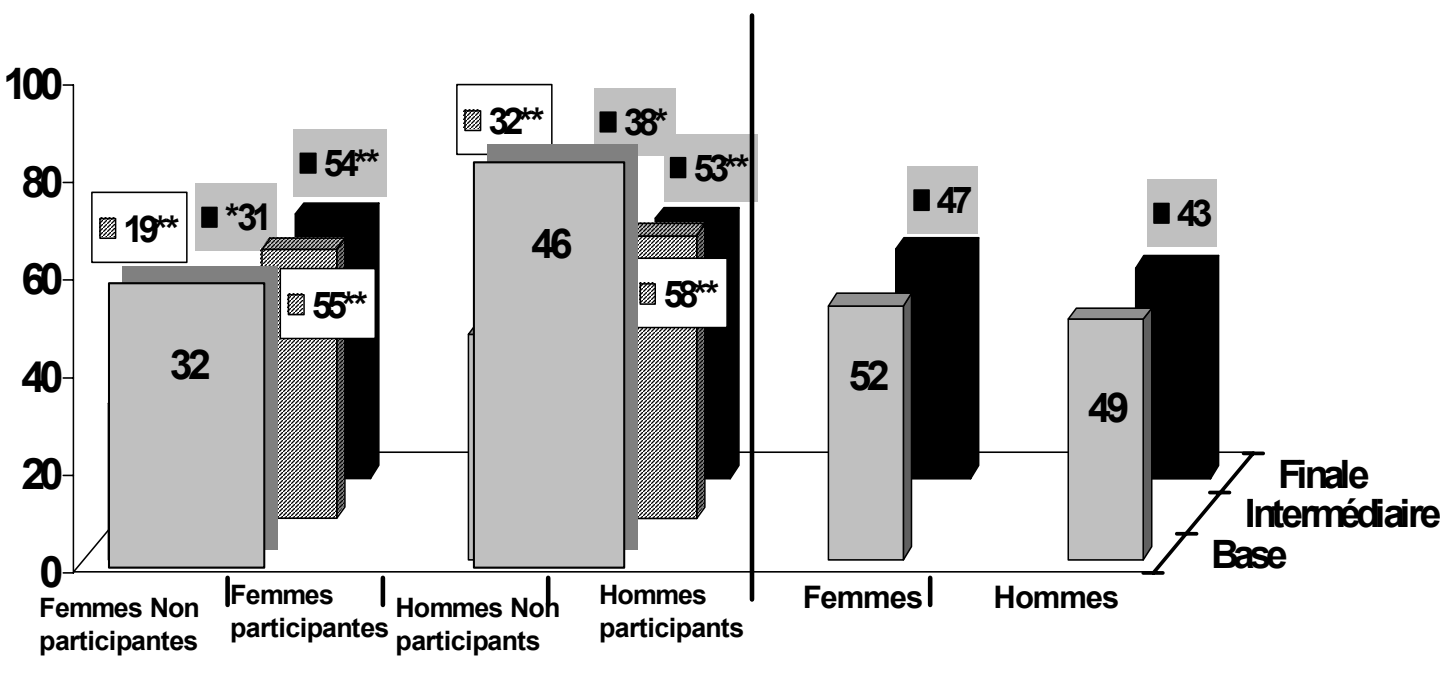

Base

$\square$ Intermédiaire

Finale

La diarrhée est l'une des principales causes de la mortalité infanto-juvénile. Elle a pour cela une place importante dans le module hygiène et prévention de la maladie. Les données du graphique 11 indiquent à l'étude intermédiaire que la proportion de participants (hommes et femmes) de la zone expérimentale qui ont une bonne connaissance de la question, augmente significativement. Parmi les non participants, il y a une baisse significative $(p<.01)$ à l'étude intermédiaire, due au biais de sélection. A l'étude finale, le niveau des femmes participantes reste la même mais elle augmente $(\mathrm{p}<.05)$ chez les non participantes, de sorte à ce que le niveau s'équilibre désormais avec le niveau de départ. Chez les hommes, il n'y a d'évolution significative aussi bien chez les participants que parmi les non participants. En zone de comparaison, aucune différence significative n'est observée entre les hommes et les femmes au cours de la période.

\section{Les IST/VIH/SIDA}

\section{La séropositivité}

De façon générale, le VIH est plus connu que les IST. Lorsqu'on interroge les hommes et les femmes ayant participé au programme d'éducation sur la notion de séropositivité, on constate que la proportion de ceux qui soutiennent qu'une personne apparemment en bonne santé peut être en réalité atteinte du virus est beaucoup plus élevée. A l'étude intermédiaire, seuls les participants (hommes et femmes) avaient augmenté leurs connaissances de manière significative (tableau 13). Mais à l'évaluation finale, la différence est significative pour tous les sous groupes (femmes, hommes, conjoints des participantes). Il y a eu une légère 
augmentation ( $p>.05)$, entraînant pour les non participants un niveau différent de celui de l'étude de base. Dans la zone de comparaison, les niveaux de connaissance au départ étaient plus élevés que dans la zone expérimentale. Que ce soit chez les femmes comme chez les hommes, aucune différence n'est perceptible au cours de la période. Aussi on peut conclure qu'au-delà de la campagne menée à travers tout le pays sur le SIDA ainsi que le programme d'éducation a contribué à améliorer les connaissances des habitants de la zone expérimentale sur la notion de séropositivité.

Tableau 13 : Niveau de connaissance et attitudes des hommes et des femmes sur les IST/ VIH/SIDA

\begin{tabular}{|c|c|c|c|c|c|c|c|}
\hline \multirow{3}{*}{ Indicateurs } & \multicolumn{5}{|c|}{ Zone expérimentale } & \multicolumn{2}{|c|}{$\begin{array}{c}\text { Zone de } \\
\text { comparaison }\end{array}$} \\
\hline & \multirow[b]{2}{*}{ Base } & \multicolumn{2}{|c|}{ Intermédiaire } & \multicolumn{2}{|c|}{ Final } & \multirow[b]{2}{*}{ Base } & \multirow[b]{2}{*}{ Final } \\
\hline & & Participants & $\begin{array}{c}\text { Non } \\
\text { participants }\end{array}$ & Participants & $\begin{array}{c}\text { Non } \\
\text { participants } \\
\end{array}$ & & \\
\hline FEMMES & 662 & 626 & 163 & 578 & 167 & 226 & 228 \\
\hline $\begin{array}{l}\text { Une personne en bonne santé peut être porteuse du } \\
\text { VIH/SIDA }\end{array}$ & 47 & $82 * *$ & 52 & $86^{* *}$ & $61 * *$ & 65 & 67 \\
\hline $\begin{array}{l}\text { Cite au moins deux moyens de prévention contre } \\
\text { les IST/VIH/ SIDA }\end{array}$ & 37 & $63^{* *}$ & 29 & $59 * *$ & 38 & 51 & 52 \\
\hline HOMMES & 697 & 465 & 193 & 448 & 169 & 227 & 229 \\
\hline $\begin{array}{l}\text { Une personne en bonne santé peut être porteuse du } \\
\text { VIH/SIDA }\end{array}$ & 71 & $89^{* *}$ & 75 & $92 * *$ & $79 * *$ & 76 & 76 \\
\hline $\begin{array}{l}\text { Cite au moins deux moyens de prévention contre } \\
\text { lesIST/VIH/ SIDA }\end{array}$ & 66 & $78^{* *}$ & 62 & $76^{* *}$ & 63 & 74 & $65^{* *}$ \\
\hline
\end{tabular}

\section{Les moyens de prévention contre les IST et le VIH/SIDA}

Les moyens de prévention les plus cités sont : avoir un seul partenaire sexuel, utiliser le préservatif, l'abstinence sexuelle et le fait d'éviter d'avoir des rapports sexuels avec des prostituées. Il y a eu une augmentation sensible de la proportion des participants connaissant ces moyens de prévention (tableau 13), contrairement aux non participants. A l'évaluation finale, les niveaux sont restés les mêmes pour tous les groupes. En zone de comparaison, il n'y pas de changement chez les femmes tandis que le niveau de connaissance des hommes, préalablement plus élevé que celui des hommes de la zone expérimentale, connaît une baisse importante. Par conséquent, on peut conclure que le programme a contribué à améliorer la connaissance des moyens de prévention contre les IST/VIH/SIDA.

En conclusion de la section sur la santé on peut dire que le niveau de connaissance des participants a augmenté de façon significative pour tous les indicateurs: les méthodes modernes de PF, les avantages de la PF, le centre de décision en matière de PF, le suivi prénatal, les dangers de la grossesse, les bonnes habitudes alimentaires contre la diarrhée, la séropositivité et les moyens de prévention contre les IST/VIH/SIDA. Il en est de même pour les attitudes positives telles que l'approbation et l'utilisation future de la contraception. Par contre, on enregistre chez les femmes non participantes une évolution significative uniquement pour les consultations prénatales, les dangers de la grossesse et la notion de séropositivité. Parmi les hommes non participants, il s'agit uniquement du centre de décision dans le couple en matière de contraception, l'approbation de la conjointe pour l'utilisation de la contraception, les dangers de la grossesse et la notion de séropositivité. En zone de comparaison, seulement deux indicateurs ont observé chez les femmes une évolution significative de connaissance : les méthodes modernes de PF et les dangers de la grossesse. Chez les hommes, il s'agit uniquement des dangers de la grossesse. 


\section{L'excision}

\section{Connaissances sur l'excision}

\section{La nécessité de la pratique de l'excision}

Les données montrent au départ des niveaux très élevés. La grande majorité des personnes pensent que l'excision n'est pas une pratique nécessaire (graphique 12). Après le programme, le groupe d'intervention montre une augmentation significative. Chez les non-participantes et les hommes, l'opinion est restée la même au cours des deux enquêtes post intervention. Les conjoints des participantes (tableau 15 plus bas) montrent la même tendance. Entre l'étude de base et l'évaluation finale, l'indicateur est passé de $79 \%$ à $90 \%$. Dans la zone de comparaison, la proportion de femmes qui disent que la pratique n'est pas nécessaire augmente $(\mathrm{p}<.05)$, tandis que la proportion des hommes baisse $(\mathrm{p}<.05)$. Le test de comparaison des évolutions entre les femmes des deux zones ne montre pas d'effet supplémentaire dans la zone expérimentale.

\section{Graphique 12 : L'excision n'est pas une pratique nécessaire}

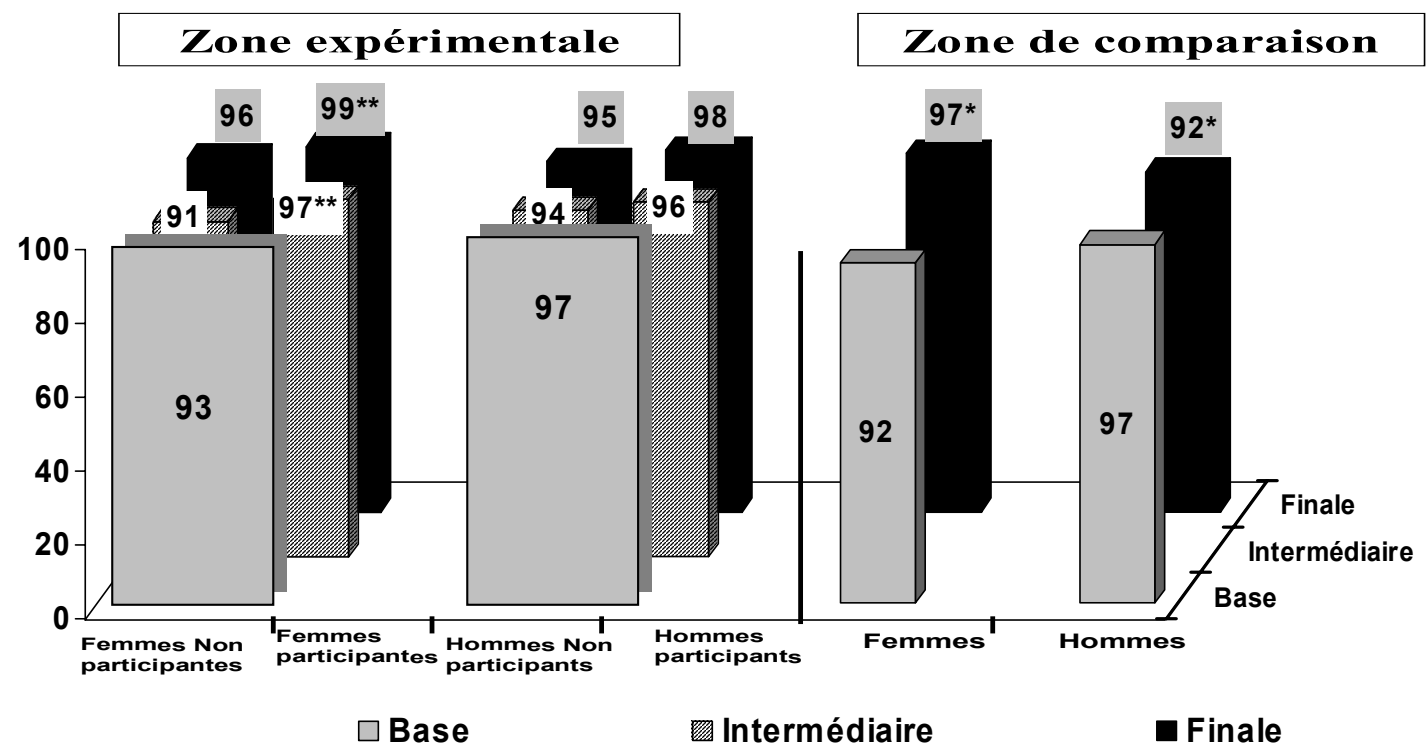

Les conséquences néfastes de l'excision

La connaissance des effets néfastes de l'excision, très forte déjà au départ, augmente significativement chez les femmes $(82 \%$ à $97 \%)$ et les hommes ( $85 \%$ à $97 \%)$ participants au programme dès l'étude intermédiaire. Cependant, on n'observe aucune augmentation de la connaissance chez les femmes et les hommes non-participants ( $81 \%$ et $86 \%)$. A l'étude finale, les tendances se maintiennent, sauf pour les hommes non participants où on assiste à une forte baisse $(\mathrm{p}<.05)$, rendant les proportions plus faibles que celles de l'étude de base. En zone de comparaison, aucune augmentation des connaissances n'est observée chez les femmes mais une baisse significative $(\mathrm{p}<.01)$ est constatée chez les hommes. Les changements ainsi constatés en zone expérimentale parmi les participants peuvent être attribués au programme.

Lorsqu'il s'agit de citer au moins deux dangers de l'excision, on note à l'étude intermédiaire une évolution significative chez les participants hommes et femmes (graphique 13). Si au départ les femmes (52\%) étaient moins informées que les hommes (64\%), à l'étude intermédiaire, la proportion des femmes participantes est plus importante (87\%) que celle des 
hommes participants (83\%). Dans le même temps, on ne note aucune progression chez les femmes et les hommes non-participants.

\section{Graphique 13 : Cite au moins deux dangers de l'excision}

\section{Zone expérim entale}

\section{Zone de comparaison}

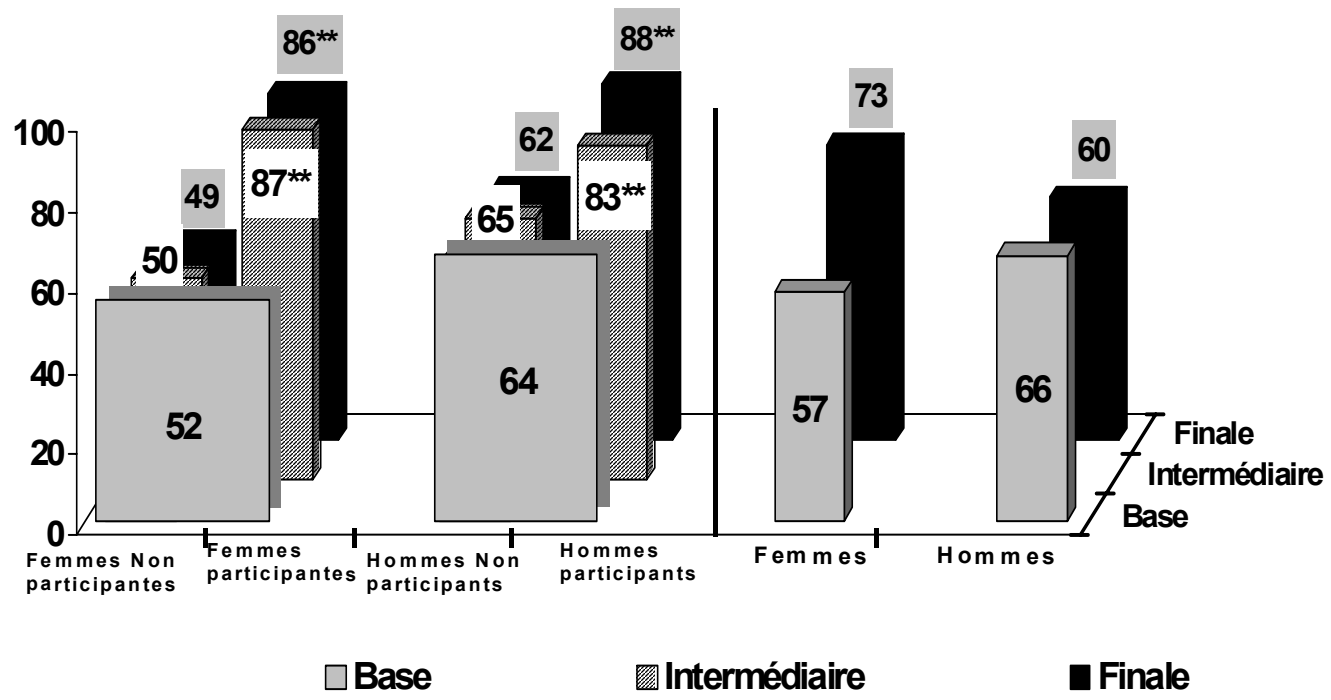

Un an après la mise en œuvre du programme, l'étude finale révèle que les femmes et les hommes participants au programme ont conservé le même niveau de connaissance. Aucun changement n'est aussi observé chez les non-participants. Quant aux conjoints des participantes, ils n'ont pas amélioré leur connaissance. Une situation qui traduirait ainsi l'absence de transfert de l'information au sein du ménage ou une sous-information.

En zone de comparaison, aucune évolution du niveau de connaissance n'est observable. Par conséquent, cette évolution parmi les participants peut être attribuée en grande partie au programme d'éducation.

\section{Attitudes sur l'excision}

L'excision comme atteinte aux droits de la femme

A l'évaluation finale, 9 participants sur 10 (hommes et femmes) estiment que l'excision est une atteinte aux droits de la femme (tableau 14). Chez les non-participants, les niveaux sont plus bas, et les hommes ont une meilleure attitude que les femmes. Les conjoints des femmes ayant participé au programme déclarent en plus grand nombre à l'évaluation finale que l'excision est une atteinte aux droits de la femme (voir tableau 15 plus bas). 


\section{Désapprobation de l'excision}

C'est surtout parmi les femmes et les hommes participants que la désapprobation de l'excision est la plus forte au fil du temps. Avec un niveau déjà élevé à l'étude de base $(89 \%)$, la proportion d'hommes et de femmes participants a connu une évolution significative, surtout chez les hommes (graphique 14). Aucune évolution n'est observée chez les non-participantes. A l'étude finale, la différence se maintient. Cette différence est accentuée chez les femmes au cours de la dernière période $(\mathrm{p}<.01)$, contrairement aux hommes qui n'ont observé aucun changement sensible. La désapprobation de l'excision par les conjoints est très élevée et constante sur la période (tableau 15). En zone de comparaison, aucun changement n'est observé aussi bien chez les femmes que chez les hommes.

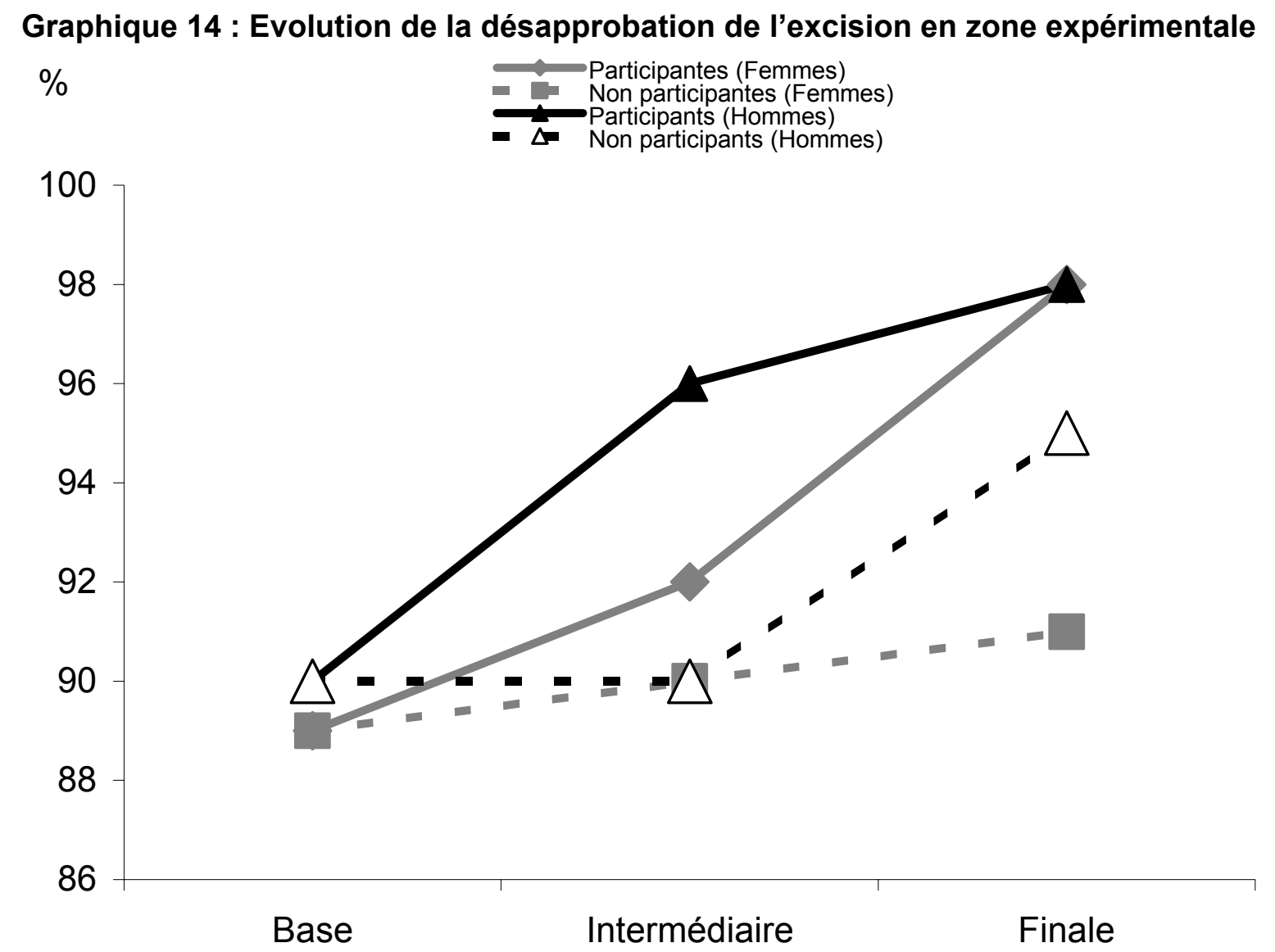

Tous les partenaires des enquêtés de la zone expérimentale désapprouvent l'excision selon les déclarations du conjoint. Par contre en zone de comparaison, la proportion d'hommes qui déclarent que leur partenaire désapprouve l'excision baisse significativement (tableau 14). 
Tableau 14 : Attitude des hommes et des femmes sur l'excision

\begin{tabular}{|c|c|c|c|c|c|c|c|}
\hline \multirow{3}{*}{ Indicateurs } & \multicolumn{5}{|c|}{ Zone expérimentale } & \multicolumn{2}{|c|}{ Zone de comparaison } \\
\hline & \multirow[b]{2}{*}{ Base } & \multicolumn{2}{|c|}{ Intermédiaire } & \multicolumn{2}{|c|}{ Final } & \multirow[b]{2}{*}{ Base } & \multirow[b]{2}{*}{ Final } \\
\hline & & Participants & $\begin{array}{c}\text { Non } \\
\text { participants }\end{array}$ & Participants & $\begin{array}{c}\text { Non } \\
\text { participants }\end{array}$ & & \\
\hline FEMMES (N) & 662 & 626 & 163 & 578 & 167 & 226 & 228 \\
\hline Désapprouve l'excision ${ }^{1}$ & 89 & $92 *$ & 90 & $* * 98 * *$ & 91 & 90 & 94 \\
\hline Partenaire désapprouve l'excision & 97 & 99 & 95 & $99^{2} *$ & 98 & 97 & 96 \\
\hline Excision atteinte aux droits de la femme & - & 91 & 56 & $* 94$ & 64 & - & 66 \\
\hline Compte exciser sa fille & $3^{3}$ & $1 * *$ & 6 & $1 * *$ & 2 & 4 & 2 \\
\hline Regret d'avoir exciser sa fille & $\begin{array}{c}53 \\
(n=119)\end{array}$ & $\begin{array}{c}69 * * \\
(n=137)\end{array}$ & $\begin{array}{c}64 \\
(n=45)\end{array}$ & $\begin{array}{c}* 81 * * \\
(n=134)\end{array}$ & $\begin{array}{c}67 \\
(n=33)\end{array}$ & $\begin{array}{c}50 \\
(n=52)\end{array}$ & $\begin{array}{c}64 \\
(n=56)\end{array}$ \\
\hline HOMMES (N) & 697 & 465 & 193 & 448 & 169 & 227 & 229 \\
\hline Désapprouve l'excision ${ }^{4}$ & 90 & $96^{* *}$ & 90 & $98^{* *}$ & *95* & 93 & 89 \\
\hline Partenaire désapprouve l'excision & 98 & 99 & 97 & 97 & 96 & 99 & $89 * *$ \\
\hline Excision atteinte aux droits de la femme & - & 91 & 70 & $* 96$ & 72 & - & 73 \\
\hline Compte exciser sa fille & 3 & $1 *$ & 1 & 2 & 3 & 2 & $7 *$ \\
\hline
\end{tabular}

\section{Durée de désapprobation de l'excision}

En majorité la désapprobation de l'excision est antérieure au programme ${ }^{5}$. Dans la zone de comparaison et parmi les non participants de la zone expérimentale, environ 8 femmes et hommes sur 10 désapprouvent l'excision depuis plus de 2 ans, alors que parmi les participants hommes et femmes cela touche environ 6 personnes sur 10. Les niveaux de désapprobation qui remontent avant le programme sont dus aux fortes campagnes de sensibilisation menée par le comité nationale de lutte contre la pratique de l'excision (CNLPE) mais également par la répression des contrevenants à la loi. On note que un tiers des participants (36\% des femmes et 37\% des hommes) désapprouvent l'excision depuis seulement deux ans, c'est-à-dire depuis l'arrivée du programme. Il y a aussi $16 \%$ des femmes et $12 \%$ des hommes non-participants qui attribuent leur désapprobation au programme de Mwangaza.

\section{Regret et désir futur d'excision}

Dans l'ensemble, très peu de femmes et d'hommes ont l'intention d'exciser leurs filles qui ne le sont pas encore ( $3 \%$ à l'enquête de base). Une baisse significative est néanmoins observée chez les femmes et les hommes participants (1\%). En zone de comparaison, il n'y a aucun changement sauf chez les hommes (tableau 14). Les conjoints également sont très peu nombreux à vouloir exciser leur fille (3\% à l'évaluation finale).

La question sur le sentiment de regret de l'excision des filles a été posée uniquement aux femmes ayant au moins une fille excisée. Parmi cet échantillon des femmes ayant eu au moins une fille excisée, environ la moitié regrette d'avoir excisé sa fille au départ. On note une augmentation significative uniquement chez les participantes à l'enquête intermédiaire. Un an après le programme, le nombre de femmes qui regrette l'excision de leur fille augmente considérablement chez les participantes $(\mathrm{p}<.05)$. Chez les non-participantes et en zone de comparaison, aucune différence significative n'est observée au fil du temps, malgré l'augmentation des proportions (tableau 14).

\footnotetext{
${ }^{1}$ Ces proportions comprennent les indécises

${ }^{2}$ Il existe des femmes célibataires dans cet effectif.

${ }^{3}$ A l'étude de base, la question a été posée uniquement aux femmes qui approuvent l'excision, les autres étant considérées comme n'ayant pas l'intention d'exciser leur fille

${ }^{4}$ Ces proportions comprennent les indécis

${ }^{5}$ Il a été demandé aux enquêtés de préciser la période depuis laquelle ils n'approuvent pas l'excision
} 
Tableau 15 : Connaissances et Attitude des conjoints des femmes participantes sur l'excision

\begin{tabular}{|c|c|c|c|}
\hline \multirow{2}{*}{ Indicateurs } & \multicolumn{3}{|c|}{ Zone expérimentale } \\
\hline & Base & Intermédiaire & Final \\
\hline EXCISION & 315 & 195 & 281 \\
\hline "L'excision n'est pas une pratique nécessaire ${ }^{I}$ & 79 & 85 & $90 * *$ \\
\hline Cite au moins deux conséquences néfastes de l'excision & 58 & 62 & 66 \\
\hline Discute de l'excision avec les autres & 59 & 65 & $* * 85 * *$ \\
\hline Connaissance de la loi contre l'excision & 98 & $86 * *$ & $* * 100 * *$ \\
\hline Désapprouve l'excision ${ }^{2}$ & $96^{3}$ & 93 & 95 \\
\hline Partenaire désapprouve l'excision & 99 & 98 & 99 \\
\hline Excision atteinte aux droits de la femme & 1 & $66^{* *}$ & $* * 77 * *$ \\
\hline Compte exciser sa fille ${ }^{4}$ & 1 & 2 & 3 \\
\hline
\end{tabular}

\section{Prévalence de l'excision}

\section{L'Etat actuel de l'excision dans les deux zones}

Au cours de l'évaluation finale, on a enregistré 1760 filles en vie (464 en zone de comparaison et 1296 en zone expérimentale) parmi lesquelles $22 \%$ sont excisées. Le rang de naissance va de 1 à 8 et les âges varient de zéro à plus de 20 ans, la moitié des filles $(52 \%)$ étant âgée de 0 à 10 ans révolus. Parmi ces filles, celles qui sont excisées représentent au total $3 \%$. L'état d'excision des filles est obtenu de façon indirecte, c'est-à-dire sur déclaration de leur mère ${ }^{5}$. Le tableau 16 suivant donne la répartition des filles excisées selon les groupes d'âges.

Tableau 16 : Etat d'excision (en \%) des filles en vie

\begin{tabular}{|c|c|c|c|c|c|c|c|}
\hline & \multicolumn{5}{|c|}{ Zone expérimentale } & \multicolumn{2}{|c|}{ Zone de comparaison } \\
\hline & \multirow[b]{2}{*}{ BASE } & \multicolumn{2}{|c|}{ INTERMÉDIAIRE } & \multicolumn{2}{|c|}{ FINAL } & \multirow[b]{2}{*}{ BASE } & \multirow[b]{2}{*}{ FINAL } \\
\hline & & Participantes & $\begin{array}{c}\text { Non } \\
\text { Participantes } \\
\end{array}$ & Participantes & $\begin{array}{c}\text { Non } \\
\text { Participantes }\end{array}$ & & \\
\hline Total filles en vie & $\begin{array}{c}24 \\
(942)\end{array}$ & $\begin{array}{c}27 \\
(967)\end{array}$ & $\begin{array}{l}30^{*} \\
(294)\end{array}$ & $\begin{array}{c}25 \\
(975)\end{array}$ & $\begin{array}{l}* * 21 \\
(321)\end{array}$ & $\begin{array}{c}22 \\
(455)\end{array}$ & $\begin{array}{c}17 \\
(464)\end{array}$ \\
\hline $\begin{array}{l}\text { Prévalence réelle de l'excision } \\
\text { (filles } \geq 9 \text { ans) }\end{array}$ & $\begin{array}{c}43 \\
(485)\end{array}$ & $\begin{array}{c}47 \\
(529)\end{array}$ & $\begin{array}{c}50 \\
(169)\end{array}$ & $\begin{array}{c}45 \\
(530)\end{array}$ & $\begin{array}{c}* 38 \\
(175)\end{array}$ & $\begin{array}{c}38 \\
(239)\end{array}$ & $\begin{array}{l}25^{* *} \\
(294)\end{array}$ \\
\hline Filles de $0-4$ ans $^{6}$ & $\begin{array}{c}1 \\
(257)\end{array}$ & $\begin{array}{c}0 \\
(237)\end{array}$ & $\begin{array}{c}1 \\
(71)\end{array}$ & $\begin{array}{c}0 \\
(224)\end{array}$ & $\begin{array}{c}0 \\
(82)\end{array}$ & $\begin{array}{c}0 \\
(110)\end{array}$ & $\begin{array}{l}3 \\
(74)\end{array}$ \\
\hline Filles de 5-9 ans & $\begin{array}{c}9 \\
(246)\end{array}$ & $\begin{array}{c}11 \\
(242)\end{array}$ & $\begin{array}{c}10 \\
(70)\end{array}$ & $\begin{array}{c}* 5 \\
(261)\end{array}$ & $\begin{array}{c}3 \\
(77)\end{array}$ & $\begin{array}{c}6 \\
(127)\end{array}$ & $\begin{array}{c}3 \\
(123)\end{array}$ \\
\hline Filles de $0-10$ ans & $\begin{array}{c}6 \\
(556)\end{array}$ & $\begin{array}{c}7 \\
(525)\end{array}$ & $\begin{array}{c}10 \\
(154)\end{array}$ & $\begin{array}{l}* * 3 * \\
(519)\end{array}$ & $\begin{array}{l}* * 1 * \\
(171)\end{array}$ & $\begin{array}{c}4 \\
(260)\end{array}$ & $\begin{array}{c}4 \\
(217)\end{array}$ \\
\hline
\end{tabular}

NB: Les chiffres entre parenthèses représentent l'effectif total des filles pour chaque groupe d'âges

Les différences significatives entre périodes dans chaque zone s'expriment ainsi: ${ }^{*} p<0,05 * * p<0,01$

\footnotetext{
${ }^{1}$ La question n'ayant pas été posée à l'étude de base, on a considéré ceux qui disent que l'excision comporte des effets néfastes

${ }^{2}$ Ces proportions ne comprennent pas les indécises

${ }^{3}$ A l'étude de base, on a considéré ceux qui disent qu'il y a des avantages à ne pas exciser les filles

${ }^{4}$ La question a été posée à l'étude de base à ceux qui ont des filles non excisées.

${ }^{5}$ Toutes les autres collectes des informations ont procédé de la même manière

${ }^{6}$ Les âges sont en années révolues. Cette répartition tient compte des âges quinquennaux dans la plupart des études et permet ainsi des comparaisons. Le groupe des filles de 0-10 ans constitue la population cible des filles dans le cadre de cette étude
} 
La prévalence réelle de l'excision chez les filles ne peut être obtenue que lorsque celles qui courent encore le risque de subir la pratique sont exclues de l'analyse ${ }^{1}$. L'âge moyen à l'excision dans la zone du LSC était en 1998 de 8 ans (LSC N²1, 1998) tandis que l'EDS estime à $54 \%$ la proportion des filles excisées avant cet âge. Dans notre échantillon de filles, l'âge médian à l'excision est de 5 ans, quelle que soit la zone. En excluant toutes les filles en vie de plus de 8 ans $^{2}$, on obtient à l'étude finale une prévalence réelle de $43 \%(\mathrm{n}=705)$ en zone expérimentale et de $25 \%(\mathrm{n}=294)$ en zone de comparaison. Bien qu'on ait observé à l'étude de base une baisse de la pratique de l'excision de l'ordre de $43 \%$, on a également observé qu'en comparant avec l'âge à l'excision des mères, le calendrier de l'excision avait changé avec un âge à l'excision en baisse pour les filles.

A l'étude intermédiaire on note que plus de mères non participantes ont déclaré que leurs filles étaient excisées (de 24\% à 30\%). Aucune évolution n'est observée chez les femmes participantes, ni à l'évaluation finale. Par contre on voit une baisse significative pour les filles des femmes non participantes, de sorte que le niveau redevient comparable à celui de départ. En zone de comparaison, il n'y a pas de changement sensible au fil du temps (tableau 16). La prévalence de l'excision montre une évolution identique, sauf pour les filles des femmes non participantes chez qui on enregistre une baisse significative entre les niveaux de l'étude intermédiaire (38\%) et celui de l'évaluation finale (50\%). Mais il n'y a pas de différences significatives avec le niveau de départ (43\%). La zone de comparaison montre par contre une baisse significative entre les deux périodes. Il faut rappeler que la zone de comparaison est peuplée en majorité de chrétiens contrairement à la zone d'intervention à majorité musulmane. La prévalence de l'excision étant plus basse dans la population chrétienne selon l'EDS 1999, il se peut que le déclin de l'excision soit plus rapide dans ce groupe.

La situation des filles de 0-10 ans dans la zone expérimentale montre que si aucune évolution n'est observable entre l'étude de base et l'étude intermédiaire, à l'évaluation finale on assiste à une baisse significative des proportions de filles excisées chez toutes les femmes, de sorte que le niveau devient significativement différent $(\mathrm{p}<.05)$ de celui de l'étude de base. En zone de comparaison, aucune différence significative n'est observée chez les filles de 0-10 ans (tableau 16).

Dans la zone d'intervention, la grande majorité des femmes et des hommes n'ont plus l'intention d'exciser leurs filles, ce qui demeure cohérent avec la baisse constatée du niveau de l'excision au sein des petites filles. Au regard des actions antérieures entreprises dans le cadre du LSC, des activités du CNLPE et des activités récentes, les populations devraient désormais être dans un processus de changement social dont la déclaration publique peut être considérée comme un mécanisme de renforcement et de consolidation des capacités.

De l'enquête de base à l'enquête finale, on note une fluctuation du niveau général d'excision, dû à un effet d'échantillonnage ou de déclarations. Au départ, $24 \%$ de l'ensemble des filles en vie étaient excisées. A l'étude intermédiaire, elles représentaient $28 \%$ contre $22 \%$ à l'étude finale. Notons que dans la zone d'intervention, aucune fille de moins de 5 ans n'est excisée, que la mère ait participé ou non au programme. A l'étude intermédiaire dans la même zone, on avait pu noter une situation identique, tandis que $1 \%$ des enfants de cet âge avaient été excisées à l'étude de base (tableau 16). S'il est vrai que les filles de la zone expérimentale

\footnotetext{
${ }^{1}$ Voir Enquête Démographique et de Santé.

${ }^{2}$ Ce qui donne la prévalence réelle de l'excision
} 
n'ont pas encore dépassé l'âge moyen à l'excision (5,7 ans), on peut penser qu'il y a un abandon de la pratique dans la province. Ceci est d'autant plus vrai que l'excision se pratique de plus en plus aux âges très jeunes. C'est en zone de comparaison qu'on enregistre $3 \%$ de filles de moins de 5 ans excisées. Comparée aux données de l'étude de base où aucune fille n'était excisée, il y a comme une persistance de la pratique au sein d'un petit groupe dans cette zone.

\section{Table de survie des filles de 0-10 ans}

L'examen de la table de survie des filles de 0-10 ans révolus de la zone expérimentale révèle une courbe qui se maintient à l'étude finale aux premiers âges pendant 6 ans avant d'amorcer une descente (graphique 15). A l'étude de base, cette courbe amorçait sa descente déjà à l'âge de 3 ans. Ce décalage des deux générations dans cette zone témoigne d'une diminution de l'effectif cumulé des filles excisées. Un écart qui se maintient jusqu'à l'âge de 10 ans, âge auquel la quasi-totalité des filles est excisée. S'il est vrai qu'à l'âge de 6 ans la fille n'a pas encore échappé à l'excision, cela manifeste une régression drastique du nombre de filles qui seront excisées, d'autant plus que l'excision se fait de plus en plus à un âge très jeune. En zone de comparaison, il est difficile de tirer des conclusions fiables compte tenu de l'allure des deux courbes. En effet, on note à l'étude de base une amorce de la courbe à l'âge de 6 ans. A l'étude finale, on assiste à l'entrée en excision des filles dès l'âge de 1 an. Est-ce là le signe d'une certaine recrudescence de la pratique de l'excision dans cette zone ?

\section{Graphique 15 : Proportion cumulée des filles non excisées}

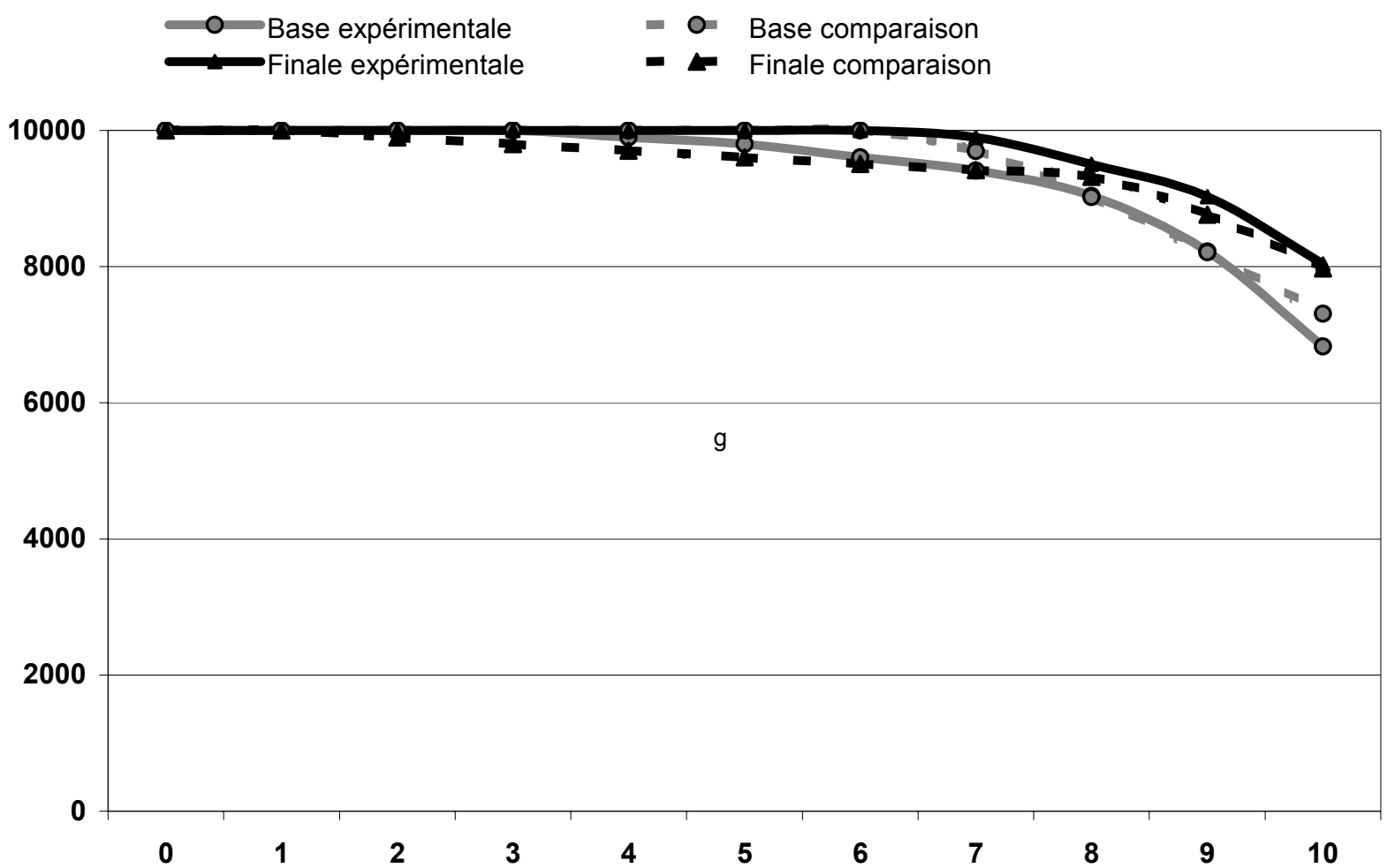

Dans le contexte burkinabé marqué par la loi de 1996, l'effet de la loi n'est pas négligeable. Cependant, la faiblesse des effectifs et la limitation des données ne permettent pas à ce state de mesurer avec précision l'effet réel de la loi et/ou du programme sur la baisse de la pratique et sur ces déclarations, de même que l'effet possible d'un processus global de changement social. 


\section{Diffusion de l'information sur l'excision}

\section{Partage de l'information sur l'excision}

Les différentes campagnes de sensibilisation à travers les médias ainsi que les activités des organisations et associations de lutte contre l'excision ont permis aux femmes et hommes de bénéficier d'information sur l'excision. Cela explique pourquoi en zone expérimentale comme en zone de comparaison, beaucoup d'hommes et de femmes ont déjà reçu des informations sur l'excision. En effet, si au départ 56\% des femmes et $67 \%$ des hommes avaient reçu des informations sur l'excision, ils représentent à l'évaluation finale $99 \%$ des femmes participantes (contre 78\% chez les non participantes) et 100\% des hommes participants (contre $91 \%$ chez les non participants). Au cours des deux dernières années, aucune évolution significative n'est observée dans la zone de comparaison, au contraire de la zone expérimentale. On note dans la zone d'intervention que ceux et celles qui affirment avoir bénéficié d'informations sont significativement plus important qu'à l'étude de base, aussi bien les participants que les non participants. Durant cette période, en plus des classes d'éducation, les habitants de ces villages ont assisté à des saynètes, des représentations, des discussions sur la question de l'excision et à la cérémonie de déclaration publique. Autant de présence et de situations qui permettent de maintenir un niveau d'information et de sensibilisation de la zone.

\section{Graphique 16 : Discute de l'excision avec les autres}

\section{Zone expérimentale}

\section{Zone de comparaison}

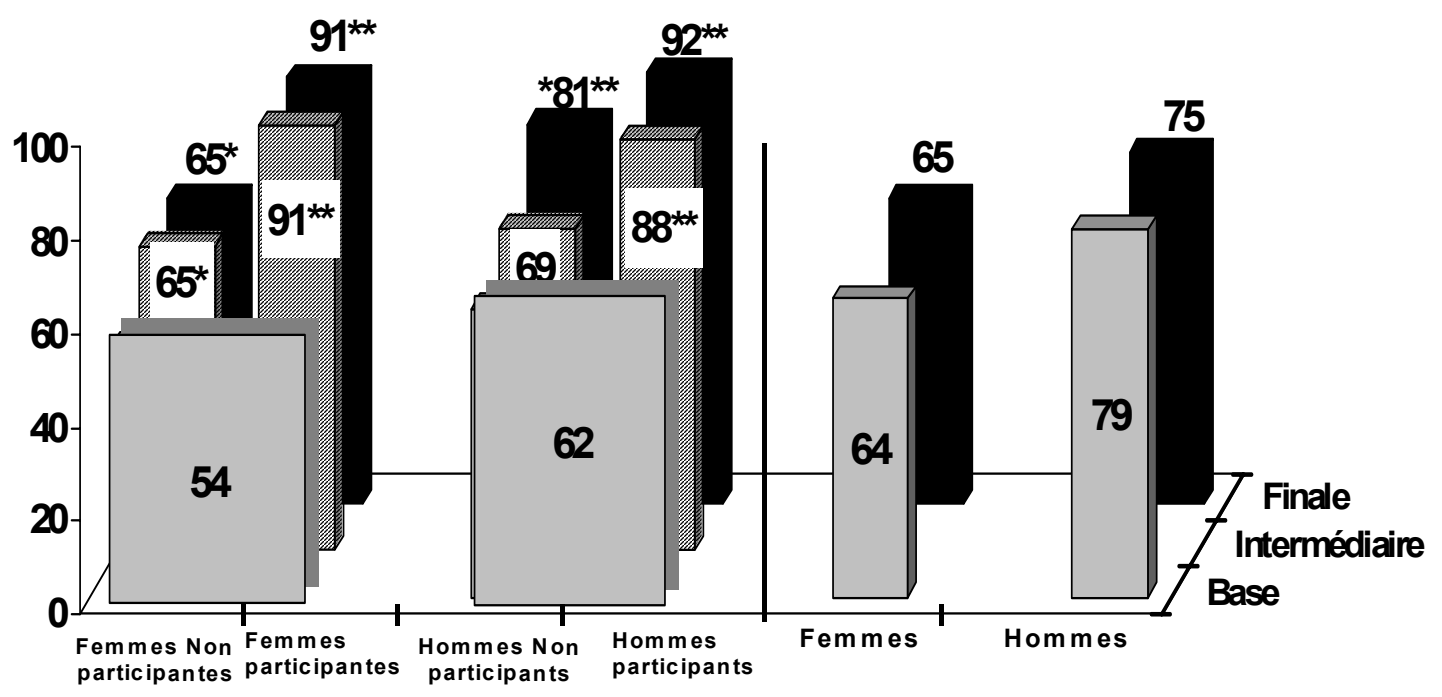

Base 
Aussi, c'est uniquement dans la zone expérimentale que les femmes et les hommes discutent beaucoup de l'excision $(\mathrm{p}<.05)$ (graphique 16). Dans la zone de comparaison, il n'y a pas d'évolution. On note que c'est surtout les participants qui discutent de plus en plus de l'excision.

\section{Graphique 17 : Connaissance de la loi contre l'excision}

\section{Zone expérimentale}

\section{Zone de comparaison}

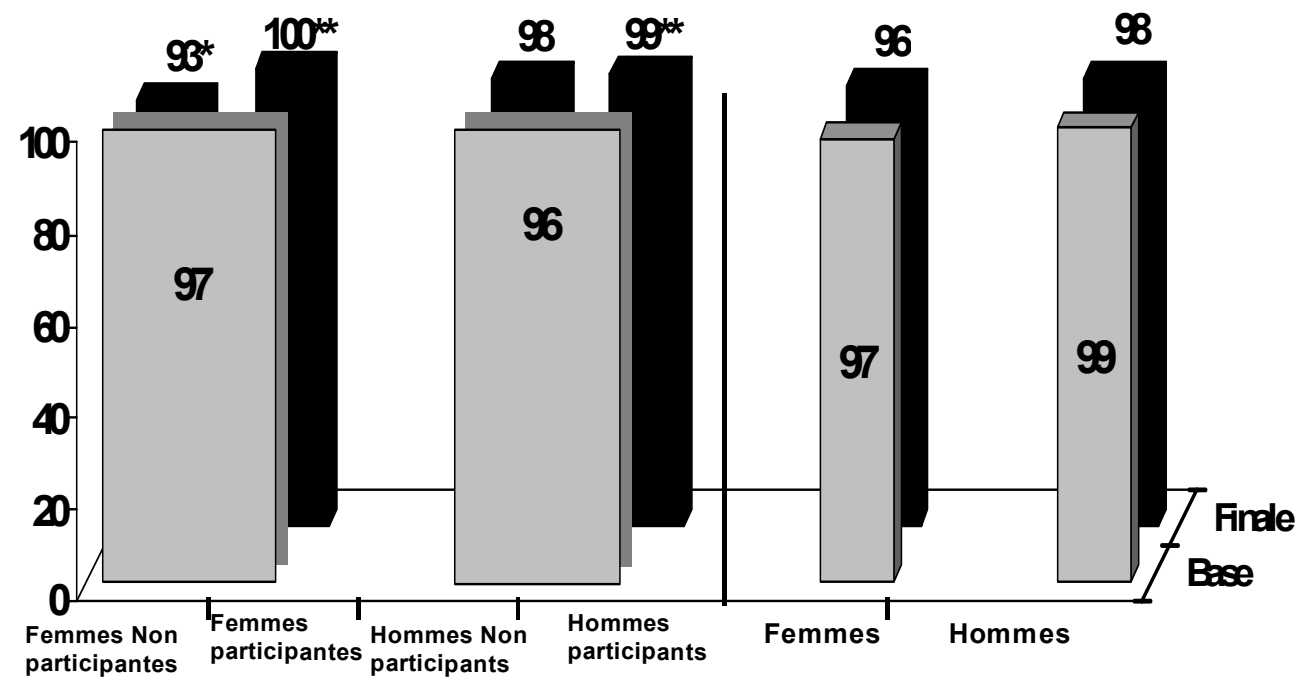

$\square$ Base

DFinkle

Dans leur grande majorité (plus de 90\%), hommes et femmes des deux zones savent qu'une loi interdit la pratique de l'excision (graphique 17), sous peine de sanctions (amendes et emprisonnement). Cette connaissance a augmenté avec le programme $(\mathrm{p}<.01)$. Parmi les femmes non participantes, on assiste cependant à une baisse sensible $(p<.05)$, tandis qu'aucune différence significative n'est observée chez les hommes non participants. En zone de comparaison, il n'y a pas d'évolution notable entre les deux périodes de collecte. ${ }^{1}$

\section{La déclaration publique}

Après la mise en œuvre du programme d'éducation dans les villages, les populations de la zone expérimentale avaient affiché une volonté de mobilisation sociale contre la pratique de l'excision. Cette volonté avait déjà été observée au cours de la collecte des données de l'étude intermédiaire.

En effet, plus de $60 \%$ des personnes interrogées (dans la zone expérimentale) juste après la mise en œuvre du programme ont reconnu qu'il y a eu plus de discussion sur l'excision depuis la mise en œuvre du programme tandis que près de 3 femmes sur 5 et plus de $80 \%$ des

\footnotetext{
${ }^{1}$ La question n'a pas été posée de la même manière à l'étude intermédiaire, ne permettant pas ainsi de faire des comparaisons.
} 
hommes répondent que les femmes du village ont envie de revendiquer l'arrêt de la pratique (tableau 17). A l'évaluation finale, on constate une évolution significative chez toutes les femmes (96\% des participantes contre $77 \%$ des non participantes), contre $60 \%$ des femmes et $66 \%$ des hommes en zone de comparaison. Chez les hommes de la zone expérimentale, il n'y a pas d'évolution significative.

Quant à l'accord des hommes pour la revendication de l'arrêt de la pratique, l'étude intermédiaire a montré que le groupe des participants était plus important à répondre par l'affirmative contre un peu plus de une femme non participante sur deux et 3/4 des hommes non participants (tableau 17). A l'évaluation finale, on note une évolution significative chez l'ensemble des femmes tandis que chez les hommes, c'est chez les participants que l'on voit une évolution significative. En zone de comparaison, les proportions sont restées presque les mêmes entre l'idée de la revendication des femmes et l'accord des hommes.

L'environnement semble être stable, sans idées de revendication, de manifestation en matière de lutte contre la pratique.

\section{Tableau 17 : Mobilisation sociale}

\begin{tabular}{|c|c|c|c|c|c|c|c|}
\hline \multirow{3}{*}{ Indicateurs } & \multicolumn{5}{|c|}{ Zone expérimentale } & \multicolumn{2}{|c|}{$\begin{array}{c}\text { Zone de } \\
\text { comparaison }\end{array}$} \\
\hline & \multirow[b]{2}{*}{ Base } & \multicolumn{2}{|c|}{ Intermédiaire } & \multicolumn{2}{|c|}{ Final } & \multirow[b]{2}{*}{ Base } & \multirow[b]{2}{*}{ Final } \\
\hline & & Participants & $\begin{array}{c}\text { Non } \\
\text { participants }\end{array}$ & Participants & $\begin{array}{c}\text { Non } \\
\text { participants }\end{array}$ & & \\
\hline FEMMES (N) & 662 & 626 & 163 & 578 & 167 & 226 & 228 \\
\hline Les femmes ont envie de revendiq. l'arrêt de l'excision & - & 88 & 59 & $* * 96$ & **77 & - & 60 \\
\hline Les hommes d'accord pour la revendication des femmes & - & 86 & 56 & $* * 95$ & $* * 74$ & - & 56 \\
\hline Je suis prête à débattre publiquement de l'excision & 24 & $71 * *$ & 27 & $* * 84 * *$ & $* * 47 * *$ & 33 & $48 * *$ \\
\hline HOMMES (N) & 697 & 465 & 193 & 448 & 169 & 227 & 229 \\
\hline Les femmes ont envie de revendiq. l'arrêt de l'excision & - & 94 & 87 & 94 & 82 & - & 66 \\
\hline Les hommes d'accord pour la revendication des femmes & - & 83 & 76 & $* * 93$ & 81 & - & 63 \\
\hline Je suis prêt à débattre publiquement de l'excision & 49 & $84 * *$ & $59 *$ & $* * 91 * *$ & $64 * *$ & 61 & 65 \\
\hline
\end{tabular}

Un quart des femmes et la moitié des hommes avaient manifesté leur intérêt à débattre publiquement de l'excision lors de l'étude de base. A l'étude intermédiaire, la proportion des femmes participantes et des hommes a significativement augmenté. Cette tendance se maintient à l'évaluation finale. En zone de comparaison, c'est uniquement parmi les femmes qu'il y a une évolution sensible $(\mathrm{p}<.01)$, puisqu'elles représentent à l'évaluation finale près de la moitié. De part et d'autre, les femmes semblent avoir de plus en plus la volonté de parler ouvertement et en public de la pratique de l'excision.

\section{L'organisation de la déclaration publique}

L'idée d'organiser une déclaration publique est partie du voyage d'étude que des représentants des communautés et de Mwangaza ont fait au Sénégal. En effet au mois de novembre 2000, ces représentants ont assisté à la déclaration publique de Aéré Lao, puis à celle de Karcia en juin 2002. Ceci leur a permis de s'imprégner du processus organisationnel, d'animation d'une cérémonie de déclaration publique et de comprendre ce processus de mobilisation sociale.

De retour au Burkina, des réunions villageoises et inter villageoises ont permis aux membres de la délégation de partager l'expérience du Sénégal. A l'issue de ces réunions qui ont marqué la décision de prendre un engagement dans la lutte contre l'excision, un atelier a regroupé des représentants des 23 villages pour réfléchir sur la nécessité d'officialiser cette 
décision. Il a été retenu à cet atelier qu'une femme prendrait la parole pour présenter la décision collective des deux départements concernés. Elle serait suivie par un représentant des responsables coutumiers qui posera un geste symbolique d'engagement et de soutien à la décision.

Cette cérémonie de déclaration publique d'abandon de l'excision a eu lieu le 3 mai 2003. Elle a connu la participation des 34 autres villages de Béré et Bindé. A l'issue de cette journée de déclaration, une série de questions a été adressée aux populations de la zone d'intervention. Ces questions avaient pour but de mesurer le degré d'implication des populations mais également de mesurer leur perception sur la durabilité d'une telle décision.

\section{Graphique 18 : Informations sur la cérémonie de déclaration publique}

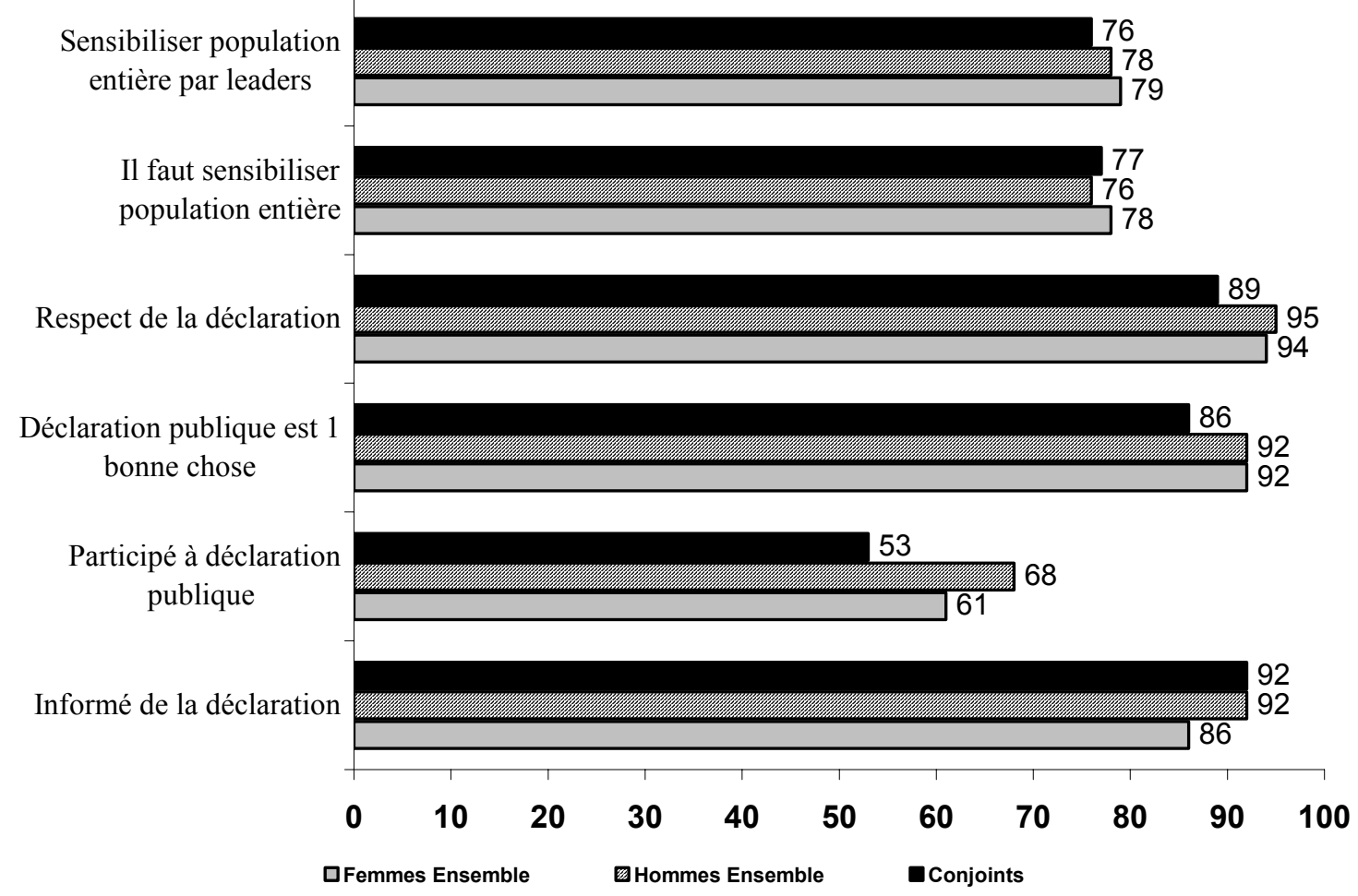

Au graphique 18, on voit que plus de trois quarts des femmes et des hommes ont été informés de la tenue de la cérémonie de déclaration publique et plus de la moitié y ont participé. Hommes comme femmes de la zone expérimentale estiment que cette cérémonie est une bonne chose et restent convaincus que cette décision prise de ne plus exciser les filles sera respectée par tous et qu'aucune fille ne sera plus excisée dans la zone. Au cours d'entretien informel, une femme nous a confié ceci : "Oui, la décision de ne plus pratiquer l'excision sera respectée. Parce que les gens passeront maintenant le temps à s'épier les uns les autres afin de connaître le premier qui ne respecterait pas sa parole ${ }^{\prime \prime}$. Un tel sentiment de peur du regard de l'autre pourrait ainsi induire un respect strict de la décision prise. Les femmes comme les hommes estiment qu'il faut néanmoins poursuivre la sensibilisation de la population entière, notamment une sensibilisation assurée par les leaders politiques et religieux.

\footnotetext{
${ }^{1}$ Déclaration d'une femme au cours d'un entretien informel dans le village de Béré.
} 
Il y a un engagement certain des participants au programme à la déclaration publique (66\% des femmes et $74 \%$ des hommes). De plus, ils sont relativement nombreux à soutenir que la décision prise sera respectée et que les filles ne seront plus jamais excisées. La plus faible proportion des non participants ayant participé à la déclaration publique $(27 \%$ des femmes et $53 \%$ des hommes) témoigne de la nécessité de renforcer les acquis pour que les bonnes intentions s'inscrivent dans un processus de changement social. Il faut continuer à soutenir cette initiative par des actions diverses comprenant à la fois des séances de sensibilisation mais également l'organisation de manifestations à caractère public autour du thème de la pratique de l'excision.

\section{Le besoin d'approfondissement des connaissances}

L'intérêt que suscite le programme dans la zone d'intervention s'est traduit par la demande adressée par d'autres villages de la zone pour y participer. Un autre intérêt s'est exprimé dans les villages d'intervention où selon les participants ( $98 \%$ de femmes et d'hommes), les filleuls et toutes les personnes avec qui ils auraient échangé sur les modules expriment le besoin d'approfondir ces connaissances acquises auprès des participants. Ils souhaiteraient donc que le programme soit réédité et élargi à d'autres localités. 


\section{Conclusion}

On a pu noter un engouement manifeste à suivre le programme chez les femmes. La proportion des participants ayant suivi tous les modules est élevée $(63 \%$ des femmes et $60 \%$ des hommes), avec un niveau de participation élevé chez les hommes âgés. Parmi les modules enseignés, l'hygiène et la prévention des maladies et celui sur la santé de la femme sont ceux qui ont le plus intéressé les participants. Le manque de mesures

d'accompagnement, l'absence de classes formelles et le changement de facilitateurs sont les principales insuffisances du programme constatées par les participants.

\section{Un niveau de connaissance plus élevé}

Dans l'ensemble, hommes et femmes participants au programme ont amélioré leur niveau de connaissance sur les droits humains, le planning familial, les consultations prénatales, les signes de danger de la grossesse, l'excision, les IST/VIH/SIDA, les différentes formes de violences contre les femmes et les formes de discrimination contre les femmes. Pour un certain nombre d'indicateurs, la zone de comparaison a aussi évoluée positivement.

Cependant pour les indicateurs sur les droits humains, la PF, les signes de danger de la grossesse, les IST/VIH/SIDA, les dangers de l'excision, la zone d'intervention montre une plus forte évolution que la zone de comparaison. Les femmes et les hommes participants dans la zone d'intervention montrent une plus forte progression que les femmes et les hommes non participants. Pour les indicateurs sur les moyens de prévention des IST/VIH/SIDA, les dangers de l'excision, chez les femmes non participantes aucune évolution n'a été notée.

En matière de droits humains, le niveau de connaissance des droits a significativement évolué en zone expérimentale et reste attribuable au programme, notamment chez les femmes. Celui des hommes n'est pas seulement attribuable au programme, car montrant le même niveau d'évolution que la zone de comparaison. Pour ce qui concerne les formes de discrimination, hommes et femmes participants sont significativement plus nombreux qu'à l'étude de base à soutenir qu'il existe dans la communauté des pratiques qui avantagent beaucoup plus les hommes que les femmes. Bien que les proportions soient très élevées chez les femmes du programme, cette évolution n'est pas attribuable au programme, puisqu'on enregistre une évolution aussi significative en zone de comparaison. Pourtant, les différentes formes de violences sont de plus en plus reconnues en zone expérimentale, bien que ce niveau de reconnaissance ne soit seulement attribuable au programme que pour le groupe des femmes.

En matière de planification familiale, les participants au programme ont nettement augmenté leur niveau de connaissance des méthodes modernes de contraception, comparativement aux femmes et hommes non participants et comparativement à la zone de comparaison. Quant aux informations concernant la santé des femmes, les femmes du programme ont substantiellement amélioré leur niveau de connaissance sur le nombre minimum de consultations prénatales, contrairement aux hommes qui conservent encore le même niveau. Pour ce qui est des signes de danger de la grossesse, le programme à lui seul a permis d'améliorer le niveau de connaissance des hommes participants. Les effets néfastes de l'excision sont surtout connus par les femmes, contrairement aux hommes pour lesquels le niveau n'a pas évolué. Sur les questions liées à l'alimentation, hommes et femmes participants sont relativement plus nombreux à soutenir qu'une bonne habitude alimentaire contribue à lutter contre la diarrhée. 
Sur les IST/VIH/SIDA, le programme a fortement permis d'augmenter les connaissances sur la notion de séropositivité, surtout parmi les participants au programme. Quant aux moyens de prévention des IST, seuls les participants ont amélioré leur connaissance.

\section{Des attitudes plus positives}

Si les femmes de la zone expérimentale, surtout les participantes vivent de moins en moins les violences dans leurs foyers, il y a quand même lieu de noter que la proportion des hommes qui approuvent les violences est encore élevée. Cependant, on a pu noter que des changements sont intervenus depuis la mise en œuvre du programme, notamment la diminution des disputes et une meilleure écoute des femmes, alors qu'en zone de comparaison l'approbation de ces violences a connu une augmentation sensible aussi bien chez les femmes que chez les hommes. A propos de la planification familiale, c'est surtout les participants qui soutiennent que la décision d'utilisation de la contraception doit être prise conjointement par les deux époux. Ce sont également les participants qui ont significativement évolué dans leur désir d'utilisation des méthodes contraceptives modernes dans l'avenir. Aucune évolution n'est remarquée pour les non participants et les populations des zones de comparaison.

L'idée que la discrimination des hommes à l'égard des femmes peut changer est grandement partagée par les femmes, mais également par les hommes, malgré que ces derniers demeurent encore conservateurs. Cette nouvelle conviction des femmes est essentiellement attribuable au programme d'intervention.

Concernant l'excision, il y a plus d'hommes et de femmes de la zone expérimentale, à déclarer que la pratique n'est plus nécessaire. Cette évolution de l'opinion sur la nécessité de cette pratique est en grande partie attribuable au programme chez les femmes mais pas pour les hommes. Les femmes participantes sont plus nombreuses à regretter l'excision de leurs filles et ne pas approuver la pratique. Presque toutes soutiennent que leurs filles (non encore excisées) ne le seront pas. En zone de comparaison par contre, la proportion des hommes désirant exciser les filles a connu une augmentation significative.

\section{Des partenaires plus ouverts}

La mise en œuvre du programme a permis à un dixième des participants (hommes et femmes) de mieux communiquer, notamment dans le couple. Au-delà de l'importance de l'agent de santé dans l'orientation médicale de la femme, aussi bien les femmes que les hommes, notamment les participants, soutiennent que leur(s) partenaire(s) approuve(nt) l'utilisation de la contraception moderne. Une attitude qui reste toujours à améliorer chez les conjoints des femmes non participantes et dans la zone de comparaison.

\section{Une pratique de l'excision faible mais en baisse}

Malgré une prévalence réelle de l'excision des filles qui se maintient autour de 43\%, la proportion des filles de moins de 5 ans excisées est en régression dans la zone. Les données actuelles ne permettent pas de mesurer avec précision l'effet réel de la contribution du programme d'intervention.

\section{Une mobilisation sociale importante}

Les différentes activités menées dans la zone expérimentale durant la mise en œuvre du programme ont permis aux habitants de ces villages de bénéficier d'informations sur l'excision. C'est pourquoi on a pu noter que dans cette zone il y a eu plus de discussion sur ce sujet. La proportion de femmes et d'hommes de la zone d'intervention qui affirment être prêt 
pour discuter en public de l'excision a sensiblement augmenté. En zone de comparaison, ce sont les femmes qui ont connu une telle évolution, mais avec un degré moins élevé. Toutes ces informations et discussions ont été renforcées depuis la cérémonie de déclaration publique qui a regroupé aussi bien des villages d'intervention que des villages situés en dehors de la zone d'intervention. Hommes et femmes de la zone expérimentale demeurent convaincus que cette déclaration sera respectée. Mais pour cela, il y a quand même lieu de sensibiliser davantage les leaders de la communauté afin de toucher toute la population entière des villages.

Malgré les niveaux déclarés très faibles d'excision, la déclaration publique a été jugée nécessaire par les représentants des communautés. En effet, elle permet à toute une communauté d'affirmer une volonté de ne plus pratiquer l'excision. De décision individuelle, souvent guidée par la peur des répressions, on passe à une décision de groupe. Le support social du groupe devrait permettre de maintenir cette décision.

La faiblesse des évolutions chez les non-participants vivant dans les villages d'intervention témoigne du faible fonctionnement du système de parrainage mis en place dans le cadre du programme et qui avait pour rôle de diffuser les informations à travers la communauté. Une situation confortée par les demandes de participation et de plus d'informations adressées par ces non participants dans les villages du programme, mais également par la demande de participation des autres villages en marge de la zone d'intervention. Autant de besoins qui restent à combler. 


\section{Références}

Congo Zakari, Diop Nafissatou, Ouoba Djingri (2001). Evaluation d'un programme à base communautaire, rapport de base, Population Council Dakar 43 pages.

Congo Zakari, Diop Nafissatou, Ouoba Djingri (2002). Evaluation d'un programme à base communautaire, rapport d'évaluation intermédiaire, Ouagadougou.

Comité national de lutte contre la pratique de l'excision (CNLPE). 1999. Plan d'action de lutte contre la pratique de l'excision (1999-2003), Ouagadougou, 39 pages.

Dera Lassané, Ilboudo François, Kabore Idrissa, Ouattara Adama, Ye Aminata (1997). Enquête Nationale sur l'Excision au Burkina Faso, INSD, Ouaga, 62 pages.

Konaté Désiré Lohé, Tinga Sinaré, et Michka Seroussi. 1993. Enquête Démographique et de Santé, Burkina Faso, 1993. Calverton, Maryland: Institut National de la Statistique et de la Démographie et Macro International, Inc.

Laboratoire de Santé Communautaire (1998). Evaluation de la prévalence, de la typologie et des complications liées à l'excision chez les patients fréquentant les formations sanitaires du Bazèga, Série documentaire $\mathrm{N}^{\circ} 21$, Ouagadougou, novembre, 14 pages.

Laboratoire de Santé Communautaire (1998). Etude participative pour l'identification des stratégies communautaires de lutte contre la pratique de l'excision dans le Bazèga, Série documentaire $\mathrm{N}^{\circ} 22$, Ouagadougou, décembre, 30 pages.

Ministère de la Santé (2000). Protocoles de santé de la reproduction, santé de la femme, de l'homme, des jeunes, Burkina Faso, septembre, 183 pages.

Ministère de la Santé (2000). Protocoles des composantes d'appui en santé de la reproduction, Burkina Faso, septembre, 27 pages.

Ministère de la Santé (2000). Politique et normes de services de santé de la reproduction, Burkina Faso, septembre, 76 pages.

Ministère de la Santé (2000). Composantes communes en santé de la reproduction, Burkina Faso, septembre, 185 pages.

Mwangaza Action, Population Council, TOSTAN (2003). Comment 23 villages abandonnent la pratique de l'excision. Documentation de la mise en œuvre du programme TOSTAN au Burkina Faso, Avril, 45 pages.

Njeri Chege Jane, Askew Ian, Liku Jennifer (2001). An assessment of the Alternative Rites Approach for Encouraging Abandonment of Female Genital Mutilation in Kenya, FRONTIERS, June, 48 pages.

Ouedraogo Diénéba, Kaboré Yimian, Sanon Edème (2000). Etude de base sur la pratique de l'excision dans cinq provinces du Burkina Faso, GTZ, 92 pages. 
Sangli Gabriel (2001). Enquête de prévalence sur la pratique de l'excision et l'impact des actions menées dans sept provinces du Burkina, UNICEF-CNLPE, Avril, 57 pages

Tamini Ida, Diop Nafissatou (2002). Evaluation d'un programme à base communautaire, le point de vue des facilitateurs, rapport d'étude, 13 pages.

Tamini Ida, Diop Nafissatou, Ouoba Djingri (2001). Evaluation d'un programme à base communautaire. Etude qualitative de base. 25 pages

TOSTAN (1999). Breakthrough in Senegal : Ending Female Genital Cutting, Dakar, Senegal.

Toubia Nahid and Susan IZETT (1999). Learning About Social Change. A research and evaluation guidebook using female circumcision as a case study. New York : Rainbo

UERD (Unité D’Enseignement et de Recherche en Démographie), Direction de la Santé de la Famille, et Population Council (1997). Rapport Provisoire de l'Enquête Quantitative de Base. Ouagadougou, Burkina Faso: UERD. 


\begin{tabular}{|c|c|c|c|c|c|c|c|}
\hline \multicolumn{8}{|c|}{ 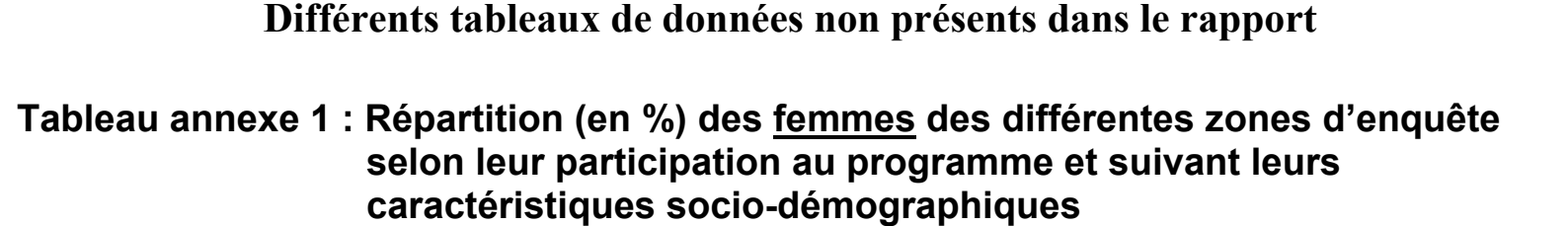 } \\
\hline \multirow{3}{*}{ Indicateurs } & \multicolumn{5}{|c|}{ Zone expérimentale } & \multicolumn{2}{|c|}{ Zone de comparaison } \\
\hline & \multirow[b]{2}{*}{ Base } & \multicolumn{2}{|c|}{ Intermédiaire } & \multicolumn{2}{|c|}{ Final } & \multirow[b]{2}{*}{ Base } & \multirow[b]{2}{*}{ Final } \\
\hline & & Participantes & $\begin{array}{c}\text { Non } \\
\text { participantes }\end{array}$ & Participantes & $\begin{array}{c}\text { Non } \\
\text { participantes }\end{array}$ & & \\
\hline \multicolumn{8}{|l|}{ Age } \\
\hline Moins de 20 ans & 12 & 8 & 3 & 5 & 8 & 1 & 4 \\
\hline $20-29$ ans & 39 & 34 & 35 & 36 & 25 & 32 & 29 \\
\hline $30-39$ ans & 28 & 31 & 30 & 29 & 35 & 35 & 33 \\
\hline $40-49$ ans & 14 & 18 & 15 & 20 & 23 & 27 & 19 \\
\hline 50 et plus & 7 & 9 & 17 & 10 & 9 & 5 & 15 \\
\hline Age moyen & 31 & $33 *$ & $35 * *$ & $34 * *$ & $34 * *$ & 34 & 36 \\
\hline Age médian & 29 & 31 & 32 & 32 & 34 & 33 & 35 \\
\hline \multicolumn{8}{|c|}{ Niveau d'Instruction } \\
\hline Sans instruction & 90 & 90 & $97 * *$ & 90 & $95^{*}$ & 92 & 94 \\
\hline Primaire & 9 & 9 & 3 & 9 & 5 & 7 & 5 \\
\hline Second et plus & 1 & 1 & 0 & 1 & 0 & 1 & 1 \\
\hline \multicolumn{8}{|l|}{ Religion } \\
\hline Musulmans & 72 & 71 & 68 & 71 & 71 & 20 & 17 \\
\hline Catholiques & 23 & 22 & 25 & 22 & 22 & 52 & 49 \\
\hline Protestants & 4 & 5 & 3 & 5 & 2 & 7 & 5 \\
\hline Traditionnels & 1 & 2 & 4 & 2 & 5 & 21 & 29 \\
\hline \multicolumn{8}{|l|}{ Etat Matrimonial } \\
\hline Mariée monog. & 35 & 32 & $49^{* *}$ & $29 *$ & $46^{* *}$ & 56 & $47 *$ \\
\hline Mariée polyg. & 60 & 60 & 51 & 62 & 54 & 44 & 53 \\
\hline Célibataire & 4 & 4 & - & 6 & - & - & - \\
\hline Veufs/Divorcés & 1 & 4 & - & 3 & - & - & - \\
\hline \multicolumn{8}{|l|}{ Ethnie } \\
\hline Mossi & 99 & 100 & 100 & 99 & 99 & 99 & 100 \\
\hline Autres ethnies & 1 & 0 & 0 & 1 & 1 & 1 & 0 \\
\hline \multicolumn{8}{|l|}{ Alphabétisation } \\
\hline Oui & 27 & $36^{* *}$ & $9 * *$ & $36^{* *}$ & $11^{* *}$ & 23 & 28 \\
\hline Non & 73 & 64 & 91 & 64 & 89 & 77 & 72 \\
\hline \multicolumn{8}{|c|}{ Aut formation antér. } \\
\hline Oui & 5 & $12^{* *}$ & $1 *$ & $87 * *$ & 2 & 11 & 15 \\
\hline Non & 95 & 88 & 99 & 13 & 98 & 89 & 85 \\
\hline Effectifs & 662 & 626 & 163 & 578 & 167 & 226 & 228 \\
\hline
\end{tabular}




\section{Tableau annexe 2 : Répartition (en \%) des hommes des différentes zones d'enquête selon leur participation au programme et suivant leurs caractéristiques socio-démographiques}

\begin{tabular}{|c|c|c|c|c|c|c|c|}
\hline \multirow{3}{*}{ Indicateurs } & \multicolumn{5}{|c|}{ Z Zone expérimentale } & \multicolumn{2}{|c|}{ Zone de comparaison } \\
\hline & \multirow[b]{2}{*}{ Base } & \multicolumn{2}{|c|}{ Intermédiaire } & \multicolumn{2}{|c|}{ Final } & \multirow[b]{2}{*}{ Base } & \multirow[b]{2}{*}{ Final } \\
\hline & & Participants & $\begin{array}{c}\text { Non } \\
\text { participants } \\
\end{array}$ & Participants & $\begin{array}{c}\text { Non } \\
\text { participants } \\
\end{array}$ & & \\
\hline \multicolumn{8}{|l|}{ Age } \\
\hline Moins de 20 ans & 6 & 9 & 2 & 4 & 0 & 0 & 0 \\
\hline $20-29$ ans & 22 & 24 & 12 & 25 & 14 & 8 & 10 \\
\hline $30-39$ ans & 27 & 29 & 20 & 28 & 23 & 26 & 18 \\
\hline $40-49$ ans & 23 & 19 & 21 & 22 & 23 & 29 & 21 \\
\hline 50 et plus & 22 & 19 & 45 & 21 & 40 & 37 & 51 \\
\hline Age moyen & 39 & $36^{* *}$ & $47 * *$ & 38 & $45^{* *}$ & 45 & $49 * *$ \\
\hline Age médian & 37 & 35 & 47 & 37 & 43 & 45 & 50 \\
\hline \multicolumn{8}{|l|}{ Niveau d'Instruction } \\
\hline Sans instruction & 86 & 81 & 90 & 79 & 87 & 91 & 88 \\
\hline Primaire & 12 & 18 & 9 & 19 & 11 & 8 & 12 \\
\hline Second et plus & 2 & 1 & 1 & 2 & 2 & 1 & 0 \\
\hline \multicolumn{8}{|l|}{ Religion } \\
\hline Musulmans & 69 & 70 & 72 & 72 & 72 & 20 & 20 \\
\hline Catholiques & 20 & 23 & 21 & 20 & 20 & 44 & 38 \\
\hline Protestants & 5 & 6 & 2 & 5 & 2 & 3 & 4 \\
\hline Traditionnels & 6 & 1 & 5 & 3 & 6 & 33 & 38 \\
\hline \multicolumn{8}{|l|}{ Etat Matrimonial } \\
\hline Mariée monog. & 43 & 47 & 46 & 48 & 50 & 53 & 48 \\
\hline Mariée polyg. & 47 & 37 & 49 & 39 & 50 & 47 & 52 \\
\hline Célibataire & 10 & 15 & 4 & 2 & - & - & - \\
\hline Veufs/Divorcés & 0 & 1 & 1 & 11 & - & - & - \\
\hline \multicolumn{8}{|l|}{ Ethnie } \\
\hline Mossi & 100 & 100 & 100 & 100 & 99 & 98 & 100 \\
\hline Autres ethnies & 0 & 0 & 0 & 0 & 1 & 2 & 0 \\
\hline \multicolumn{8}{|l|}{ Alphabétisation } \\
\hline Oui & 25 & $44 * *$ & $18 *$ & $48 * *$ & $15 * *$ & 33 & 31 \\
\hline Non & 75 & 56 & 82 & 52 & 85 & 67 & 69 \\
\hline $\begin{array}{l}\text { Aut formation antér. } \\
\text { Oui }\end{array}$ & 12 & $22^{* *}$ & 11 & $89 * *$ & $5^{* *}$ & 33 & $24 *$ \\
\hline Non & 88 & 78 & 89 & 11 & 95 & 66 & 76 \\
\hline Effectifs & 697 & 465 & 193 & 448 & 169 & 227 & 229 \\
\hline
\end{tabular}


Tableau annexe 3 : Caractéristiques socio-démographiques des conjoints de femmes participantes au programme

\begin{tabular}{|c|c|c|c|}
\hline \multirow{2}{*}{ Caractéristiques } & \multicolumn{3}{|c|}{ Zone Expérimentale } \\
\hline & Base & Intermédiaire & Final \\
\hline \multicolumn{4}{|l|}{ Age } \\
\hline Moins de 20 ans & 1 & 0 & 0 \\
\hline $20-29$ ans & 14 & 12 & 12 \\
\hline $30-39$ ans & 19 & 21 & 21 \\
\hline $40-49$ ans & 23 & 21 & 20 \\
\hline 50 et plus & 43 & 46 & 47 \\
\hline Age moyen & 46 & 48 & 48 \\
\hline Age médian & 47 & 48 & 48 \\
\hline \multicolumn{4}{|l|}{ Niveau d'Instruction } \\
\hline Sans instruction & 88 & 89 & 88 \\
\hline Primaire & 10 & 11 & 10 \\
\hline Second et plus & 2 & 0 & 2 \\
\hline \multicolumn{4}{|l|}{ Religion } \\
\hline Musulmans & 73 & 68 & 69 \\
\hline Catholiques & 14 & 22 & 17 \\
\hline Protestants & 4 & 3 & 3 \\
\hline Traditionnels & 9 & 7 & 11 \\
\hline \multicolumn{4}{|l|}{ Etat Matrimonial } \\
\hline Mariée monogame & 36 & 40 & 40 \\
\hline Mariée polygame & 64 & 60 & 60 \\
\hline \multicolumn{4}{|l|}{ Ethnie } \\
\hline Mossi & 100 & 100 & 99 \\
\hline Autres ethnies & 0 & 0 & 1 \\
\hline \multicolumn{4}{|l|}{ Alphabétisation } \\
\hline Oui & 18 & 22 & 19 \\
\hline Non & 82 & 78 & 81 \\
\hline \multicolumn{4}{|l|}{ Aut formation antér. } \\
\hline Oui & 12 & 12 & $6 *$ \\
\hline Non & 88 & 88 & 94 \\
\hline Effectifs & 315 & 295 & 281 \\
\hline
\end{tabular}


Tableau annexe 4 : Proportion cumulée des filles non excisées

\begin{tabular}{ccccc}
\hline \multirow{2}{*}{ Ages } & \multicolumn{3}{c}{ Expérimentale } & Zone de comparaison \\
\cline { 2 - 4 } & $\begin{array}{c}\text { Base }(\%) \\
N=556\end{array}$ & $\begin{array}{c}\text { Final }(\%) \\
N=690\end{array}$ & $\begin{array}{c}\text { Base }(\%) \\
N=260\end{array}$ & $\begin{array}{c}\text { Final }(\%) \\
N=217\end{array}$ \\
\cline { 2 - 4 } 0 & 1,000 & 1,000 & 1,000 & 1,000 \\
1 & 1,000 & 1,000 & 1,000 & 0,995 \\
2 & 1,000 & 1,000 & 0,995 \\
3 & 0,995 & 1,000 & 0,995 \\
4 & 0,995 & 1,000 & 1,000 & 0,988 \\
5 & 0,980 & 0,997 & 1,000 & 0,988 \\
6 & 0,976 & 0,993 & 1,000 & 0,988 \\
7 & 0,958 & 0,964 & 0,972 & 0,988 \\
8 & 0,911 & 0,949 & 0,932 & 0,939 \\
9 & 0,831 & 0,886 & 0,913 & 0,911 \\
10 & 0,545 & 0,777 & 0,886 & 0,746 \\
\hline \hline
\end{tabular}

